\title{
Ruthenium-Catalyzed Enantioselective Hydrogenation of Hydrazones
}

Christopher H. Schuster ${ }^{1}$, James F. Dropinski ${ }^{1}$, Michael Shevlin ${ }^{1}$, Hongming $\mathrm{Li}^{1}$, and Song Chen $^{2}$

${ }^{1}$ Department of Process Research, Merck Research Laboratories, Merck \& Co., Inc., Rahway, New Jersey, 07065.

${ }^{2}$ Department of Synthetic Chemistry, Pharmaron Beijing Co., Ltd., No. 6, Tai-He Road, BDA, Beijing, 100176 P.R. China

Supporting Information

Table of Contents

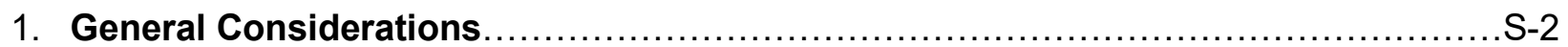

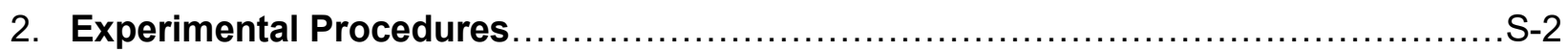

2.1. Representative procedure for hydrazone synthesis $\ldots \ldots \ldots \ldots \ldots \ldots \ldots \ldots \ldots \ldots \ldots . \mathrm{S}-2$

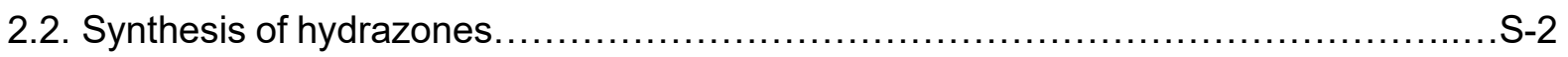

2.3. Representative procedure for hydrazone hydrogenation $\ldots \ldots \ldots \ldots \ldots \ldots \ldots \ldots \ldots \ldots \ldots . \mathrm{S}-7$

2.4. Synthesis and characterization of hydrazine products $\ldots \ldots \ldots \ldots \ldots \ldots \ldots \ldots \ldots \ldots \ldots . \mathrm{S}-8$

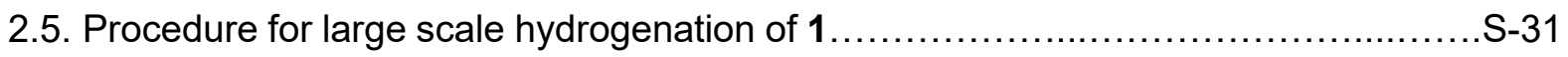

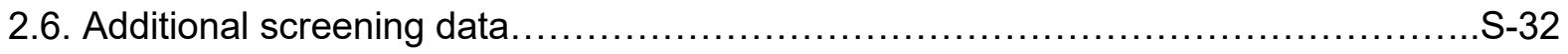

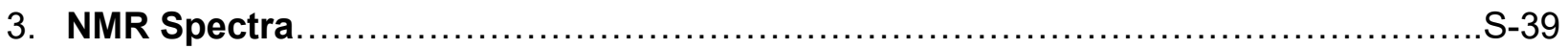

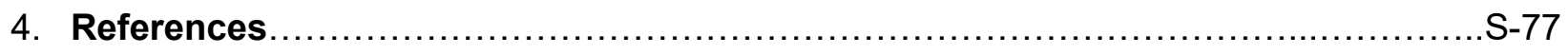




\section{General Considerations}

All air and moisture sensitive manipulations were carried out using standard vacuum line, Schlenk, and cannula techniques or in an inert atmosphere dry box containing an atmosphere of nitrogen. All reagents were purchased from commercial vendors and used as received. ${ }^{1} \mathrm{H}$ NMR spectra were recorded using spectrometers operating at 300 or $400 \mathrm{MHz}$. Chemical shifts are reported in parts per million (ppm) with the solvent resonance as the internal standard (DMSO: $2.50 \mathrm{ppm}$ or $\mathrm{MeOH}: 3.31 \mathrm{ppm})$. Data are reported as follows: chemical shift, integration, multiplicity $(\mathrm{s}=$ singlet, $\mathrm{d}=$ doublet, $\mathrm{t}=$ triplet, $\mathrm{q}=$ quartet, $\mathrm{p}=$ pentet, $\mathrm{m}=$ multiplet, app $=$ apparent), and coupling constants $(\mathrm{Hz}) .{ }^{13} \mathrm{C} \quad\left\{{ }^{1} \mathrm{H}\right\}$ NMR spectra were recorded using spectrometers operating at 75 or $100 \mathrm{MHz}$. Chemical shifts are reported in ppm with the solvent resonance as the internal standard (DMSO- $\mathrm{d}_{6}: 39.52 \mathrm{ppm}$ or MeOD- $\mathrm{d}_{4}: 49.00 \mathrm{ppm}$ ). All racemic products were obtained by reduction of the appropriate hydrazone starting materials with sodium cyanoborohydride. Absolute stereochemistry was assigned by analogy to compound 18, which was directly compared to authentic material derived from commercial (S)-1-phenylethylamine according to the method of Armstrong and co-workers. ${ }^{1}$

\section{Experimental Procedures}

\section{1 - Representative Procedure for Hydrazone Synthesis}<smiles></smiles>

To a round bottom flask equipped with stir bar and flushed with nitrogen was added ketone (1 equiv.) as a solution in dry methanol $(1.0 \mathrm{M})$. A methanol solution of Cbz-hydrazide (1 equiv., $0.66 \mathrm{M}$ ) was then added to give a clear solution which was stirred at room temperature. After 2 hours at room temperature, a solid precipitate was formed. Reaction progress was monitored by UPLC analysis of the reaction mixture. After a total of 3.5 hours at room temperature, starting material was consumed. The resulting reaction mixture was cooled to $0{ }^{\circ} \mathrm{C}$ in an ice bath and the solid was collected on a disposable frit, rinsing with a small amount of cold $\mathrm{MeOH}$. The solid was then dried under vacuum to give the desired hydrazone product. All hydrazone products were formed as the $(E)$ isomers unless otherwise noted.

\section{2 - Synthesis of Hydrazones}

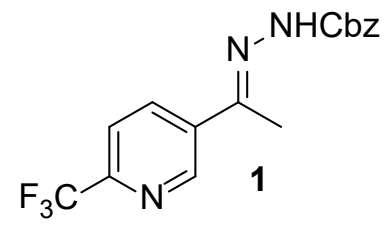

benzyl (E)-2-(1-(6-(trifluoromethyl)pyridin-3-yl)ethylidene)hydrazine -1-carboxylate (compound 1, Table 1). Prepared according to the general procedure utilizing 1-(6-(trifluoromethyl)pyridin-3-yl)ethan-1-one $(1.53 \mathrm{~g}, 8.1 \mathrm{mmol})$ and benzyl hydrazinecarboxylate $(1.34 \mathrm{~g}, 8.1 \mathrm{mmol})$ to give the title product as a white solid $(2.4 \mathrm{~g}, 88 \%) .{ }^{1} \mathrm{H}$ NMR $(300 \mathrm{MHz}$, DMSO-d $\left.\mathrm{d}_{6}\right): \delta 10.60(1 \mathrm{H}, \mathrm{s}), 9.06(1 \mathrm{H}, \mathrm{s}), 8.35(1 \mathrm{H}, \mathrm{d}, J=8.1 \mathrm{~Hz}), 7.94(1 \mathrm{H}, \mathrm{d}, J=8.3 \mathrm{~Hz}), 7.51-$ $7.30(5 \mathrm{H}, \mathrm{m}), 5.23(2 \mathrm{H}, \mathrm{s}), 2.30(3 \mathrm{H}, \mathrm{s}) .{ }^{13} \mathrm{C}$ NMR (100 MHz, DMSO-d 6 ): (2 overlapping signals) 
$\delta 154.0,147.7,145.9\left(\mathrm{q}, J_{C-F}=34.0 \mathrm{~Hz}\right), 137.1,136.5,135.3,128.5,128.1,121.7\left(\mathrm{q}, J_{C-F}=273.8\right.$ $\mathrm{Hz}$ ), $120.4\left(\mathrm{q}, \mathrm{J}_{\mathrm{C}-\mathrm{F}}=2.8 \mathrm{~Hz}\right), 66.3,13.8$. HRMS (ESI) $\mathrm{m} / \mathrm{z}:[\mathrm{M}+\mathrm{H}]^{+}$Calculated for $\mathrm{C}_{16} \mathrm{H}_{15} \mathrm{~N}_{3} \mathrm{O}_{2} \mathrm{~F}_{3}$ 338.1116 ; found 338.1115 .

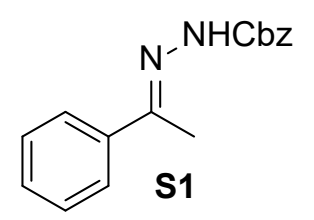

benzyl (E)-2-(1-phenylethylidene)hydrazine-1-carboxylate (S1). Prepared according to the general procedure utilizing acetophenone $(1.0 \mathrm{~g}, 8.4 \mathrm{mmol})$ and benzyl hydrazinecarboxylate $(1.4 \mathrm{~g}, 8.4 \mathrm{mmol})$ to give the title product as a white solid $(2.2 \mathrm{~g}, 98 \%)$. All spectral data are in accordance with the literature. ${ }^{2}$

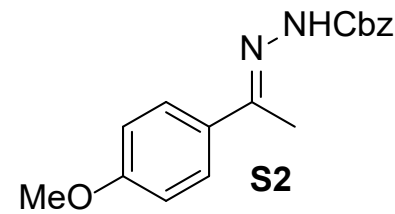

benzyl (E)-2-(1-(4-methoxyphenyl)ethylidene)hydrazine-1-carboxylate (S2). Prepared according to the general procedure utilizing 1-(4methoxyphenyl)ethan-1-one $(1.35 \mathrm{~g}, 9.0 \mathrm{mmol})$ and benzyl hydrazinecarboxylate $(1.5 \mathrm{~g}, 9.0 \mathrm{mmol})$ to give the title product as a white solid (2.5 g, 93\%). ${ }^{1} \mathrm{H}$ NMR (400 MHz, DMSO-d $): \delta 10.15(1 \mathrm{H}, \mathrm{s})$,

$7.71(2 \mathrm{H}, \mathrm{d}, J=8.8 \mathrm{~Hz}), 7.50-7.31(5 \mathrm{H}, \mathrm{m}), 6.96(2 \mathrm{H}, \mathrm{d}, J=8.8 \mathrm{~Hz}), 5.21(2 \mathrm{H}, \mathrm{s}), 3.79(3 \mathrm{H}, \mathrm{s})$, $2.20(3 \mathrm{H}, \mathrm{s}) \cdot{ }^{13} \mathrm{C}$ NMR $(100 \mathrm{MHz}$, DMSO-d $)$ : (1 overlapping signal) $\delta 159.9,154.2,149.3,136.8$, 130.7, 128.4, 128.0, 127.5, 113.6, 65.9, 55.2, 13.8. HRMS (ESI) m/z: $[\mathrm{M}+\mathrm{H}]^{+}$Calculated for $\mathrm{C}_{17} \mathrm{H}_{19} \mathrm{~N}_{2} \mathrm{O}_{3} 299.1393$; found 299.1396.

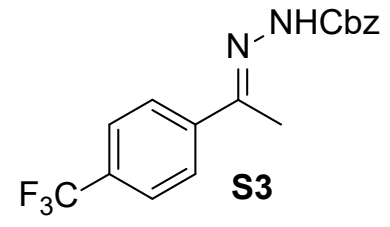

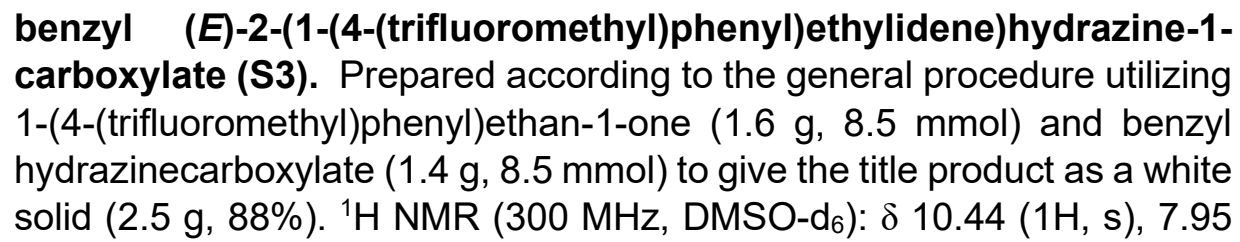
$(1 \mathrm{H}, \mathrm{d}, J=8.1 \mathrm{~Hz}), 7.76(1 \mathrm{H}, \mathrm{d}, J=8.3 \mathrm{~Hz}), 7.49-7.30(5 \mathrm{H}, \mathrm{m}), 5.22(2 \mathrm{H}, \mathrm{s}), 2.26(3 \mathrm{H}, \mathrm{s}) .{ }^{13} \mathrm{C}$ NMR (100 MHz, DMSO-d $)$ : (1 overlapping signal) $\delta$ 154.0, 147.6, 142.2, 136.6, $128.8\left(\mathrm{q}, J_{C-F}=\right.$ $31.8 \mathrm{~Hz}$ ), 128.4, 128.1, 126.7, $125.2\left(\mathrm{q}, J_{C-F}=3.8 \mathrm{~Hz}\right), 124.2\left(\mathrm{q}, J_{C-F}=272.0 \mathrm{~Hz}\right), 66.1,13.8$. HRMS (ESI) m/z: [M+H] $]^{+}$Calculated for $\mathrm{C}_{17} \mathrm{H}_{16} \mathrm{~N}_{2} \mathrm{O}_{2} \mathrm{~F}_{3}$ 337.1164; found 337.1160.

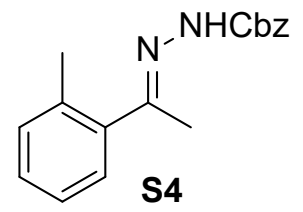

benzyl

(E)-2-(1-(o-tolyl)ethylidene)hydrazine-1-carboxylate

(S4). Prepared according to the general procedure utilizing 1-(o-tolyl)ethan-1-one $(0.948 \mathrm{~g}, 7.06 \mathrm{mmol})$ and benzyl hydrazinecarboxylate $(1.17 \mathrm{~g}, 7.06 \mathrm{mmol})$ to give the title product as a white solid $(0.977 \mathrm{~g}, 49 \%) .{ }^{1} \mathrm{H}$ NMR $(300 \mathrm{MHz}$, DMSO-d $\left._{6}\right): \delta 10.15(1 \mathrm{H}, \mathrm{s}), 7.48-7.13(9 \mathrm{H}, \mathrm{m}), 5.17(2 \mathrm{H}, \mathrm{s}), 2.29(3 \mathrm{H}, \mathrm{s}), 2.15$ $(3 \mathrm{H}, \mathrm{s}) \cdot{ }^{13} \mathrm{C}$ NMR $\left(100 \mathrm{MHz}, \mathrm{DMSO}-\mathrm{d}_{6}\right): \delta 154.2,152.1,139.9,136.7,135.1,130.5,128.4,128.4$, 128.1, 128.0, 127.9, 125.6, 65.9, 20.0, 18.0. HRMS (ESI) m/z: $[\mathrm{M}+\mathrm{H}]^{+}$Calculated for $\mathrm{C}_{17} \mathrm{H}_{19} \mathrm{~N}_{2} \mathrm{O}_{2}$ 283.1447; found 283.1442.<smiles>CC(=O)NN=C(C)c1cccc(Br)c1</smiles>

benzyl (E)-2-(1-(3-bromophenyl)ethylidene)hydrazine-1-carboxylate (S5). Prepared according to the general procedure utilizing 1-(3bromophenyl)ethan-1-one $(1.6 \mathrm{~g}, 8.1 \mathrm{mmol})$ and benzyl hydrazinecarboxylate $(1.35 \mathrm{~g}, 8.1 \mathrm{mmol})$ to give the title product as a white solid $(2.2 \mathrm{~g}, 78 \%) .{ }^{1} \mathrm{H}$ NMR (300 MHz, DMSO-d $): \delta 10.38(1 \mathrm{H}, \mathrm{s}), 7.91$ $(1 \mathrm{H}, \mathrm{s}), 7.72(1 \mathrm{H}, \mathrm{d}, J=7.8 \mathrm{~Hz}), 7.57(1 \mathrm{H}, \mathrm{d}, J=8.0 \mathrm{~Hz}), 7.48-7.29(6 \mathrm{H}, \mathrm{m}), 5.21(2 \mathrm{H}, \mathrm{s}), 2.21$ $(3 \mathrm{H}, \mathrm{s}) .{ }^{13} \mathrm{C}$ NMR $\left(100 \mathrm{MHz}, \mathrm{DMSO}-\mathrm{d}_{6}\right)$ : (2 overlapping signals) $\delta 154.1,147.6,140.6,136.6$, 
131.5, 130.5, 128.4, 128.0, 125.1, 121.8, 66.1, 13.8. HRMS (ESI) m/z: $[M+H]^{+}$Calculated for $\mathrm{C}_{16} \mathrm{H}_{16} \mathrm{~N}_{2} \mathrm{O}_{2} \mathrm{Br}$ 347.0395; found 347.0392.

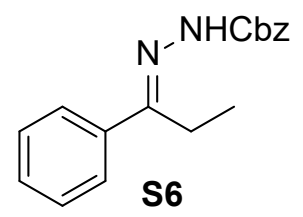

benzyl (E)-2-(1-phenylpropylidene)hydrazine-1-carboxylate

(S6).

Prepared according to the general procedure utilizing propiophenone $(1.13 \mathrm{~g}$, $8.4 \mathrm{mmol})$ and benzyl hydrazinecarboxylate $(1.4 \mathrm{~g}, 8.4 \mathrm{mmol})$ to give the title product as a white solid (2.3 g, 97\%). ${ }^{1} \mathrm{H}$ NMR (300 MHz, DMSO-d 6 ): $\delta 10.38$ $(1 \mathrm{H}, \mathrm{s}), 7.73(2 \mathrm{H}, \mathrm{dd}, J=7.9,2.0 \mathrm{~Hz}), 7.51-7.29(8 \mathrm{H}, \mathrm{m}), 5.20(2 \mathrm{H}, \mathrm{s}), 2.77$ $(2 \mathrm{H}, \mathrm{q}, J=7.4 \mathrm{~Hz}), 0.99(3 \mathrm{H}, \mathrm{t}, J=7.4 \mathrm{~Hz}) .{ }^{13} \mathrm{C}$ NMR $\left(100 \mathrm{MHz}, \mathrm{DMSO}-\mathrm{d}_{6}\right): \delta 154.2,153.2,137.1$, $136.7,128.8,128.4,128.4,128.0,128.0,126.1,66.0,19.3,10.7$. HRMS (ESI) m/z: $[M+H]^{+}$ Calculated for $\mathrm{C}_{17} \mathrm{H}_{19} \mathrm{~N}_{2} \mathrm{O}_{2} 283.1447$; found 283.1442 .

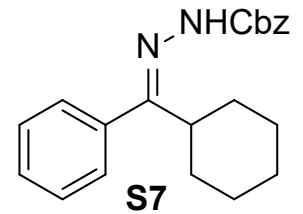

benzyl

(E)-2-(cyclohexyl(phenyl)methylene)hydrazine-1-carboxylate (S7). Prepared according to the general procedure utilizing cyclohexyl(phenyl)methanone (1.34 g, $7.13 \mathrm{mmol})$ and benzyl hydrazinecarboxylate $(1.19 \mathrm{~g}, 7.13 \mathrm{mmol})$ to give the title product as a 93:7, $(E):(Z)$ mixture $(1.8 \mathrm{~g}, 75 \%)$. A portion was purified by prep-HPLC to give pure $(E)$ and pure $(Z)$ isomers as white solids. ${ }^{1} \mathrm{H}$ NMR $\left(300 \mathrm{MHz}, \mathrm{DMSO}-\mathrm{d}_{6}\right)$ : (major, $\left.(E)\right):$ s), 7.52-7.39 (3H, m), 7.38-7.25 (5H, m), 7.24-7.12 (2H, m), $5.07(2 \mathrm{H}, \mathrm{s}), 2.48-2.36(1 \mathrm{H}, \mathrm{m}), 1.82-$ $1.52(5 \mathrm{H}, \mathrm{m}), 1.33-0.99(5 \mathrm{H}, \mathrm{m})$; (minor, $(\mathrm{Z}))$ : $\delta 10.44(1 \mathrm{H}, \mathrm{s}), 7.48-7.25(10 \mathrm{H}, \mathrm{m}), 5.16(2 \mathrm{H}, \mathrm{s})$, $3.11(1 \mathrm{H}, \mathrm{t}, J=11.4 \mathrm{~Hz}), 1.75-1.52(5 \mathrm{H}, \mathrm{m}), 1.49-0.98(5 \mathrm{H}, \mathrm{m}) .{ }^{13} \mathrm{C}$ NMR $\left(100 \mathrm{MHz}, \mathrm{DMSO}-\mathrm{d}_{6}\right)$ : (major, $(E) 1$ overlapping signal): $\delta 158.9,153.3,136.5,133.6,129.0,128.8,128.3,127.9,127.2$, 65.8, 45.3, 30.1, 25.6, 25.4; (minor, (Z) 1 overlapping signal): $\delta$ 158.3, 154.3, 138.2, 136.6, 128.4, 128.1, 128.0, 127.8, 127.7, 65.9, 37.2, 29.2, 25.4, 25.2. HRMS (ESI) m/z: [M+H] ${ }^{+}$Calculated for $\mathrm{C}_{21} \mathrm{H}_{25} \mathrm{~N}_{2} \mathrm{O}_{2}$ 337.1916; found 337.1917.

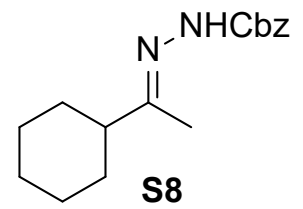

benzyl (E)-2-(1-cyclohexylethylidene)hydrazine-1-carboxylate (S8). Prepared according to the general procedure utilizing 1-cyclohexylethan-1-one $(0.931 \mathrm{~g}, 7.38 \mathrm{mmol})$ and benzyl hydrazinecarboxylate $(1.23 \mathrm{~g}, 7.38 \mathrm{mmol})$ to give the title product as a white solid $(1.7 \mathrm{~g}, 84 \%)$. ${ }^{1} \mathrm{H}$ NMR $(300 \mathrm{MHz}$, DMSO$\left.\mathrm{d}_{6}\right): \delta 9.69(1 \mathrm{H}, \mathrm{s}), 7.48-7.28(5 \mathrm{H}, \mathrm{m}), 5.12(2 \mathrm{H}, \mathrm{s}), 2.19-2.02(1 \mathrm{H}, \mathrm{m}), 1.75$ $(3 \mathrm{H}, \mathrm{s}), 1.74-1.55(5 \mathrm{H}, \mathrm{m}), 1.34-1.04(5 \mathrm{H}, \mathrm{m}) .{ }^{13} \mathrm{C}$ NMR $\left(100 \mathrm{MHz}, \mathrm{DMSO}-\mathrm{d}_{6}\right): \delta$ 158.0, 154.1, 136.9, 128.4, 128.0, 127.9, 65.6, 46.3, 29.7, 25.6, 25.6, 14.0. HRMS (ESI) m/z: [M+H] ${ }^{+}$Calculated for $\mathrm{C}_{16} \mathrm{H}_{23} \mathrm{~N}_{2} \mathrm{O}_{2} 275.1760$; found 275.1750 .

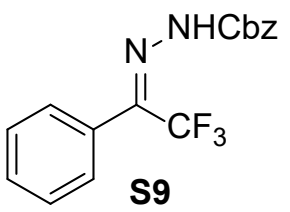

benzyl (Z)-2-(2,2,2-trifluoro-1-phenylethylidene)hydrazine-1-carboxylate (S9). Prepared according to the general procedure utilizing 2,2,2-trifluoro-1phenylethan-1-one $(2.79 \mathrm{~g}, 16 \mathrm{mmol})$ and benzyl hydrazinecarboxylate $(2.66$ $\mathrm{g}, 16 \mathrm{mmol})$ to give the title product as a white solid $(1.6 \mathrm{~g}, 31 \%) .{ }^{1} \mathrm{H}$ NMR $(300$ MHz, DMSO-d 6 ): $\delta 10.75(1 \mathrm{H}, \mathrm{s}), 7.59-7.47(3 \mathrm{H}, \mathrm{m}), 7.44-7.29(7 \mathrm{H}, \mathrm{m}), 5.17$ $(2 \mathrm{H}, \mathrm{s}) .{ }^{13} \mathrm{C}$ NMR (100 MHz, DMSO-d $): \delta 153.4,138.2\left(\mathrm{q}, J_{C-F}=33.6 \mathrm{~Hz}\right), 135.9,130.7,129.3$, 128.6, 128.4, 128.2, 128.1, 127.0, 120.8 (q, $\left.J_{C-F}=274.3 \mathrm{~Hz}\right), 66.7 . \quad \mathrm{HRMS}(\mathrm{ESI}) \mathrm{m} / \mathrm{z}:[\mathrm{M}+\mathrm{H}]^{+}$ Calculated for $\mathrm{C}_{16} \mathrm{H}_{14} \mathrm{~N}_{2} \mathrm{O}_{2} \mathrm{~F}_{3} 323.1007$; found 323.1002.

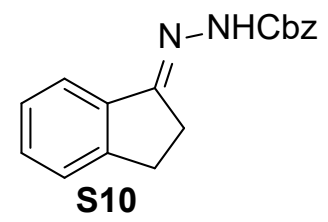

benzyl (E)-2-(2,3-dihydro-1H-inden-1-ylidene)hydrazine-1-carboxylate (S10). Prepared according to the general procedure utilizing 2,3-dihydro-1 $\mathrm{H}-$ inden-1-one $(1.10 \mathrm{~g}, 8.35 \mathrm{mmol})$ and benzyl hydrazinecarboxylate $(1.39 \mathrm{~g}$, $8.35 \mathrm{mmol})$ to give the title product as a white solid $(2.2 \mathrm{~g}, 94 \%) .{ }^{1} \mathrm{H}$ NMR (300 MHz, DMSO-d 6 ): $\delta 10.11(1 \mathrm{H}, \mathrm{s}), 7.60(1 \mathrm{H}, \mathrm{d}, J=7.4 \mathrm{~Hz}), 7.51-7.21$ 
$(8 \mathrm{H}, \mathrm{m}), 5.19(2 \mathrm{H}, \mathrm{s}), 3.12-2.95(2 \mathrm{H}, \mathrm{m}), 2.86-2.69(2 \mathrm{H}, \mathrm{m}) .{ }^{13} \mathrm{C}$ NMR $\left(100 \mathrm{MHz}, \mathrm{DMSO}-\mathrm{d}_{6}\right): \delta$ 158.7, 154.0, 148.1, 137.9, 136.7, 130.1, 128.4, 128.1, 128.0, 126.9, 125.6, 121.1, 65.9, 28.0, 27.1. HRMS (ESI) m/z: [M+H] ${ }^{+}$Calculated for $\mathrm{C}_{17} \mathrm{H}_{17} \mathrm{~N}_{2} \mathrm{O}_{2}$ 281.1290; found 281.1286.

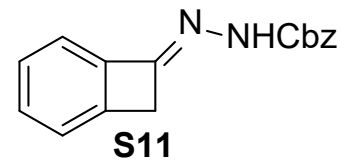

benzyl (E)-2-(bicyclo[4.2.0]octa-1(6),2,4-trien-7-ylidene)hydrazine-1carboxylate (S11). Prepared according to the general procedure utilizing bicyclo[4.2.0]octa-1(6),2,4-trien-7-one (1.03 $\mathrm{g}, 8.75 \mathrm{mmol})$ and benzyl hydrazinecarboxylate $(1.45 \mathrm{~g}, 8.75 \mathrm{mmol})$ to give the title product as a white solid (1.7 g, 73\%). ${ }^{1} \mathrm{H}$ NMR (300 MHz, DMSO-d $)$ : $\delta 10.72(1 \mathrm{H}, \mathrm{s}), 7.47-7.22(9 \mathrm{H}, \mathrm{m}), 5.18(2 \mathrm{H}$, s), $3.83(2 \mathrm{H}, \mathrm{s}) .{ }^{13} \mathrm{C}$ NMR $\left(75 \mathrm{MHz}, \mathrm{DMSO}-\mathrm{d}_{6}\right): \delta 153.9,148.6,144.0,142.8,136.6,131.0,128.4$, 128.3, 128.2, 128.1, 123.1, 118.8, 66.0, 40.9. HRMS (ESI) m/z: $[\mathrm{M}+\mathrm{H}]^{+}$Calculated for $\mathrm{C}_{16} \mathrm{H}_{15} \mathrm{~N}_{2} \mathrm{O}_{2}$ 267.1134; found 267.1142 .

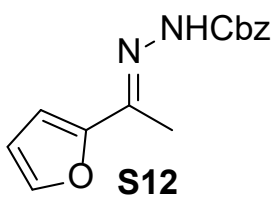

benzyl (E)-2-(1-(furan-2-yl)ethylidene)hydrazine-1-carboxylate (S12). Prepared according to the general procedure utilizing 1-(furan-2-yl)ethan-1one $(0.933 \mathrm{~g}, 8.47 \mathrm{mmol})$ and benzyl hydrazinecarboxylate $(1.41 \mathrm{~g}, 8.47 \mathrm{mmol})$ to give the title product as a white solid $(2.1 \mathrm{~g}, 96 \%) .{ }^{1} \mathrm{H}$ NMR $(300 \mathrm{MHz}$, DMSO- $\left.\mathrm{d}_{6}\right): \delta 10.19(1 \mathrm{H}, \mathrm{s}), 7.73(1 \mathrm{H}, \mathrm{s}), 7.51-7.28(5 \mathrm{H}, \mathrm{m}), 6.83(1 \mathrm{H}, \mathrm{d}, J=3.1$ $\mathrm{Hz}), 6.55(1 \mathrm{H}, \mathrm{dd}, J=3.1,1.7 \mathrm{~Hz}), 5.19(2 \mathrm{H}, \mathrm{s}), 2.13(3 \mathrm{H}, \mathrm{s}) .{ }^{13} \mathrm{C}$ NMR $\left(100 \mathrm{MHz}, \mathrm{DMSO}-\mathrm{d}_{6}\right): \delta$ $154.0,151.9,144.1,141.6,136.6,128.4,128.1,128.0,111.7,110.4,66.0,13.4$. HRMS (ESI) $\mathrm{m} / \mathrm{z}:[\mathrm{M}+\mathrm{H}]^{+}$Calculated for $\mathrm{C}_{14} \mathrm{H}_{15} \mathrm{~N}_{2} \mathrm{O}_{3}$ 259.1083; found 259.1086.

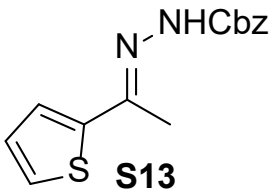

benzyl (E)-2-(1-(thiophen-2-yl)ethylidene)hydrazine-1-carboxylate (S13). Prepared according to the general procedure utilizing 1-(thiophen-2-yl)ethan1-one $(1.05 \mathrm{~g}, 8.35 \mathrm{mmol})$ and benzyl hydrazinecarboxylate $(1.39 \mathrm{~g}, 8.35$ $\mathrm{mmol})$ to give the title product as a white solid $(2.2 \mathrm{~g}, 96 \%) .{ }^{1} \mathrm{H}$ NMR $(300 \mathrm{MHz}$, DMSO- $\left._{6}\right): \delta 10.23(1 \mathrm{H}, \mathrm{s}), 7.53(1 \mathrm{H}, \mathrm{d}, \mathrm{J}=4.7 \mathrm{~Hz}), 7.47-7.29(6 \mathrm{H}, \mathrm{m}), 7.05$ $(1 \mathrm{H}, \mathrm{dd}, \mathrm{J}=4.9,3.8 \mathrm{~Hz}), 5.18(2 \mathrm{H}, \mathrm{s}), 2.24(3 \mathrm{H}, \mathrm{s}) .{ }^{13} \mathrm{C}$ NMR $\left(100 \mathrm{MHz}, \mathrm{DMSO}-\mathrm{d}_{6}\right)$ : (1 overlapping signal) $\delta 153.8,146.3,143.5,136.6,128.4,128.1,128.0,127.4,127.1,65.9,14.3$. HRMS (ESI) $\mathrm{m} / \mathrm{z}:[\mathrm{M}+\mathrm{H}]^{+}$Calculated for $\mathrm{C}_{14} \mathrm{H}_{15} \mathrm{~N}_{2} \mathrm{O}_{2} \mathrm{~S} 275.0854$; found 275.0853 .

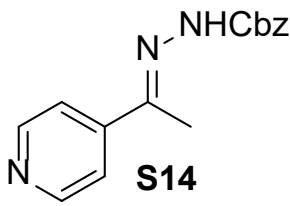

benzyl (E)-2-(1-(pyridin-4-yl)ethylidene)hydrazine-1-carboxylate (S14). Prepared according to the general procedure utilizing 1-(pyridin-4-yl)ethan-1one $(1.00 \mathrm{~g}, 8.30 \mathrm{mmol})$ and benzyl hydrazinecarboxylate $(1.38 \mathrm{~g}, 8.30 \mathrm{mmol})$ to give the title product as a white solid $(2.1 \mathrm{~g}, 94 \%) .{ }^{1} \mathrm{H}$ NMR $(300 \mathrm{MHz}$, DMSO-d 6$): \delta 10.52(1 \mathrm{H}, \mathrm{s}), 8.59(2 \mathrm{H}, \mathrm{d}, \mathrm{J}=6.0 \mathrm{~Hz}), 7.67(2 \mathrm{H}, \mathrm{d}, \mathrm{J}=6.1 \mathrm{~Hz})$, 7.50-7.29 (5H, m), $5.22(2 \mathrm{H}, \mathrm{s}), 2.22(3 \mathrm{H}, \mathrm{s}) .{ }^{13} \mathrm{C}$ NMR $\left(100 \mathrm{MHz}, \mathrm{DMSO}-\mathrm{d}_{6}\right): \delta$ 172.0, 154.0, 149.9, 146.8, 145.2, 136.5, 128.5, 128.1, 120.2, 66.2, 13.3. HRMS (ESI) m/z: $[\mathrm{M}+\mathrm{H}]^{+}$Calculated for $\mathrm{C}_{15} \mathrm{H}_{16} \mathrm{~N}_{3} \mathrm{O}_{2} 270.1243$; found 270.1238 .

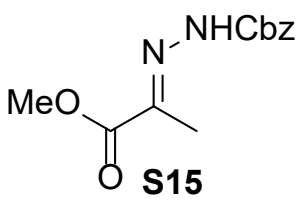

benzyl (E)-2-(1-methoxy-1-oxopropan-2-ylidene)hydrazine-1-carboxylate (S15). Prepared according to the general procedure utilizing methyl 2oxopropanoate $(0.765 \mathrm{~g}, 7.49 \mathrm{mmol})$ and benzyl hydrazinecarboxylate (1.25 $\mathrm{g}, 7.49 \mathrm{mmol})$ to give the title product as a white solid $(1.5 \mathrm{~g}, 80 \%) .{ }^{1} \mathrm{H}$ NMR (300 MHz, DMSO-d $): \delta 10.64(1 \mathrm{H}, \mathrm{s}), 7.48-7.28(5 \mathrm{H}, \mathrm{m}), 5.22(2 \mathrm{H}, \mathrm{s}), 3.72$ $(3 \mathrm{H}, \mathrm{s}), 2.02(3 \mathrm{H}, \mathrm{s}) .{ }^{13} \mathrm{C}$ NMR $\left(100 \mathrm{MHz}, \mathrm{DMSO}-\mathrm{d}_{6}\right): \delta 164.9,153.5,141.0,136.1,128.5,128.2$, 128.2, 66.6, 52.2, 12.9. HRMS (ESI) m/z: $[\mathrm{M}+\mathrm{H}]^{+}$Calculated for $\mathrm{C}_{12} \mathrm{H}_{15} \mathrm{~N}_{2} \mathrm{O}_{4}$ 251.1032; found 251.1022 . 


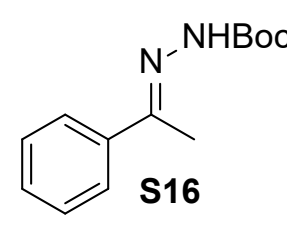

tert-butyl (E)-2-(1-phenylethylidene)hydrazine-1-carboxylate

(S16). Prepared according to the general procedure utilizing acetophenone $(0.697 \mathrm{~g}$, $5.80 \mathrm{mmol}$ ) and tert-butyl hydrazinecarboxylate $(0.766 \mathrm{~g}, 5.80 \mathrm{mmol})$ to give the title product as a white solid $(1.25 \mathrm{~g}, 92 \%)$. All spectral data are in accordance with the literature. ${ }^{3}$

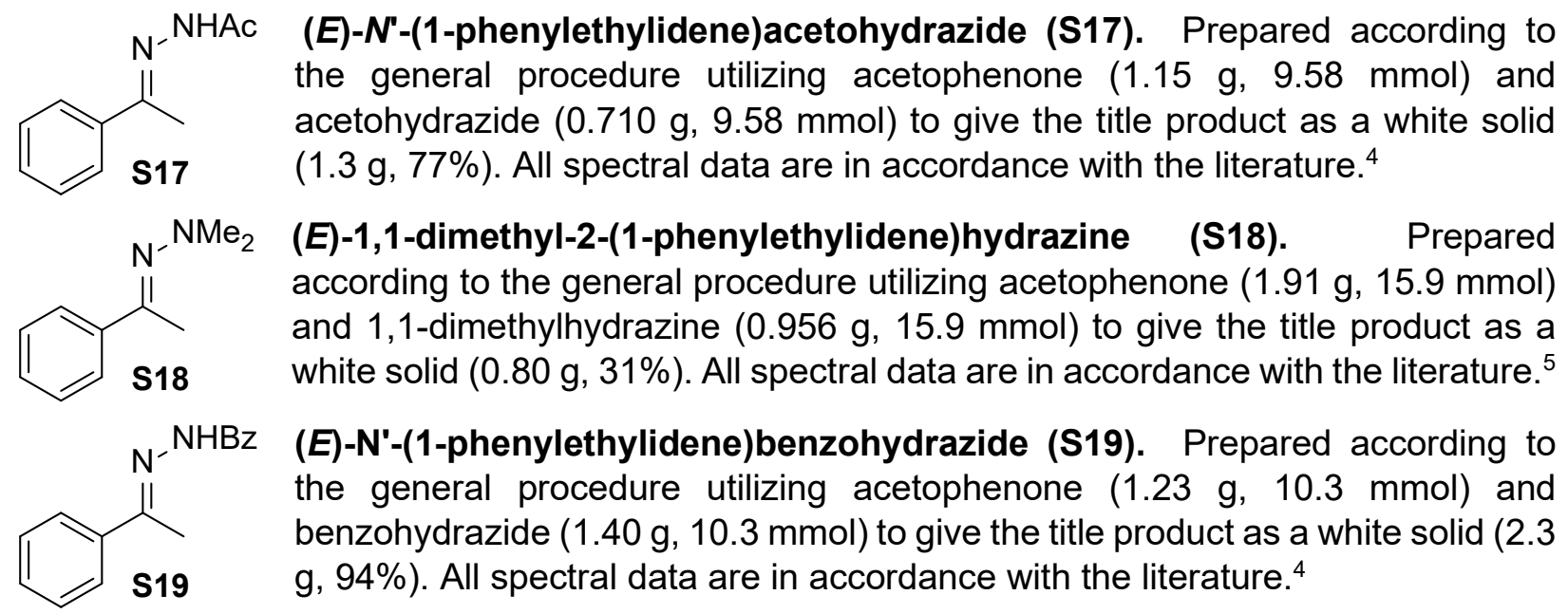

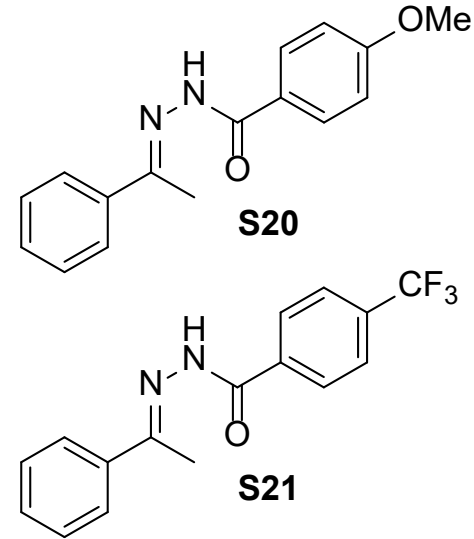

(E)-4-methoxy- $\mathrm{N}^{\prime}$-(1-phenylethylidene)benzohydrazide (S20). Prepared according to the general procedure utilizing acetophenone $(1.20 \mathrm{~g}, 10.0 \mathrm{mmol})$ and 4-methoxybenzohydrazide $(1.66 \mathrm{~g}, 10.0 \mathrm{mmol})$ to give the title product as a white solid $(1.8 \mathrm{~g}$, $67 \%$ ). All spectral data are in accordance with the literature. ${ }^{6}$

(E)-N'-(1-phenylethylidene)-4-(trifluoromethyl)benzohydrazide (S21). Prepared according to the general procedure utilizing acetophenone $(1.20 \mathrm{~g}, 10.0 \mathrm{mmol})$ and 4-(trifluoromethyl) benzohydrazide $(2.04 \mathrm{~g}, 10.0 \mathrm{mmol})$ to give the title product as a white solid $(2.2 \mathrm{~g}, 72 \%)$. All spectral data are in accordance with the literature. ${ }^{7}$ 


\subsection{Representative Procedure for Hydrazone Hydrogenation}

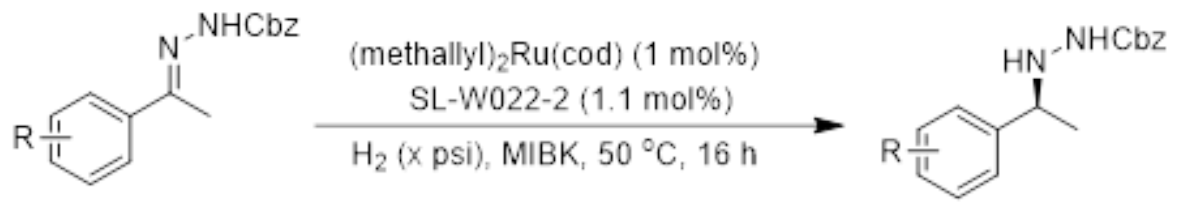

To a vial equipped with stir bar in a nitrogen filled glovebox was added bis(2-methallyl)(1,5cyclooctadiene)ruthenium(II) (1 mol\%) followed by Walphos ligand SL-W022-1 (1.1 mol\%) and dry DCE $(0.05 \mathrm{M})$. The resulting solution was stirred for 20 minutes at room temperature resulting in a golden solution. $\mathrm{HBF}_{4}-\mathrm{OEt}_{2}(2 \mathrm{~mol} \%)$ was added and the resulting dark maroon solution was stirred at room temperature for an additional 20 minutes. The solvent was then removed under vacuum to give a dark solid. The hydrazone substrate (1 equivalent) was then added to the catalyst containing vial as a slurry in dry $\operatorname{MIBK}(0.2 \mathrm{M})$. The resulting mixture was heated to 50 ${ }^{\circ} \mathrm{C}$ in a metal block under the indicated hydrogen pressure for 14 hours. The reaction yield was determined by quantitative HPLC analysis of the reaction mixture. The enantioselectivity of the reaction was determined by chiral SFC analysis. 


\subsection{Synthesis and Characterization of Hydrazine Products}<smiles>CC(=O)NNC(C)c1ccc(C(F)(F)F)nc1</smiles>

benzyl (S)-2-(1-(6-(trifluoromethyl)pyridin-3-yl)ethyl)hydrazine-1carboxylate (compound 2, Table 1). Prepared according to the general procedure utilizing benzyl (E)-2-(1-(6-(trifluoromethyl)pyridin-3yl)ethylidene)hydrazine-1-carboxylate (337 mg, $1.00 \mathrm{mmol})$, bis(2methallyl)(1,5-cyclooctadiene)ruthenium(II) (3.2 mg, $1 \mathrm{~mol} \%)$, Walphos ligand SL-W022-1 (7.6 mg, 1.1 mol\%), $\mathrm{HBF}_{4}-\mathrm{OEt}_{2}(2.7 \mu \mathrm{L}, 2 \mathrm{~mol} \%)$ and hydrogen (100 psi) to give the title product $(92 \%, 98 \%$ ee $) .{ }^{1} \mathrm{H}$ NMR $\left(400 \mathrm{MHz}, \mathrm{DMSO}-\mathrm{d}_{6}\right): \delta 8.71(1 \mathrm{H}, \mathrm{s}), 8.64(1 \mathrm{H}, \mathrm{s})$, $8.02(1 \mathrm{H}, \mathrm{d}, J=7.7 \mathrm{~Hz}), 7.83(1 \mathrm{H}, \mathrm{d}, J=7.8 \mathrm{~Hz}), 7.40-7.17(5 \mathrm{H}, \mathrm{m}), 5.18(1 \mathrm{H}, \mathrm{s}), 5.07-4.90(2 \mathrm{H}$, $\mathrm{m}), 4.31(1 \mathrm{H}, \mathrm{s}), 1.24(3 \mathrm{H}, \mathrm{d}, J=6.6 \mathrm{~Hz}) .{ }^{13} \mathrm{C}$ NMR $\left(100 \mathrm{MHz}, \mathrm{DMSO}-\mathrm{d}_{6}\right): \delta 156.8,149.4,145.1$ $\left(\mathrm{q}, J_{C-F}=33.5 \mathrm{~Hz}\right), 143.7,137.0,136.8,128.3,127.8,127.6,121.8\left(\mathrm{q}, J_{C-F}=274.0 \mathrm{~Hz}\right), 120.3(\mathrm{q}$, $\left.J_{C-F}=2.4 \mathrm{~Hz}\right), 65.3,56.0,21.2$. HRMS $(E S I) \mathrm{m} / \mathrm{z}:[\mathrm{M}+\mathrm{H}]^{+}$Calculated for $\mathrm{C}_{16} \mathrm{H}_{17} \mathrm{~N}_{3} \mathrm{O}_{2} \mathrm{~F}_{3}[\mathrm{M}+\mathrm{H}]^{+}$ 340.1273 ; found 340.1275 .

Chiral SFC analysis of 2 (Chiralpak IA, $35^{\circ} \mathrm{C}, 100$ bar, cosolvent: $0.1 \%$ DEA in $\mathrm{MeOH}, 4 \mathrm{~mL} / \mathrm{min}$, 0 min to $2.5 \mathrm{~min}$ ramp $10-40 \%$ cosolvent then hold $40 \%$ cosolvent for $2 \mathrm{~min}$ )

Racemate:

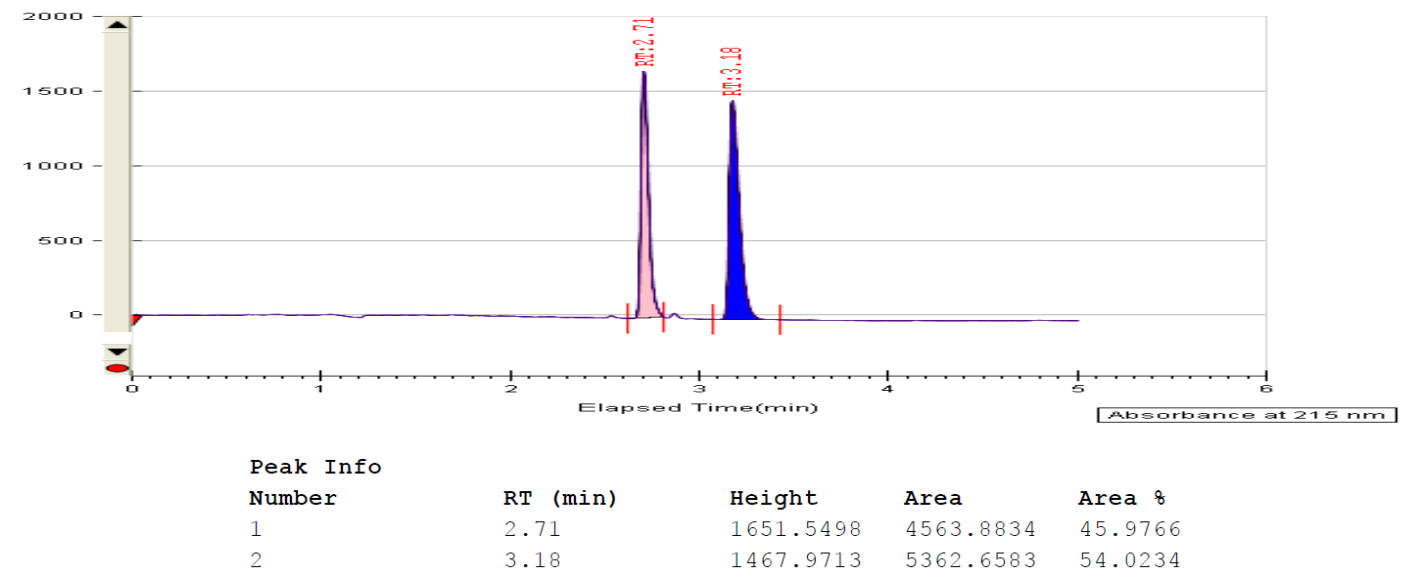

Reaction Product:

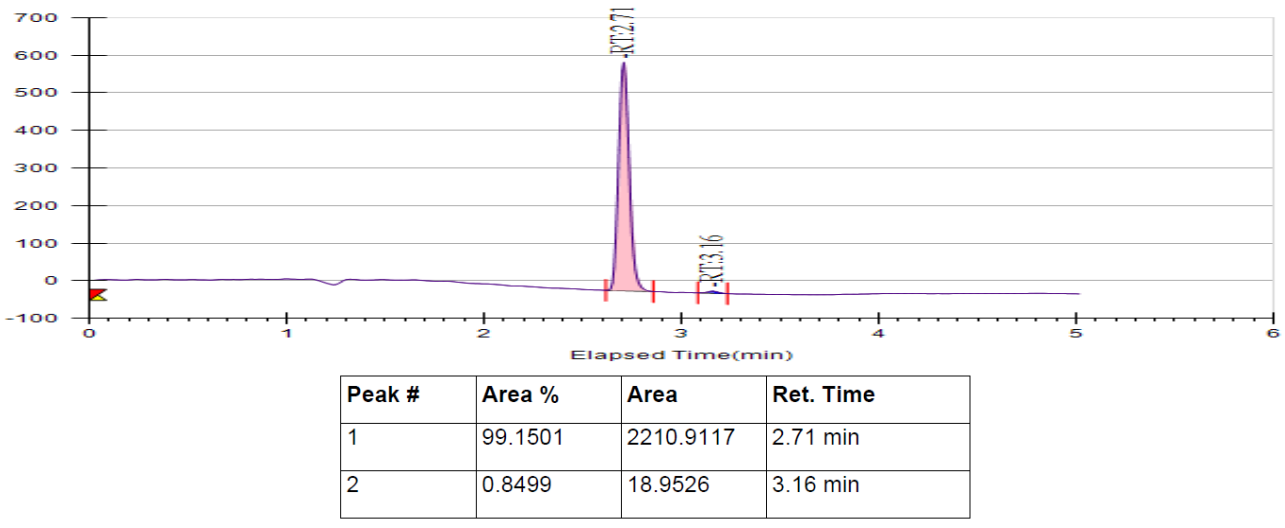



2). Prepared according to the general procedure utilizing benzyl (E)-2-(1phenylethylidene)hydrazine-1-carboxylate (268 mg, $1.00 \mathrm{mmol})$, bis(2methallyl)(1,5-cyclooctadiene)ruthenium(II) (3.2 mg, $1 \mathrm{~mol} \%)$, Walphos ligand SL-W022-1 (7.6 mg, 1.1 mol\%), $\mathrm{HBF}_{4}-\mathrm{OEt}_{2}(2.7 \mu \mathrm{L}, 2 \mathrm{~mol} \%)$ and hydrogen (100 psi) to give the title product $\left(95 \%, 97 \%\right.$ ee). ${ }^{1} \mathrm{H}$ NMR $\left(400 \mathrm{MHz}, \mathrm{DMSO}-\mathrm{d}_{6}\right): \delta 8.56(1 \mathrm{H}, \mathrm{s})$, 7.43-7.16 $(10 \mathrm{H}, \mathrm{m}), 5.01(2 \mathrm{H}, \mathrm{s}), 4.79(1 \mathrm{H}, \mathrm{s}), 4.12(1 \mathrm{H}, \mathrm{s}), 1.18(3 \mathrm{H}, \mathrm{d}, J=6.5 \mathrm{~Hz}) .{ }^{13} \mathrm{C} \mathrm{NMR}$ (100 MHz, DMSO-d $\left.\mathrm{d}_{6}\right): \delta 156.9,144.2,137.1,128.3,128.1,127.8,127.6,127.0,126.8,65.2,58.2$, 21.7. HRMS (ESI) m/z: [M+H] ${ }^{+}$Calculated for $\mathrm{C}_{16} \mathrm{H}_{19} \mathrm{~N}_{2} \mathrm{O}_{2}$ 271.1447; found 271.1446.

Chiral SFC analysis of 3 (Chiralpak IA, $35{ }^{\circ} \mathrm{C}, 100$ bar, cosolvent: $0.1 \%$ DEA in $\mathrm{MeOH}, 4 \mathrm{~mL} / \mathrm{min}$, 0 min to $2.5 \mathrm{~min}$ ramp $10-40 \%$ cosolvent then hold $40 \%$ cosolvent for $2 \mathrm{~min}$ )

Racemate:

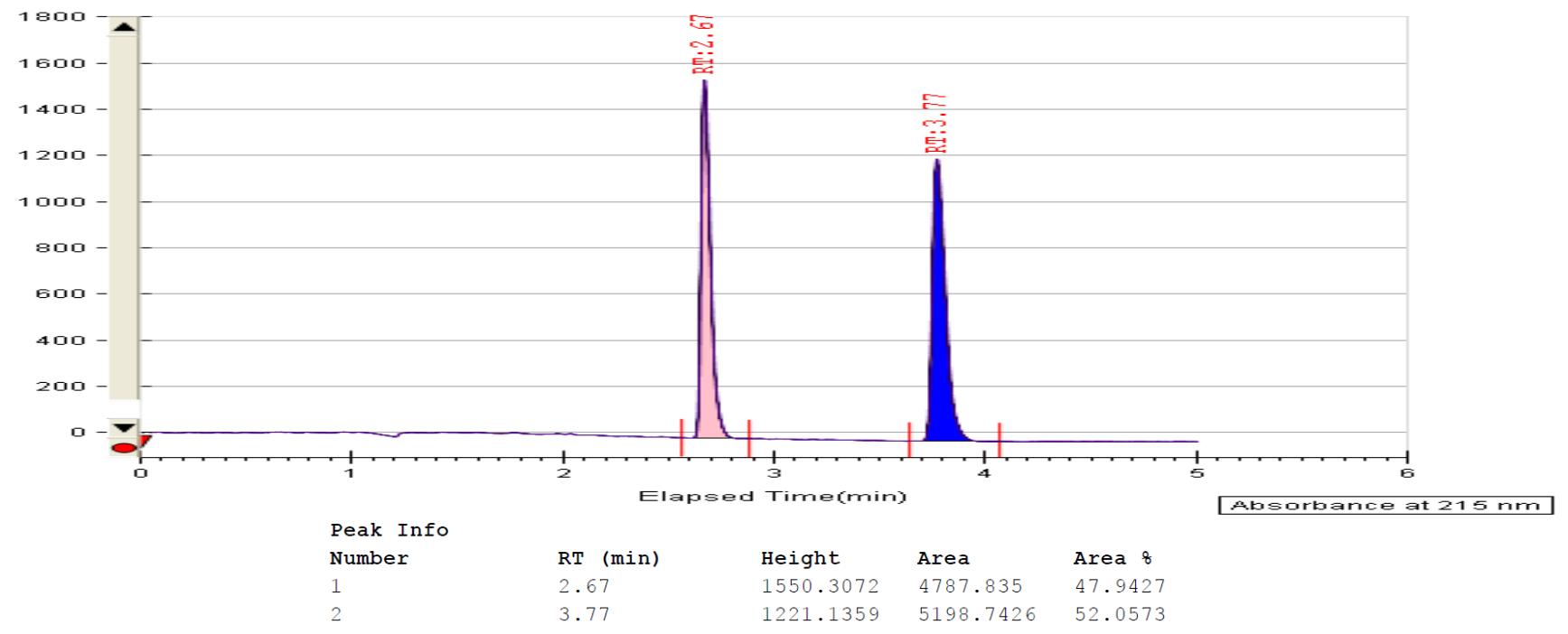

Reaction Product:

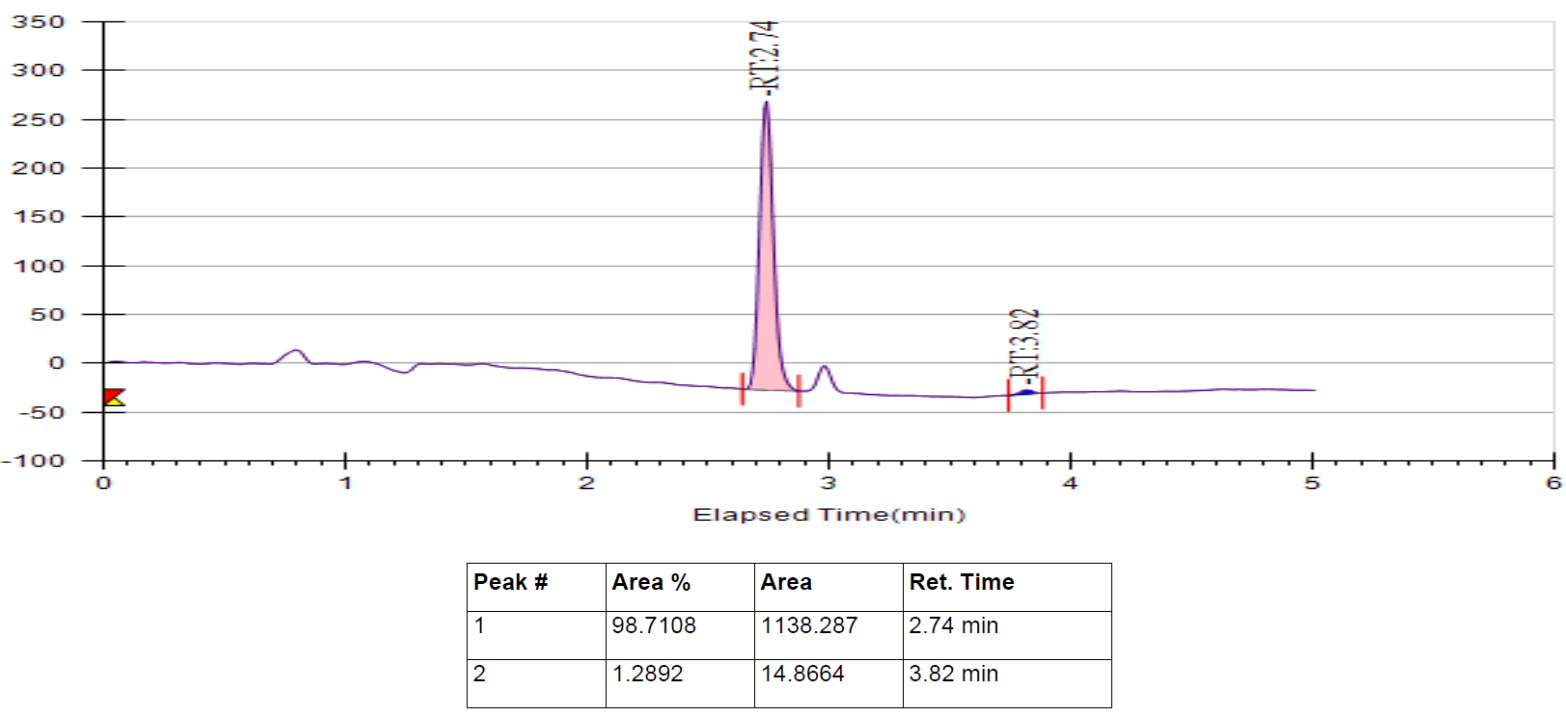


<smiles>COc1ccc([C@H](C)NC(=O)OCc2ccccc2)cc1</smiles>

benzyl (S)-2-(1-(4-methoxyphenyl)ethyl)hydrazine-1-carboxylate (compound 4, Table 2). Prepared according to the general procedure utilizing benzyl benzyl (E)-2-(1-(4-methoxyphenyl)ethylidene)hydrazine1-carboxylate (298 mg, $1.00 \mathrm{mmol})$, bis(2-methallyl)(1,5cyclooctadiene)ruthenium(II) $(3.2 \mathrm{mg}, 1 \mathrm{~mol} \%)$, Walphos ligand SLW022-1 (7.6 mg, 1.1 mol\%), $\mathrm{HBF}_{4}-\mathrm{OEt}_{2}(2.7 \mu \mathrm{L}, 2 \mathrm{~mol} \%)$ and hydrogen (100 psi) to give the title product $\left(89 \%, 96 \%\right.$ ee). ${ }^{1} \mathrm{H}$ NMR $\left(400 \mathrm{MHz}, \mathrm{DMSO}_{\mathrm{d}}\right): \delta 8.53(1 \mathrm{H}, \mathrm{s}), 7.41-7.26(5 \mathrm{H}, \mathrm{m}), 7.23$ $(2 \mathrm{H}, \mathrm{d}, J=8.2 \mathrm{~Hz}), 6.85(2 \mathrm{H}, \mathrm{d}, J=8.2 \mathrm{~Hz}), 5.01(2 \mathrm{H}, \mathrm{s}), 4.69(1 \mathrm{H}, \mathrm{s}), 4.06(1 \mathrm{H}, \mathrm{s}), 3.72(3 \mathrm{H}, \mathrm{s})$, $1.16(3 \mathrm{H}, \mathrm{d}, J=6.5 \mathrm{~Hz}) .{ }^{13} \mathrm{C}$ NMR $\left(100 \mathrm{MHz}, \mathrm{DMSO}-\mathrm{d}_{6}\right): \delta 158.3,156.9,137.1,136.0,128.3$, 128.1, 127.8, 127.6, 113.5, 65.2, 57.6, 55.0, 21.6. HRMS (ESI) m/z: $[\mathrm{M}+\mathrm{H}]^{+}$Calculated for $\mathrm{C}_{17} \mathrm{H}_{21} \mathrm{~N}_{2} \mathrm{O}_{3} 301.1552$; found 301.1556 .

Chiral SFC analysis of 4 (Chiralpak IA, $35^{\circ} \mathrm{C}, 100$ bar, cosolvent: $20 \mathrm{mM} \mathrm{NH}_{3}$ in $\mathrm{MeOH}, 2 \mathrm{~mL} / \mathrm{min}$, $0 \mathrm{~min}$ to $4 \mathrm{~min}$ ramp $10-50 \%$ cosolvent then hold $50 \%$ cosolvent for $2 \mathrm{~min}$ )

Racemate:

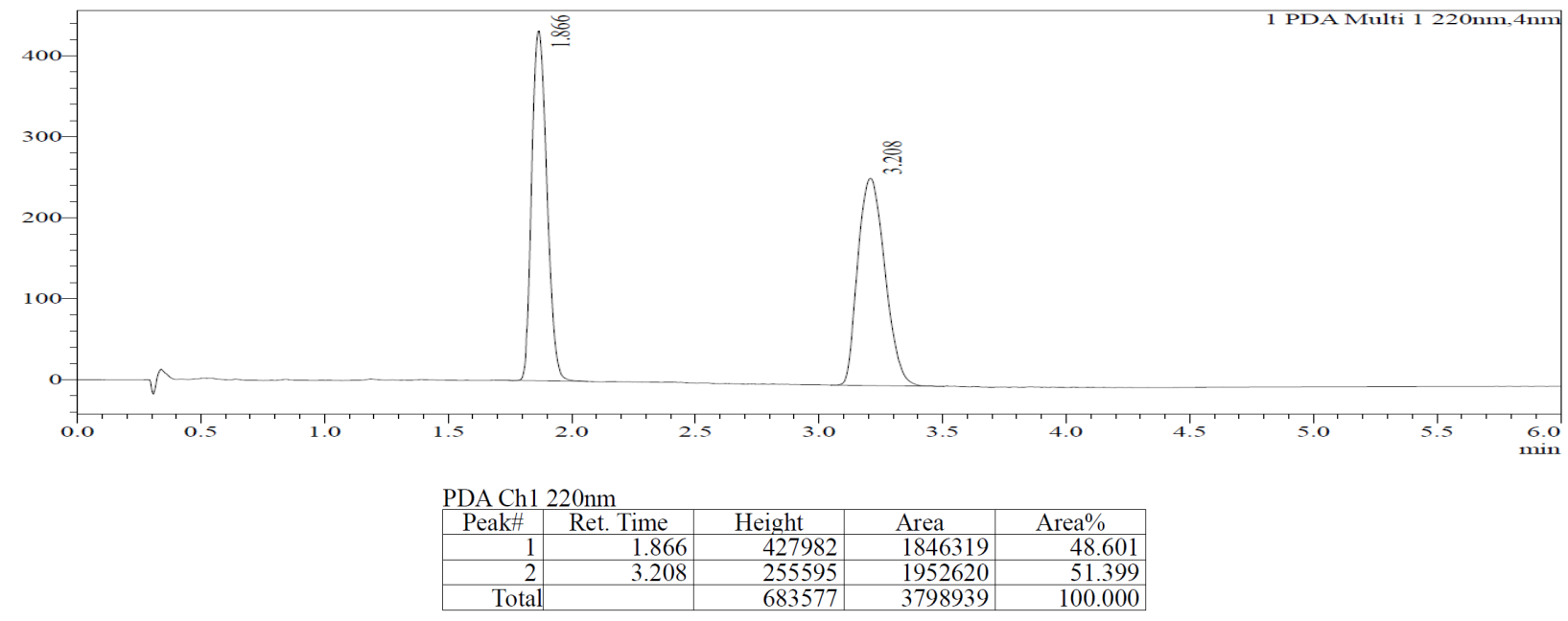

Reaction Product:

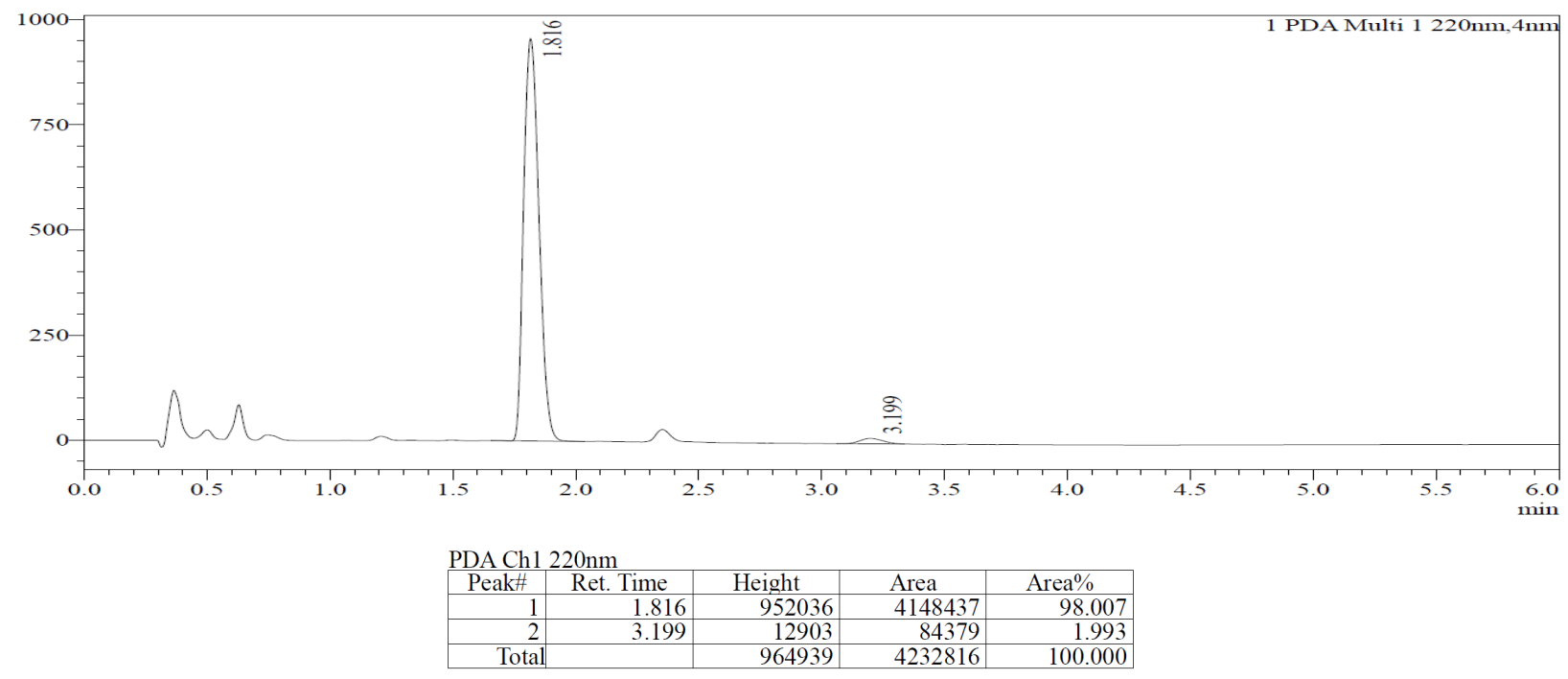


<smiles>CC(=O)NC(C)c1ccc(C(F)(F)F)cc1</smiles>

(S)-2-(1-(4-(trifluoromethyl)phenyl)ethyl)hydrazine-1carboxylate (compound 5, Table 2). Prepared according to the general procedure utilizing benzyl (E)-2-(1-(4-(trifluoromethyl) phenyl)ethylidene)hydrazine-1-carboxylate (336 mg, $1.00 \mathrm{mmol})$, bis(2methallyl)(1,5-cyclooctadiene)ruthenium(II) $(3.2 \mathrm{mg}, 1 \mathrm{~mol} \%)$, Walphos ligand SL-W022-1 (7.6 mg, 1.1 mol\%), $\mathrm{HBF}_{4}-\mathrm{OEt}_{2}(2.7 \mu \mathrm{L}, 2 \mathrm{~mol} \%)$ and hydrogen (100 psi) to give the title product $\left(98 \%, 95 \%\right.$ ee). ${ }^{1} \mathrm{H}$ NMR $\left(400 \mathrm{MHz}, \mathrm{DMSO}-\mathrm{d}_{6}\right): \delta 8.59(1 \mathrm{H}, \mathrm{s}), 7.65(2 \mathrm{H}, \mathrm{d}$, $J=7.7 \mathrm{~Hz}), 7.56(2 \mathrm{H}, \mathrm{d}, J=7.7 \mathrm{~Hz}), 7.42-7.20(5 \mathrm{H}, \mathrm{m}), 5.14-4.93(3 \mathrm{H}, \mathrm{m}), 4.23(1 \mathrm{H}, \mathrm{s}), 1.20(3 \mathrm{H}$, $\mathrm{d}, J=6.5 \mathrm{~Hz}$ ). ${ }^{13} \mathrm{C}$ NMR $\left(100 \mathrm{MHz}\right.$, DMSO-d $\left.\mathrm{d}_{6}\right)$ : (1 overlapping signal) $\delta$ 156.8, 149.2, 137.0, 128.3, 127.8, 127.8, 127.6, 124.9 (q, $J_{C-F}=4.0 \mathrm{~Hz}$ ), 124.4 (q, $\left.J_{C-F}=272.0 \mathrm{~Hz}\right), 65.2,57.9$, 21.6. HRMS (ESI) m/z: $[\mathrm{M}+\mathrm{H}]^{+}$Calculated for $\mathrm{C}_{17} \mathrm{H}_{18} \mathrm{~N}_{2} \mathrm{O}_{2} \mathrm{~F}_{3}$ 339.1320; found 339.1320.

Chiral SFC analysis of 5 (Chiralpak IA, $35^{\circ} \mathrm{C}, 100$ bar, cosolvent: $0.1 \%$ DEA in $\mathrm{MeOH}, 4 \mathrm{~mL} / \mathrm{min}$, 0 min to 2.5 min ramp $10-40 \%$ cosolvent then hold $40 \%$ cosolvent for 2 min)

Racemate:

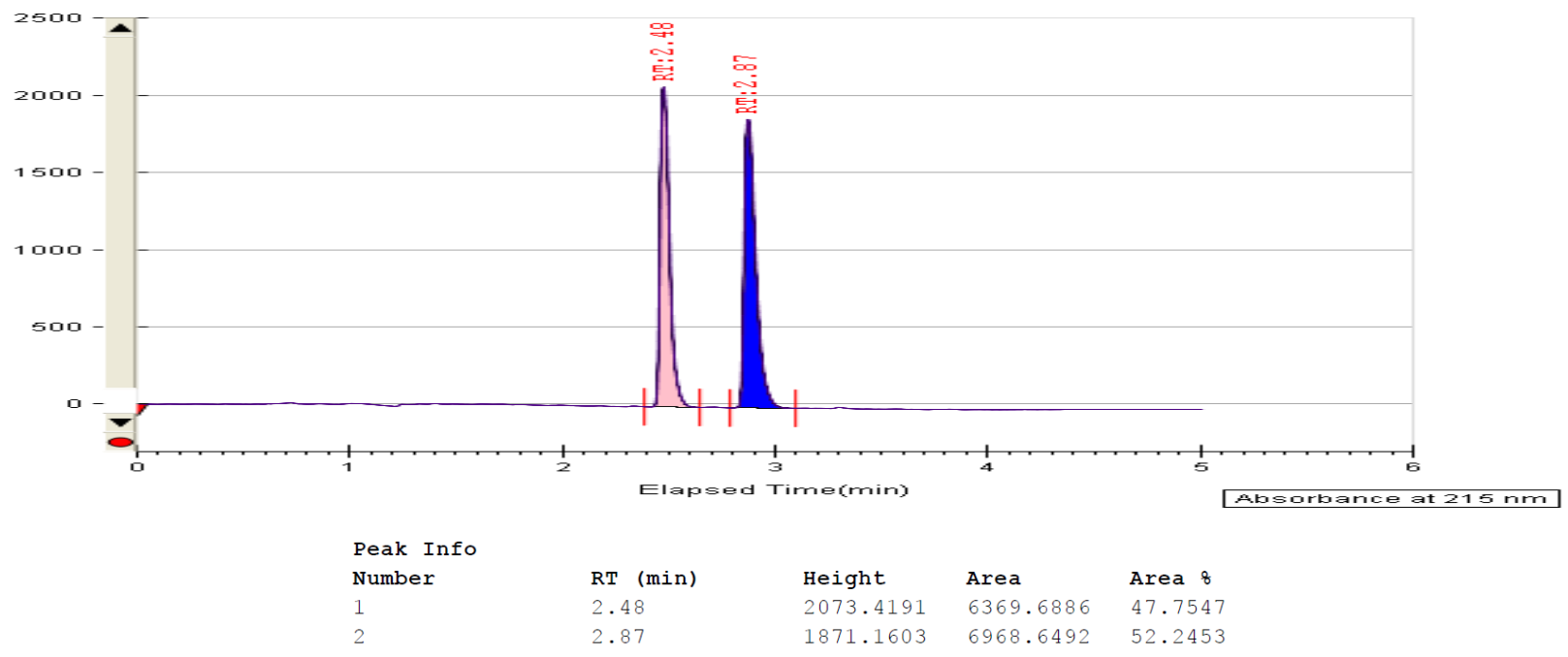

Reaction Product:

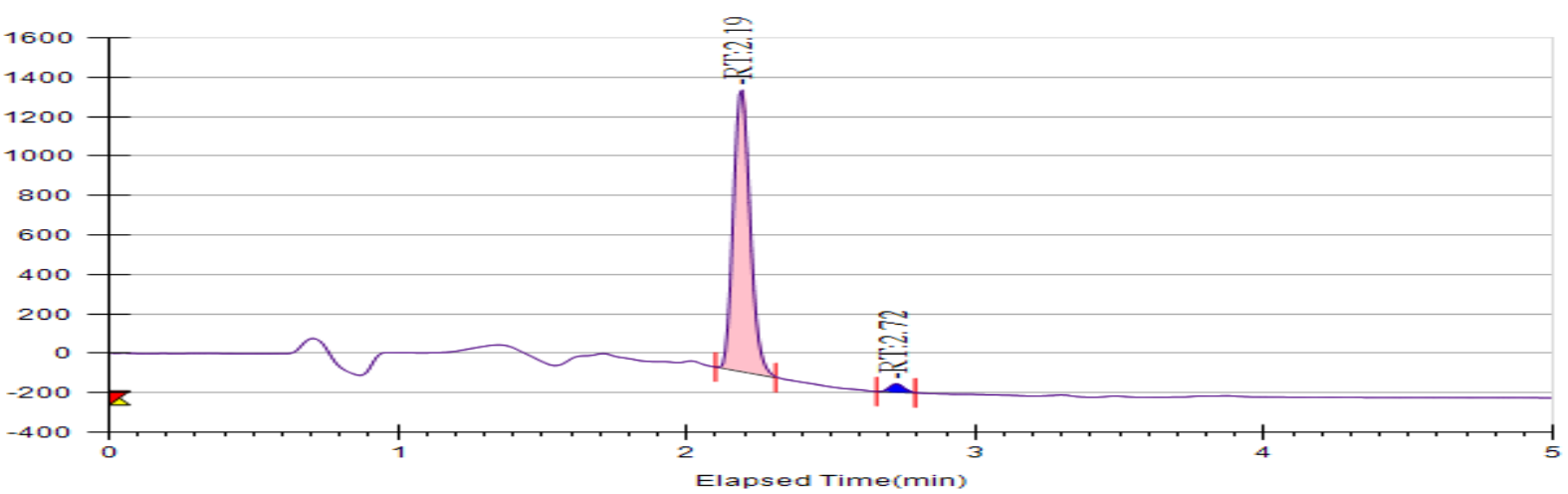

\begin{tabular}{|l|l|l|l|}
\hline Peak \# & Area \% & Area & Ret. Time \\
\hline 1 & 97.5819 & 5555.5199 & $2.19 \min$ \\
\hline 2 & 2.4181 & 137.6684 & $2.72 \mathrm{~min}$ \\
\hline
\end{tabular}




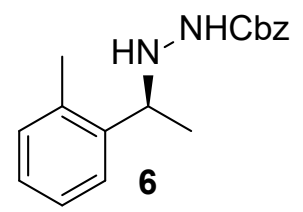

benzyl (S)-2-(1-(o-tolyl)ethyl)hydrazine-1-carboxylate (compound 6, Table 2). Prepared according to the general procedure utilizing benzyl $(E)-2-$ (1-(o-tolyl)ethylidene)hydrazine-1-carboxylate $(282 \mathrm{mg}, 1.00 \mathrm{mmol})$, bis(2methallyl)(1,5-cyclooctadiene)ruthenium(II) $(3.2 \mathrm{mg}, 1 \mathrm{~mol} \%)$, Walphos ligand SL-W022-1 (7.6 mg, $\left.1.1 \mathrm{~mol}^{\circ}\right), \mathrm{HBF}_{4}-\mathrm{OEt}_{2}(2.7 \mu \mathrm{L}, 2 \mathrm{~mol} \%)$ and hydrogen (200 psi) to give the title product $\left(95 \%, 55 \%\right.$ ee). ${ }^{1} \mathrm{H}$ NMR $\left(400 \mathrm{MHz}, \mathrm{DMSO}-\mathrm{d}_{6}\right): \delta 8.57(1 \mathrm{H}, \mathrm{s})$, $7.48(1 \mathrm{H}, \mathrm{d}, J=7.6 \mathrm{~Hz}), 7.43-7.23(5 \mathrm{H}, \mathrm{m}), 7.20-7.04(3 \mathrm{H}, \mathrm{m}), 5.11-4.95(2 \mathrm{H}, \mathrm{m}), 4.73(1 \mathrm{H}, \mathrm{s})$, $4.40(1 \mathrm{H}, \mathrm{s}), 2.26(3 \mathrm{H}, \mathrm{s}), 1.15(3 \mathrm{H}, \mathrm{d}, J=6.4 \mathrm{~Hz}) .{ }^{13} \mathrm{C} \mathrm{NMR}\left(100 \mathrm{MHz}, \mathrm{DMSO}-\mathrm{d}_{6}\right): \delta 156.9$, 142.0, 137.1, 135.2, 130.0, 128.3, 127.8, 127.6, 126.4, 126.1, 126.0, 65.2, 53.8, 20.4, 18.7. HRMS (ESI) m/z: [M+H] $]^{+}$Calculated for $\mathrm{C}_{17} \mathrm{H}_{21} \mathrm{~N}_{2} \mathrm{O}_{2}$ 285.1603; found 285.1603.

Chiral SFC analysis of 6 (Chiralpak IA, $35^{\circ} \mathrm{C}, 100$ bar, cosolvent: $20 \mathrm{mM} \mathrm{NH}_{3}$ in $\mathrm{MeOH}, 4 \mathrm{~mL} / \mathrm{min}$, 0 min to 4 min ramp $10-50 \%$ cosolvent then hold $50 \%$ cosolvent for $2 \mathrm{~min}$ )

Racemate:
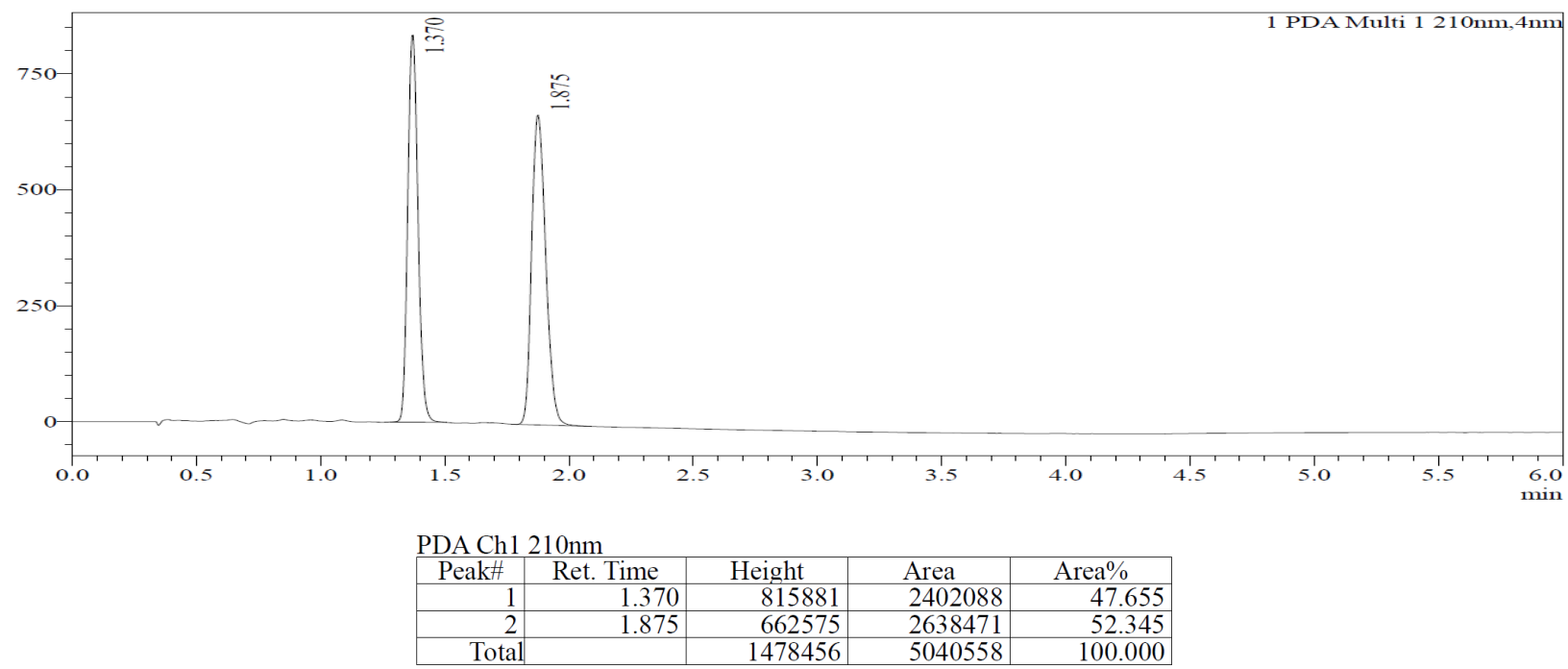

Reaction Product:

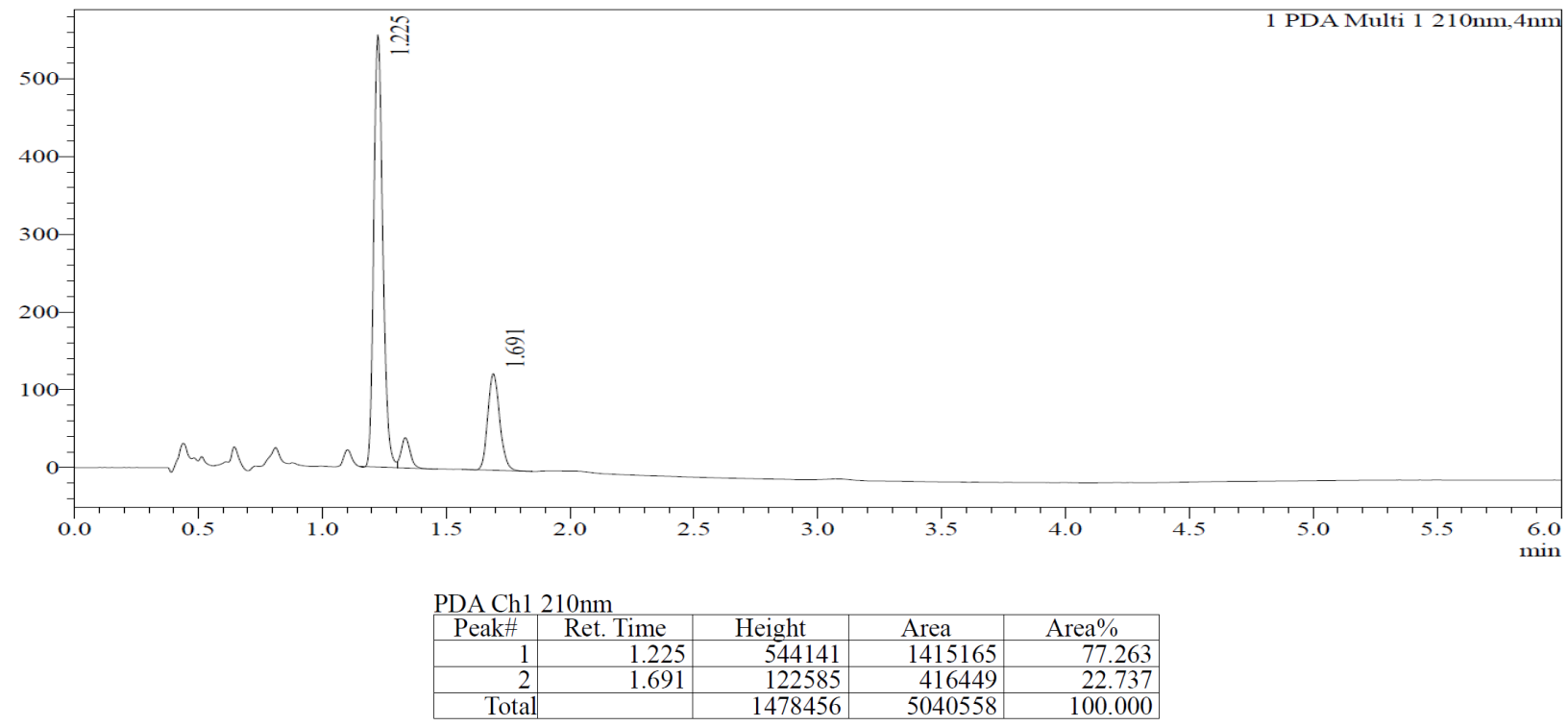


<smiles>CC(=O)NNC(C)c1cccc(Br)c1</smiles>

(S)-2-(1-(3-bromophenyl)ethyl)hydrazine-1-carboxylate (compound 7, Table 2). Prepared according to the general procedure utilizing benzyl (E)-2-(1-(3-bromophenyl)ethylidene)hydrazine-1carboxylate (347 mg, $1.00 \mathrm{mmol})$, bis(2-methallyl)(1,5cyclooctadiene)ruthenium(II) (3.2 mg, $1 \mathrm{~mol} \%$ ), Walphos ligand SL-W022$1(7.6 \mathrm{mg}, 1.1 \mathrm{~mol} \%), \mathrm{HBF}_{4}-\mathrm{OEt}_{2}(2.7 \mu \mathrm{L}, 2 \mathrm{~mol} \%)$ and hydrogen (100 psi) to give the title product (95\%, 97\% ee). ${ }^{1} \mathrm{H}$ NMR (400 MHz, DMSO-d $): \delta 8.59(1 \mathrm{H}, \mathrm{s}), 7.54(1 \mathrm{H}, \mathrm{s}), 7.42(1 \mathrm{H}$, ddd (app. $\mathrm{dt}), J=7.8,1.2 \mathrm{~Hz}), 7.39-7.19(7 \mathrm{H}, \mathrm{m}), 5.11-4.86(3 \mathrm{H}, \mathrm{m}), 4.13(1 \mathrm{H}, \mathrm{s}), 1.17(3 \mathrm{H}, \mathrm{d}, J=6.6 \mathrm{~Hz})$. ${ }^{13} \mathrm{C}$ NMR (100 MHz, DMSO-d d : (1 overlapping signal) $\delta$ 156.9, 147.3, 137.1, 130.3, 129.7, 128.3, 127.8, 127.6, 126.3, 121.6, 65.2, 57.8, 21.7. HRMS (ESI) m/z: $[M+H]^{+}$Calculated for $\mathrm{C}_{16} \mathrm{H}_{18} \mathrm{~N}_{2} \mathrm{O}_{2} \mathrm{Br} 349.0552$; found 349.0551 .

Chiral SFC analysis of 7 (Chiralpak IA, $35^{\circ} \mathrm{C}, 100$ bar, cosolvent: $20 \mathrm{mM} \mathrm{NH}_{3}$ in $\mathrm{MeOH}, 4 \mathrm{~mL} / \mathrm{min}$, $0 \mathrm{~min}$ to $4 \mathrm{~min}$ ramp $10-50 \%$ cosolvent then hold $50 \%$ cosolvent for $2 \mathrm{~min}$ )

Racemate:

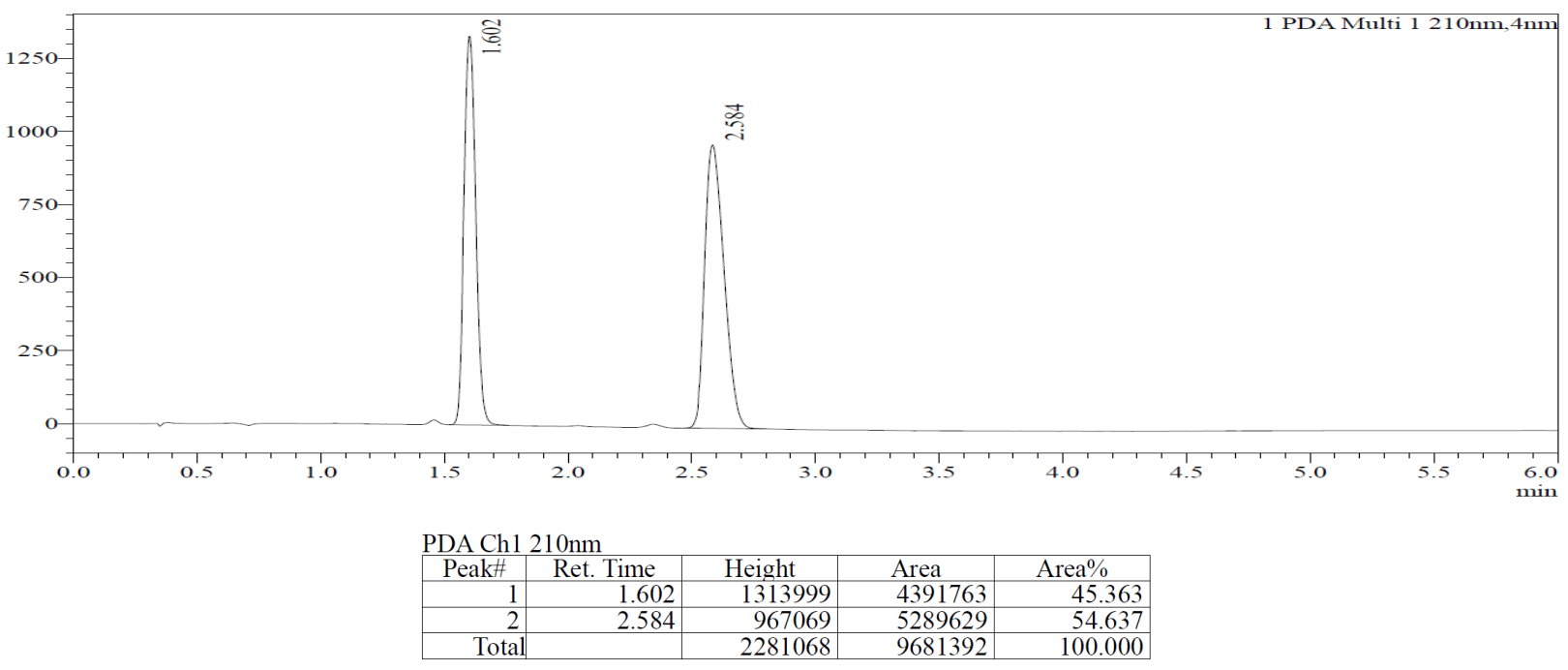

Reaction Product:

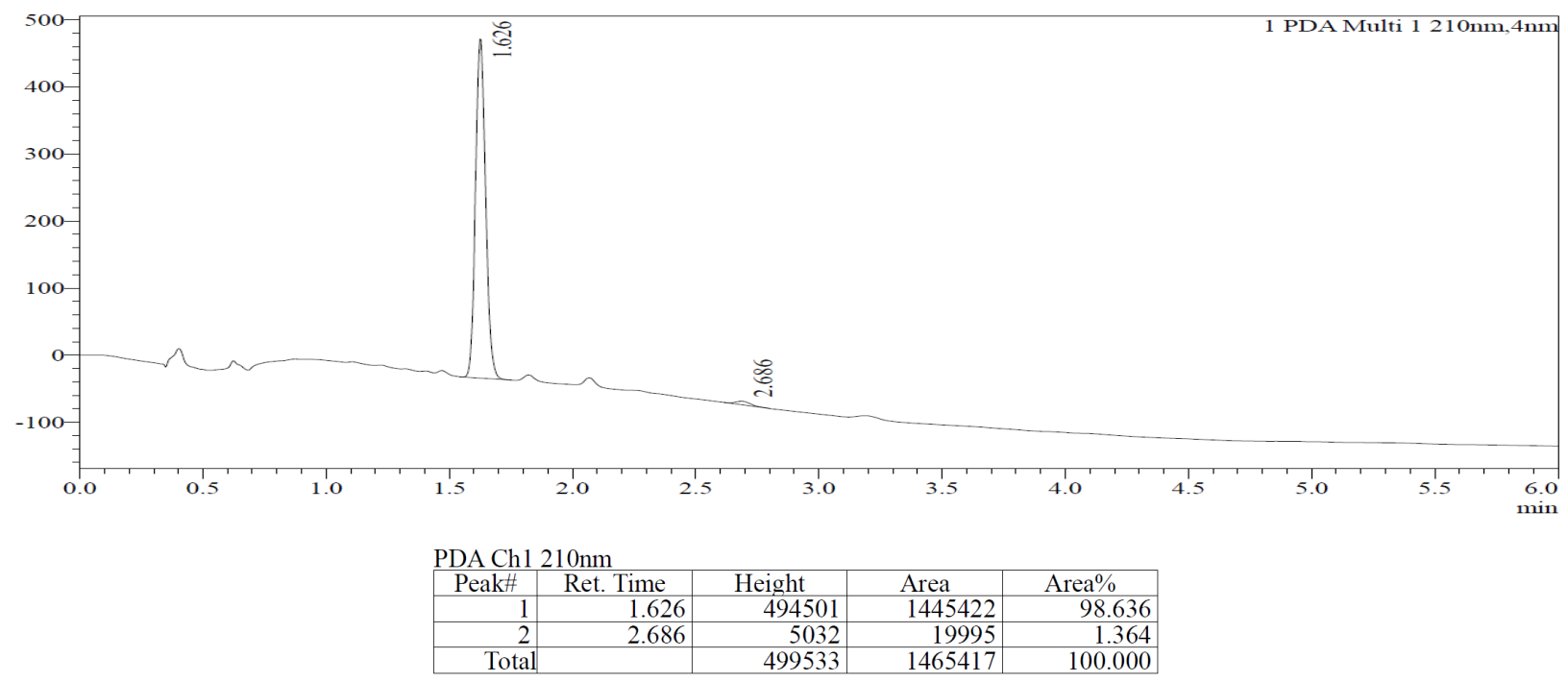




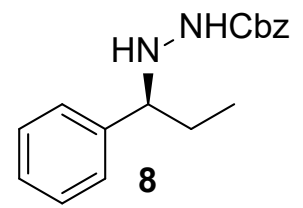

benzyl (S)-2-(1-phenylpropyl)hydrazine-1-carboxylate (compound 8, Table 2). Prepared according to the general procedure utilizing benzyl $(E)-2-$ (1-phenylpropylidene)hydrazine-1-carboxylate $(282 \mathrm{mg}, 1.00 \mathrm{mmol})$, bis $(2-$ methallyl)(1,5-cyclooctadiene)ruthenium(II) $(3.2 \mathrm{mg}, 1 \mathrm{~mol} \%)$, Walphos ligand SL-W022-1 (7.6 mg, 1.1 mol\%), $\mathrm{HBF}_{4}-\mathrm{OEt}_{2}(2.7 \mu \mathrm{L}, 2 \mathrm{~mol} \%)$ and hydrogen (100 psi) to give the title product $\left(92 \%, 96 \%\right.$ ee). ${ }^{1} \mathrm{H}$ NMR $\left(400 \mathrm{MHz}, \mathrm{DMSO}-\mathrm{d}_{6}\right): \delta 8.52(1 \mathrm{H}, \mathrm{s})$, 7.46-7.17 (10H, m), $5.01(2 \mathrm{H}, \mathrm{s}), 4.77(1 \mathrm{H}, \mathrm{s}), 3.90(1 \mathrm{H}, \mathrm{s}), 1.69(1 \mathrm{H}$, ddq (app dpent.), J = 13.3, $7.2 \mathrm{~Hz}), 1.45(1 \mathrm{H}$, ddq (app dpent), J = 13.6, $7.4 \mathrm{~Hz}), 0.70(3 \mathrm{H}, \mathrm{dd}(\operatorname{app~t}), \mathrm{J}=7.3 \mathrm{~Hz}) .{ }^{13} \mathrm{C} \mathrm{NMR}$ (100 MHz, DMSO-d $\mathrm{d}_{6}$ ): (1 overlapping signal) $\delta 156.9,142.4,137.1,128.3,128.0,127.8,127.6$, 126.9, 65.2, 64.9, 27.8, 10.1. HRMS (ESI) $\mathrm{m} / \mathrm{z}$ : $[\mathrm{M}+\mathrm{H}]^{+}$Calculated for $\mathrm{C}_{17} \mathrm{H}_{21} \mathrm{~N}_{2} \mathrm{O}_{2} 285.1603$; found 285.1604 .

Chiral SFC analysis of 8 (Chiralpak IA, $35^{\circ} \mathrm{C}, 100$ bar, cosolvent: $20 \mathrm{mM} \mathrm{NH}_{3}$ in $\mathrm{MeOH}, 4 \mathrm{~mL} / \mathrm{min}$, $0 \mathrm{~min}$ to $4 \mathrm{~min}$ ramp $10-50 \%$ cosolvent then hold $50 \%$ cosolvent for $2 \mathrm{~min}$ )

Racemate:
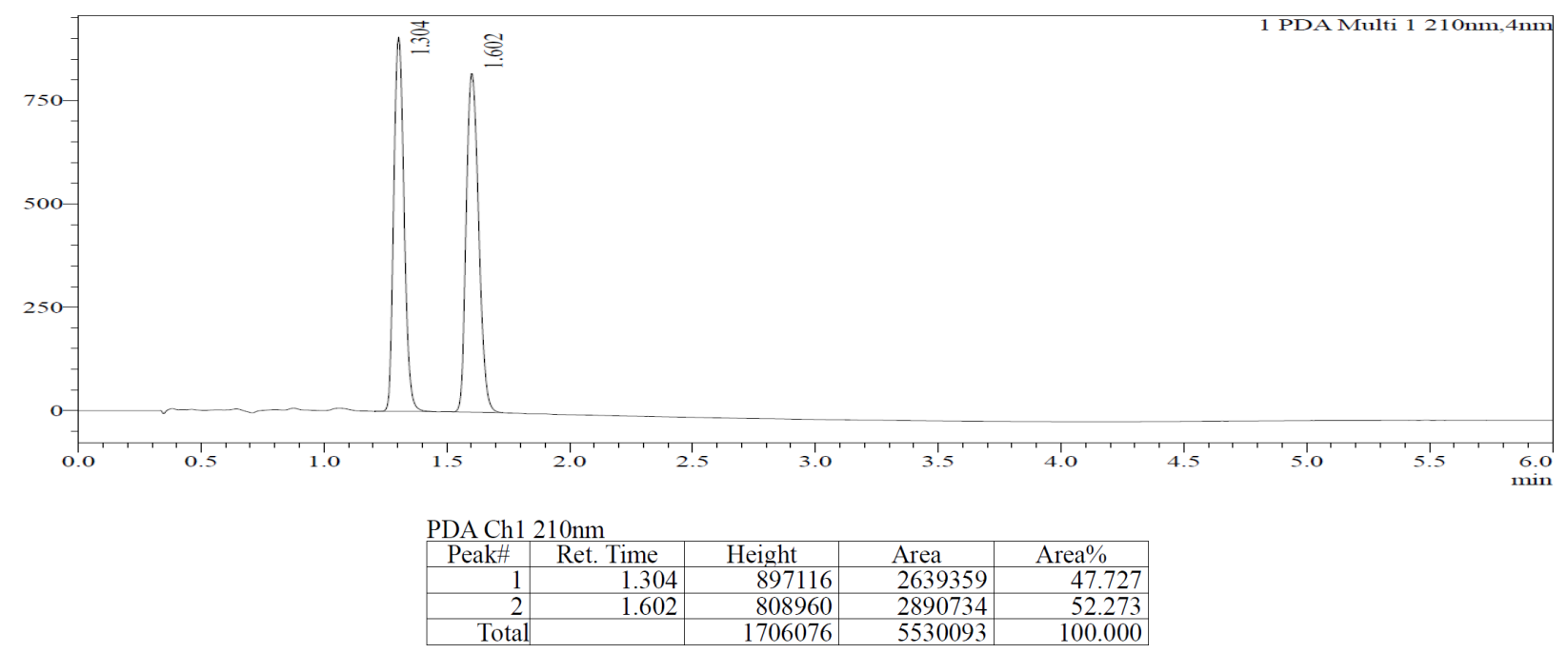

Reaction Product:
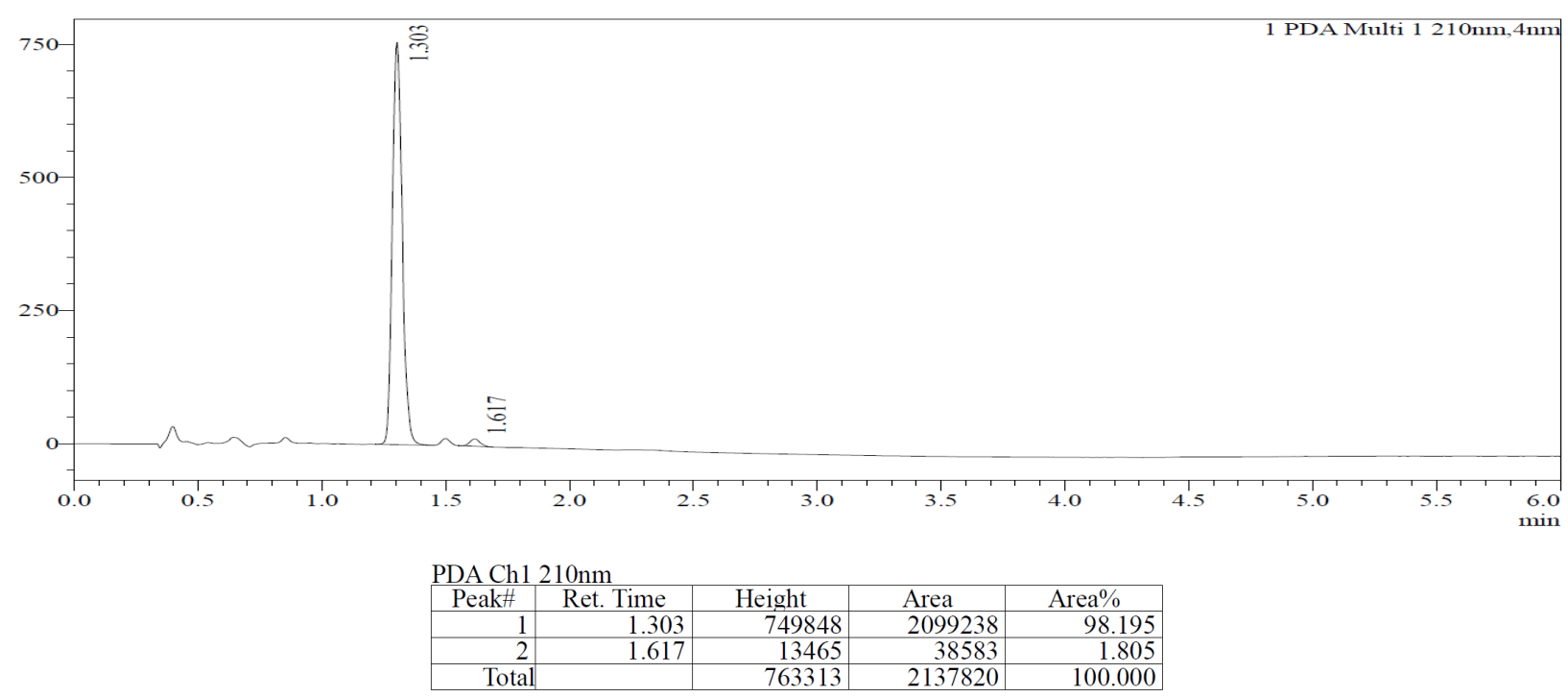
<smiles>CC(=O)NNC(c1ccccc1)C1CCCCC1</smiles>

(S)-2-(cyclohexyl(phenyl)methyl)hydrazine-1-carboxylate (compound 9, Table 2). Prepared according to the general procedure utilizing benzyl (E)-2-(cyclohexyl(phenyl)methylene)hydrazine-1-carboxylate (336 mg, $1.00 \mathrm{mmol}, \quad 93: 7$ mixture of $E: Z$ isomers), bis(2-methallyl)(1,5cyclooctadiene)ruthenium(II) (3.2 mg, 1 mol\%), Walphos ligand SL-W022-1 (7.6 mg, $1.1 \mathrm{~mol} \%), \mathrm{HBF}_{4}-\mathrm{OEt}_{2}(2.7 \mu \mathrm{L}, 2 \mathrm{~mol} \%)$ and hydrogen (200 psi) to give the title product (95\%, 87\% ee). ${ }^{1} \mathrm{H}$ NMR (400 MHz, DMSO-d 6 ): $\delta 8.43(1 \mathrm{H}, \mathrm{s}), 7.48-7.12(10 \mathrm{H}, \mathrm{m}), 4.99(2 \mathrm{H}, \mathrm{s})$, $4.75(1 \mathrm{H}, \mathrm{s}), 3.84(1 \mathrm{H}, \mathrm{s}), 1.18(1 \mathrm{H}, \mathrm{d}, \mathrm{J}=11.8 \mathrm{~Hz}), 1.72-1.34(5 \mathrm{H}, \mathrm{m}), 1.26-0.60(5 \mathrm{H}, \mathrm{m}) .{ }^{13} \mathrm{C}$ NMR (100 MHz, DMSO-d $\left.\mathrm{d}_{6}\right): \delta 156.8,141.1,137.1,128.4,128.3,127.7,127.6,127.5,126.7,68.4$, 65.1, 41.4, 29.6, 28.3, 26.1, 25.9, 25.7. HRMS (ESI) m/z: $[\mathrm{M}+\mathrm{H}]^{+}$Calculated for $\mathrm{C}_{21} \mathrm{H}_{27} \mathrm{~N}_{2} \mathrm{O}_{2}$ 339.2073; found 339.2071.

Chiral SFC analysis of 9 (Chiralcel OD, $35^{\circ} \mathrm{C}, 100$ bar, cosolvent: $20 \mathrm{mM} \mathrm{NH}_{3}$ in $\mathrm{MeOH}, 4 \mathrm{~mL} / \mathrm{min}$, 0 min to 3 min ramp $5-20 \%$ cosolvent then hold $20 \%$ cosolvent for $1 \mathrm{~min}$ )

Racemate:

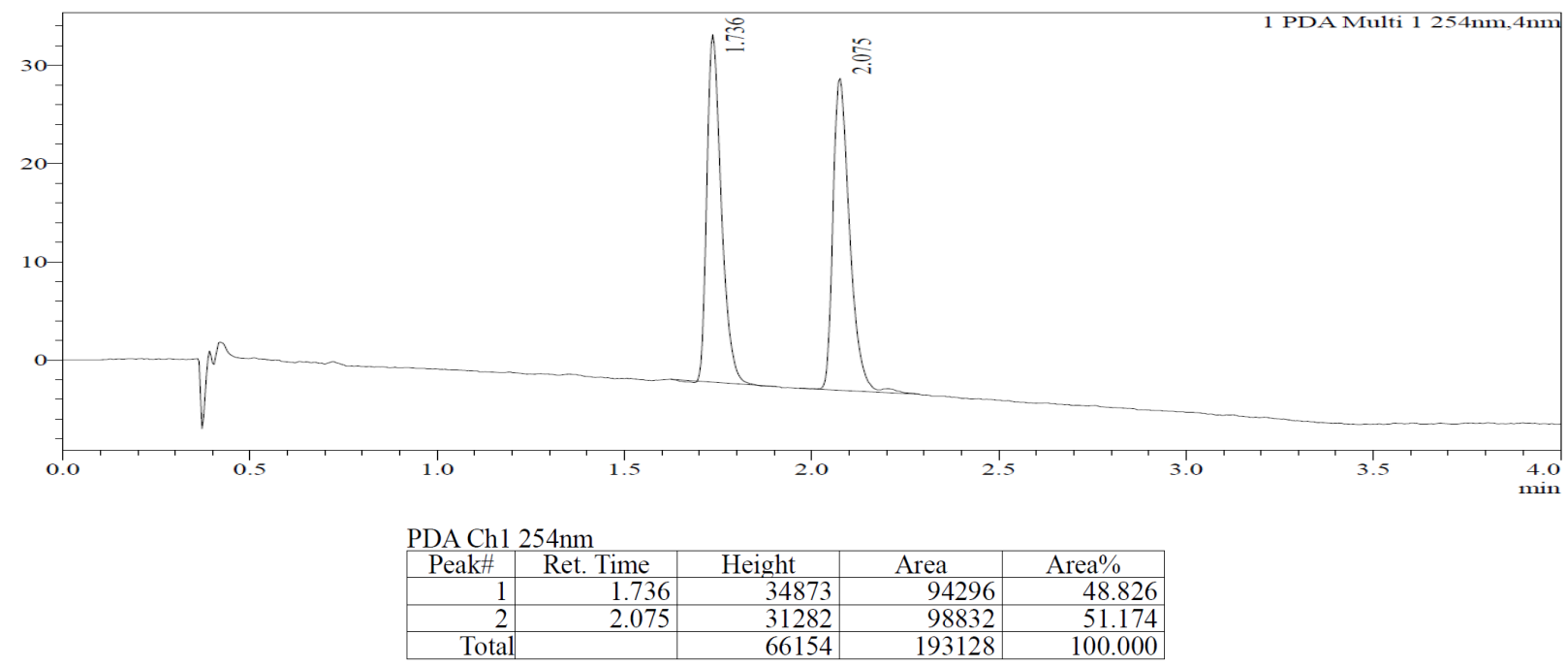

Reaction Product (93:7, E:Z SM)

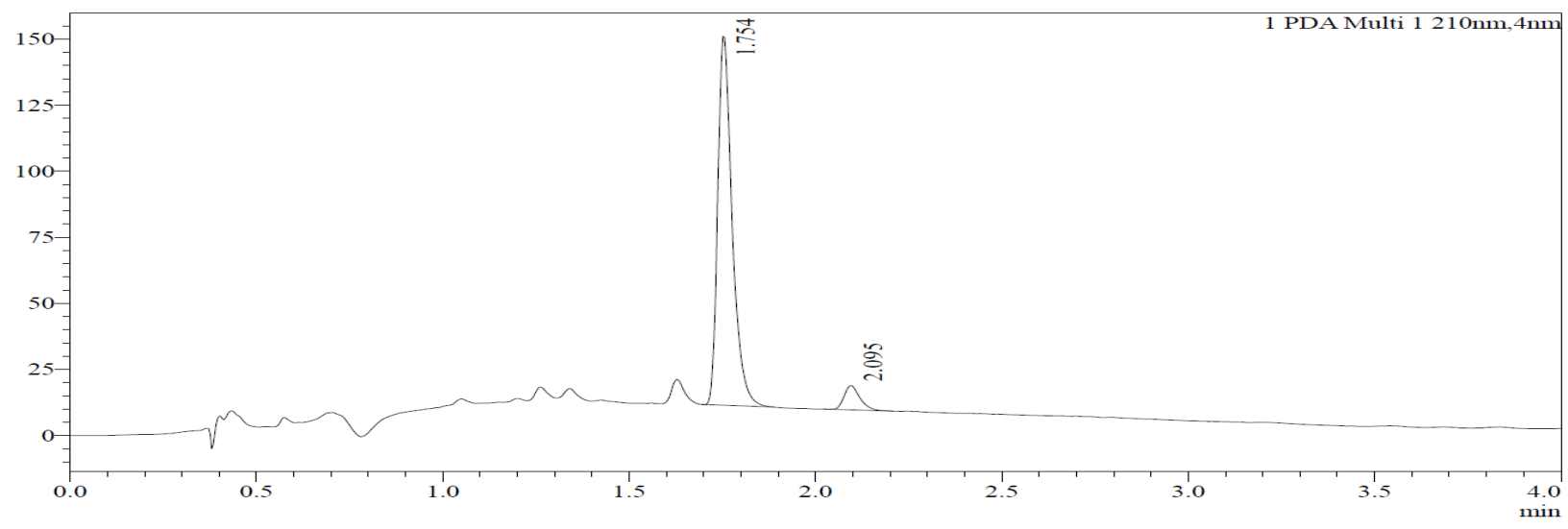

PDA Ch1 210nm
\begin{tabular}{|r|r|r|r|r|}
\hline Peak\# & Ret. Time & \multicolumn{1}{c|}{ Height } & \multicolumn{1}{c|}{ Area } & \multicolumn{1}{|c|}{ Area $\%$} \\
\hline 1 & 1.754 & 135504 & 376929 & 93.343 \\
\hline 2 & 2.095 & 9145 & 26882 & 6.657 \\
\hline Total & & 144648 & 403811 & 100.000 \\
\hline
\end{tabular}


Chiral SFC analysis of 9 (Chiralcel OD, $35^{\circ} \mathrm{C}, 100$ bar, cosolvent: $0.1 \%$ DEA in $\mathrm{MeOH}, 4$ $\mathrm{mL} / \mathrm{min}, 0$ min to $2 \mathrm{~min}$ ramp $5-20 \%$ cosolvent then hold $20 \%$ cosolvent for $1 \mathrm{~min}$ )

Racemate:

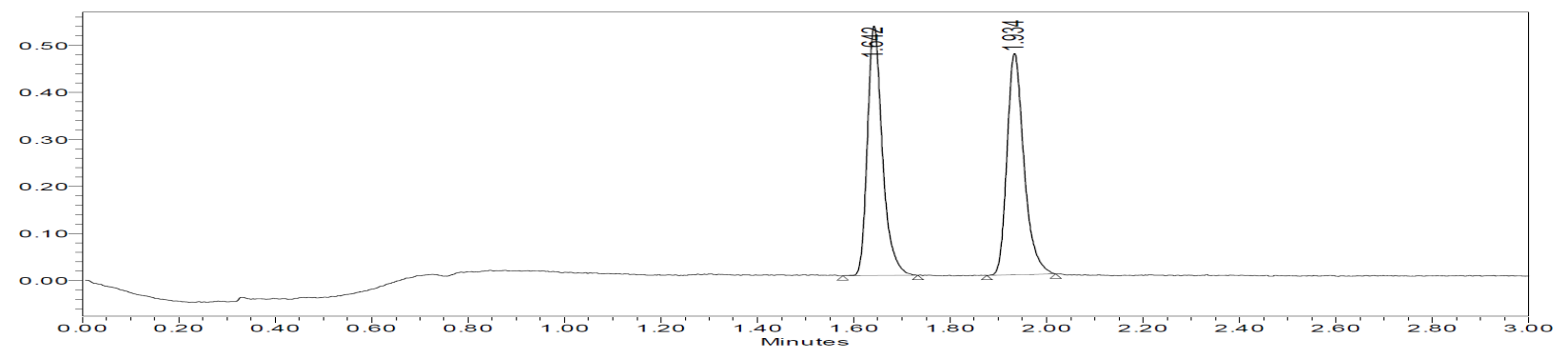

\begin{tabular}{|c|c|c|r|}
\hline & RT & Area & $\%$ Area \\
\hline 1 & 1.642 & 1147083 & 49.85 \\
\hline 2 & 1.934 & 1154152 & 50.15 \\
\hline
\end{tabular}

Reaction Product (pure E SM):

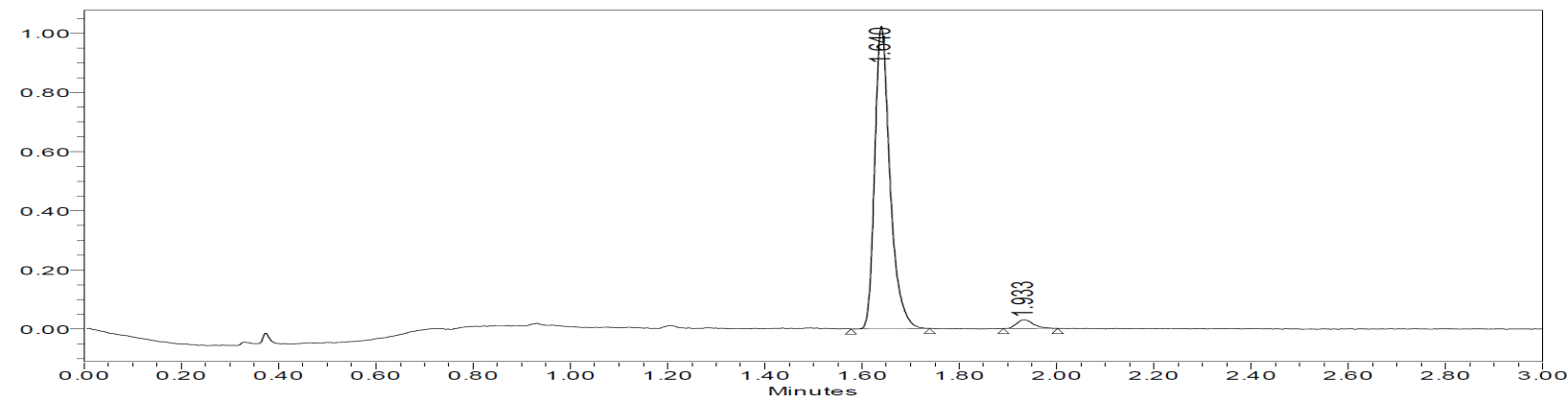

\begin{tabular}{|r|c|r|r|}
\hline & RT & Area & $\%$ Area \\
\hline 1 & 1.640 & 2236443 & 96.96 \\
\hline 2 & 1.933 & 70139 & 3.04 \\
\hline
\end{tabular}

Reaction Product (pure Z SM):

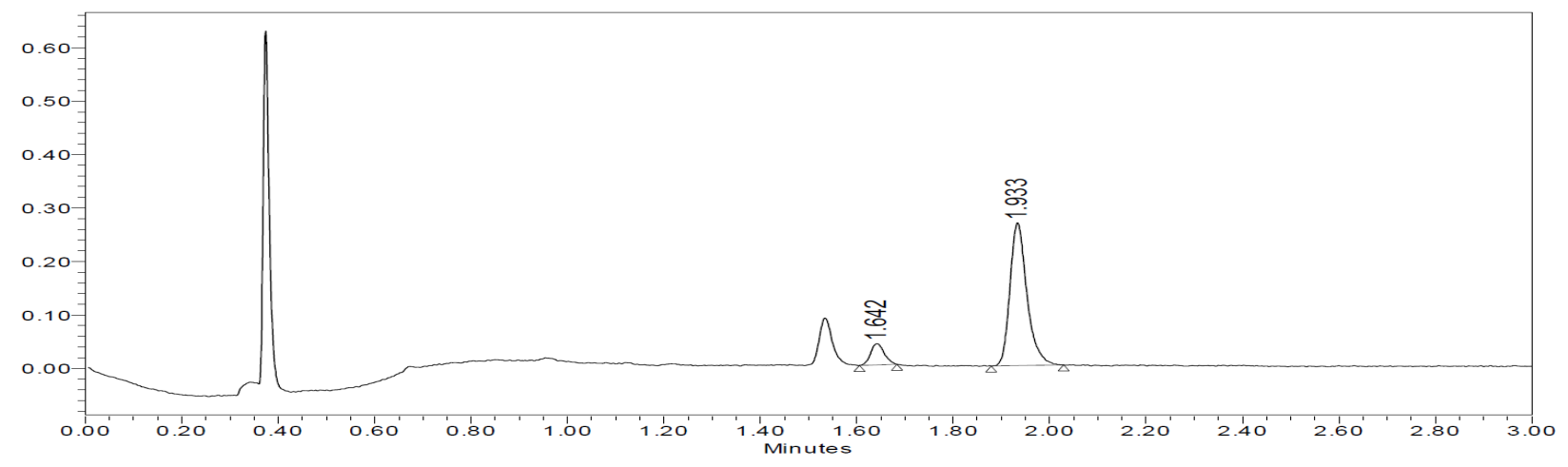

\begin{tabular}{|c|c|c|r|}
\hline & RT & Area & \% Area \\
\hline 1 & 1.642 & 77540 & 10.60 \\
\hline 2 & 1.933 & 653634 & 89.40 \\
\hline
\end{tabular}



Table 2). Prepared according to the general procedure utilizing benzyl $(E)-2-$ (1-cyclohexylethylidene)hydrazine-1-carboxylate $(274 \mathrm{mg}, 1.00 \mathrm{mmol})$, bis(2methallyl)(1,5-cyclooctadiene)ruthenium(II) (3.2 mg, $1 \mathrm{~mol} \%$ ), Walphos ligand SL-W022-1 (7.6 mg, 1.1 mol\%), $\mathrm{HBF}_{4}-\mathrm{OEt}_{2}(2.7 \mu \mathrm{L}, 2 \mathrm{~mol} \%)$ and hydrogen (500 psi) to give the title product $\left(86 \%, 10 \%\right.$ ee). ${ }^{1} \mathrm{H}$ NMR $\left(400 \mathrm{MHz}, \mathrm{DMSO}-\mathrm{d}_{6}\right): \delta 8.43(1 \mathrm{H}, \mathrm{s})$, 7.48-7.12 $(10 \mathrm{H}, \mathrm{m}), 4.99(2 \mathrm{H}, \mathrm{s}), 4.75(1 \mathrm{H}, \mathrm{s}), 3.84(1 \mathrm{H}, \mathrm{s}), 1.18(1 \mathrm{H}, \mathrm{d}, \mathrm{J}=11.8 \mathrm{~Hz}), 1.72-1.34$ $(5 \mathrm{H}, \mathrm{m}), 1.26-0.60(5 \mathrm{H}, \mathrm{m}) .{ }^{13} \mathrm{C}$ NMR $\left(100 \mathrm{MHz}, \mathrm{DMSO}-\mathrm{d}_{6}\right): \delta 156.8,141.1,137.1,128.4,128.3$, 127.7, 127.6, 127.5, 126.7, 68.4, 65.1, 41.4, 29.6, 28.3, 26.1, 25.9, 25.7. HRMS (ESI) m/z: [M+H] ${ }^{+}$ Calculated for $\mathrm{C}_{16} \mathrm{H}_{25} \mathrm{~N}_{2} \mathrm{O}_{2}$ 277.1916; found 277.1917.

Chiral SFC analysis of 10 (Chiralpak ID, $35^{\circ} \mathrm{C}, 100$ bar, cosolvent: $20 \mathrm{mM} \mathrm{NH}_{3}$ in $\mathrm{MeOH}, 2$ $\mathrm{mL} / \mathrm{min}, 0 \mathrm{~min}$ to $4 \mathrm{~min}$ ramp $10-50 \%$ cosolvent then hold $50 \%$ cosolvent for $2 \mathrm{~min}$ )

Racemate:

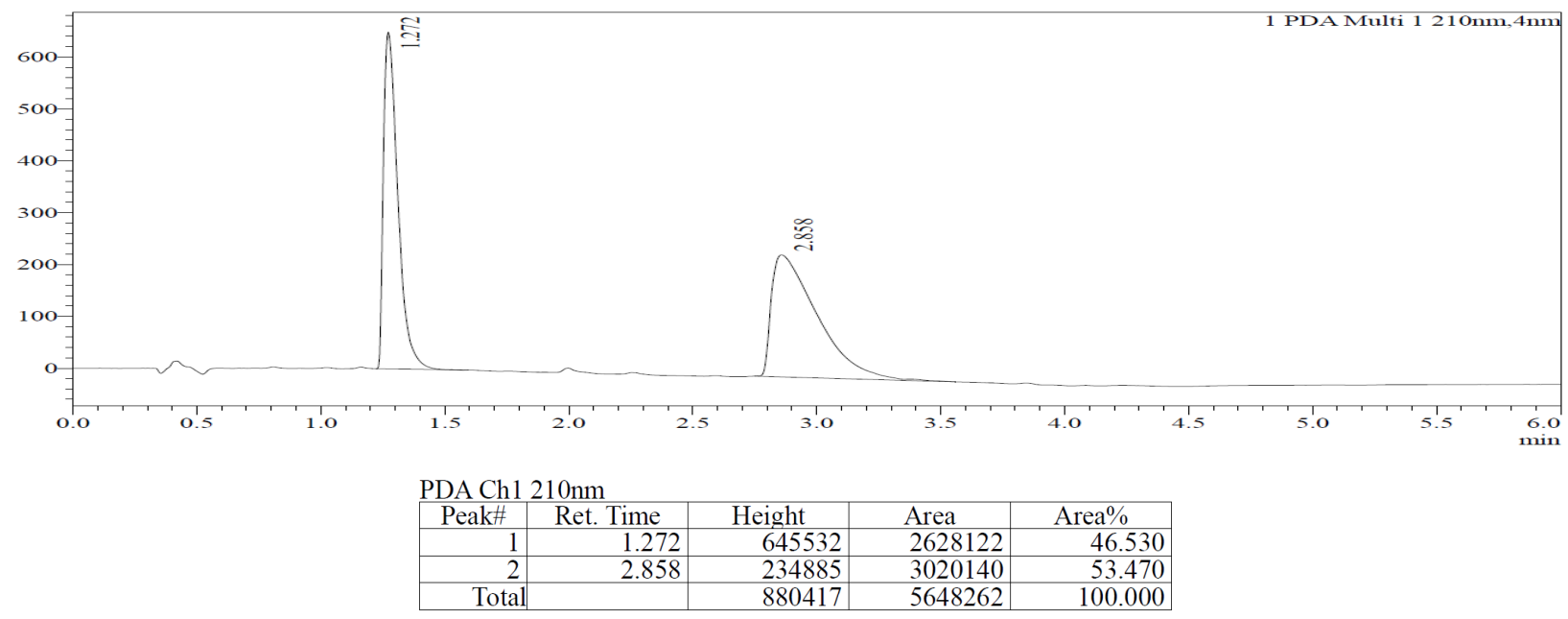

Reaction Product:

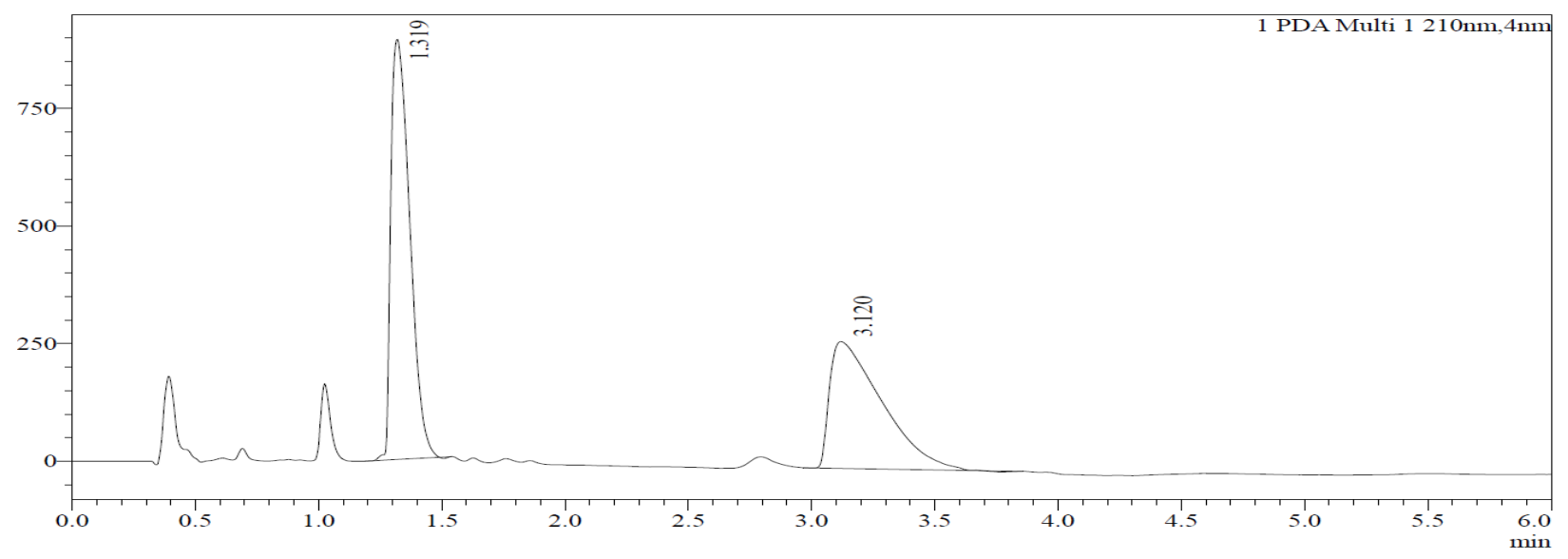

PDA Ch1 $210 \mathrm{~nm}$
\begin{tabular}{|r|r|r|r|r|}
\hline Peak\# & Ret. Time & Height & Area & \multicolumn{1}{|c|}{ Area $\%$} \\
\hline 1 & 1.319 & 892716 & 4784725 & 54.731 \\
\hline 2 & 3.120 & 269381 & 3957592 & 45.269 \\
\hline Total & & 1162097 & 8742318 & 100.000 \\
\hline
\end{tabular}


<smiles>O=C(NNC(c1ccccc1)C(F)(F)F)c1ccccc1</smiles>

(R)-2-(2,2,2-trifluoro-1-phenylethyl)hydrazine-1-carboxylate (compound 11, Table 2). Prepared according to the general procedure utilizing benzyl (Z)-2-(2,2,2-trifluoro-1-phenylethylidene)hydrazine-1carboxylate $\quad(322 \quad \mathrm{mg}, \quad 1.00 \quad \mathrm{mmol}), \quad$ bis $(2-$ methallyl $)(1,5-$ cyclooctadiene)ruthenium(II) (3.2 mg, $1 \mathrm{~mol} \%$ ), Walphos ligand SL-W022-1 (7.6 mg, $1.1 \mathrm{~mol} \%), \mathrm{HBF}_{4}-\mathrm{OEt}_{2}(2.7 \mu \mathrm{L}, 2 \mathrm{~mol} \%)$ and hydrogen (500 psi) to give the title product $\left(18 \%, 43 \%\right.$ ee). ${ }^{1} \mathrm{H}$ NMR $\left(400 \mathrm{MHz}, \mathrm{MeOD}-\mathrm{d}_{4}\right): \delta 7.63-7.13(10 \mathrm{H}, \mathrm{m}), 5.08(2 \mathrm{H}, \mathrm{s}), 4.89(2 \mathrm{H}$ (assumed, overlaps with residual water), s), $4.66(1 \mathrm{H}$, ddd (app q), $J=7.2 \mathrm{~Hz}) .{ }^{13} \mathrm{C}$ NMR $(100$ $\left.\mathrm{MHz}, \mathrm{MeOD}-\mathrm{d}_{4}\right): \delta 159.3,138.0,133.9,132.2,130.3,129.6,129.5,129.0,128.7,126.5\left(\mathrm{q}, J_{C-F}=\right.$ $280.9 \mathrm{~Hz}), 67.6,66.3\left(\mathrm{q}, \mathrm{J}_{\mathrm{C}-\mathrm{F}}=27.0 \mathrm{~Hz}\right)$. HRMS $(\mathrm{ESI}) \mathrm{m} / \mathrm{z}$ : $[\mathrm{M}+\mathrm{H}]^{+}$Calculated for $\mathrm{C}_{16} \mathrm{H}_{16} \mathrm{~N}_{2} \mathrm{O}_{2} \mathrm{~F}_{3}$ 325.1164; found 325.1164.

Chiral SFC analysis of 11 (Chiralpak IA, $35{ }^{\circ} \mathrm{C}, 100$ bar, cosolvent: $20 \mathrm{mM} \mathrm{NH}_{3}$ in $\mathrm{MeOH}, 4$ $\mathrm{mL} / \mathrm{min}, 0 \mathrm{~min}$ to $3 \mathrm{~min}$ ramp $5-20 \%$ cosolvent then hold $20 \%$ cosolvent for $1 \mathrm{~min}$ )

Racemate:

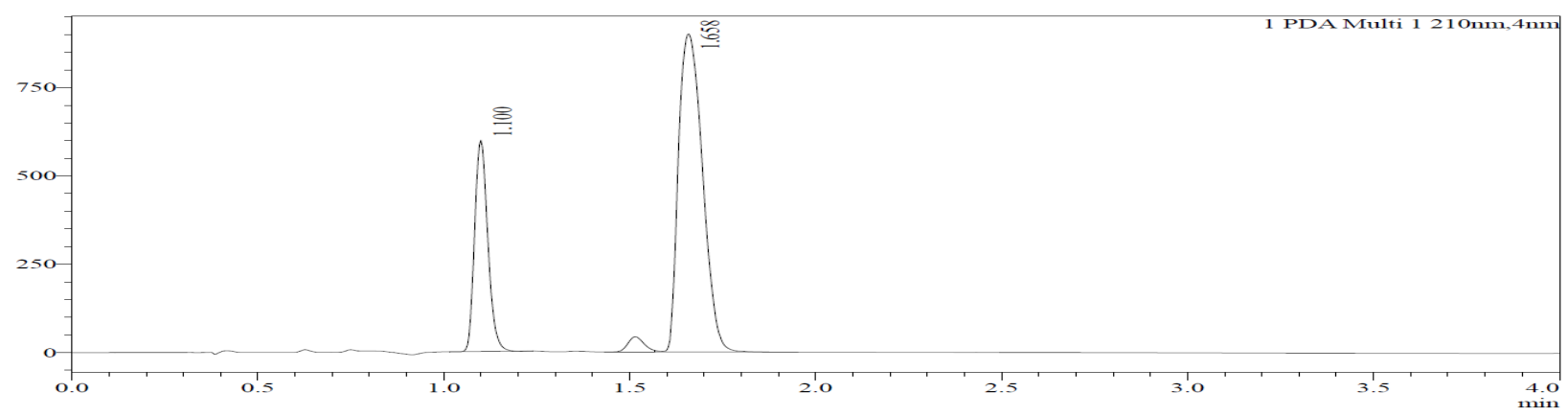

PDA Ch1 $210 \mathrm{~nm}$
\begin{tabular}{|r|r|r|r|r|}
\hline Peak\# & Ret. Time & Height & Area & \multicolumn{1}{|c|}{ Area $\%$} \\
\hline 1 & 1.100 & 587310 & 1475807 & 26.731 \\
\hline 2 & 1.658 & 892344 & 4045144 & 73.269 \\
\hline Total & & 1479654 & 5520951 & 100.000 \\
\hline
\end{tabular}

Reaction Product:

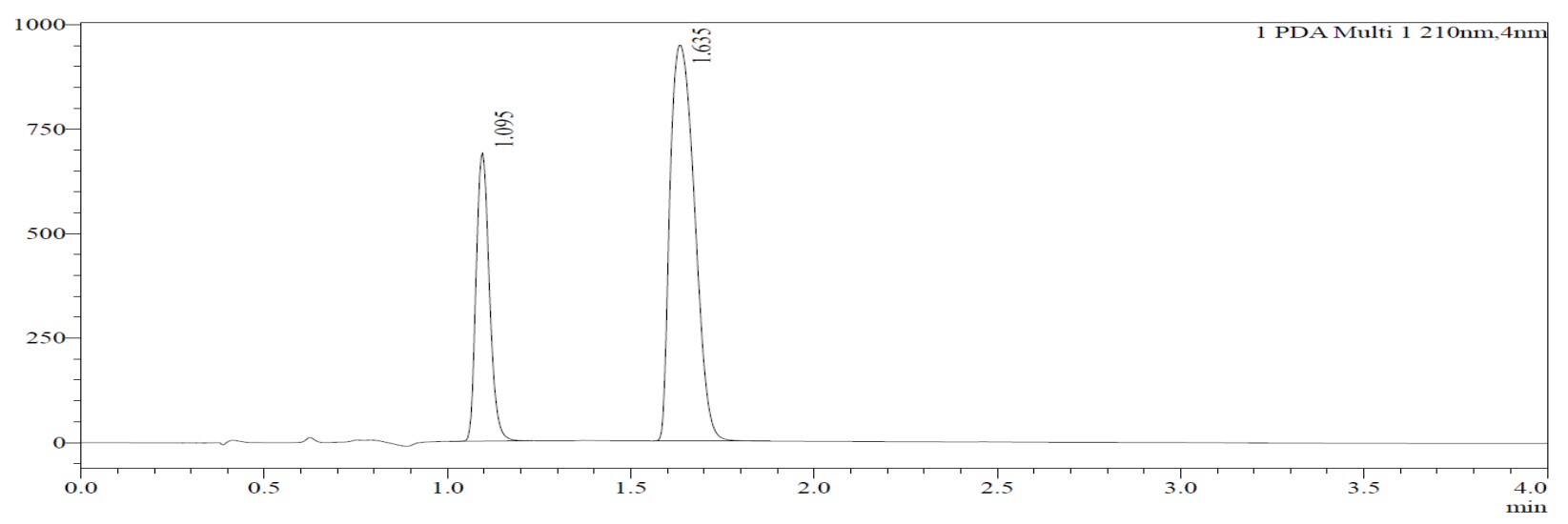

PDA Ch1 $210 \mathrm{~nm}$
\begin{tabular}{|r|r|r|r|r|}
\hline Peak\# & Ret. Time & Height & Area & Area $\%$ \\
\hline 1 & 1.095 & 684999 & 1739969 & 28.283 \\
\hline 2 & 1.635 & 942969 & 4411971 & 71.717 \\
\hline Total & & 1627968 & 6151941 & 100.000 \\
\hline
\end{tabular}




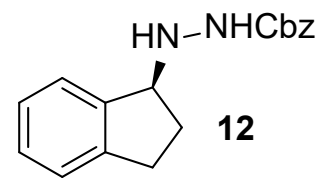

benzyl

(S)-2-(2,3-dihydro-1H-inden-1-yl)hydrazine-1-carboxylate (compound 12, Table 2). Prepared according to the general procedure utilizing benzyl carboxylate $\quad(280$

(E)-2-(2,3-dihydro-1H-inden-1-ylidene)hydrazine-1$\mathrm{mg}, \quad 1.00 \quad \mathrm{mmol}), \quad$ bis(2-methallyl $)(1,5-$ cyclooctadiene)ruthenium(II) (3.2 mg, $1 \mathrm{~mol} \%$ ), Walphos ligand SL-W022-1 (7.6 mg, $1.1 \mathrm{~mol} \%$ ), $\mathrm{HBF}_{4}-\mathrm{OEt}_{2}(2.7 \mu \mathrm{L}, 2 \mathrm{~mol} \%)$ and hydrogen (500 psi) to give the title product $\left(75 \%, 93 \%\right.$ ee). ${ }^{1} \mathrm{H}$ NMR $\left(400 \mathrm{MHz}, \mathrm{DMSO}-\mathrm{d}_{6}\right): \delta 8.71(1 \mathrm{H}, \mathrm{s}), 7.44-7.27(6 \mathrm{H}, \mathrm{m}), 7.25-7.09(3 \mathrm{H}, \mathrm{m}), 5.12-5.02(2 \mathrm{H}$, m), $4.79(1 \mathrm{H}, \mathrm{s}), 4.43(1 \mathrm{H}, \mathrm{s}), 2.92(1 \mathrm{H}$, ddd (app dt), $J=15.4,7.5 \mathrm{~Hz}), 2.73(1 \mathrm{H}$, ddd, $J=15.4$, 8.2, $5.4 \mathrm{~Hz}$ ), 2.08 (1H, dddd (app dq), $J=13.5,6.9 \mathrm{~Hz}$ ), 1.91 (1H, dddd (app ddt), $J=13.0,8.5$, $4.9 \mathrm{~Hz}$ ). ${ }^{13} \mathrm{C} \mathrm{NMR}(100 \mathrm{MHz}$, DMSO-d 6 ): $\delta 157.1,144.1,143.1,137.1,128.4,127.8,127.7,127.5$, 125.9, 124.8, 124.4, 65.4, 64.0, 30.7, 29.9. HRMS (ESI) m/z: $[\mathrm{M}+\mathrm{H}]^{+}$Calculated for $\mathrm{C}_{17} \mathrm{H}_{19} \mathrm{~N}_{2} \mathrm{O}_{2}$ 283.1447; found 283.1447.

Chiral SFC analysis of 12 (Chiralcel OD, $35^{\circ} \mathrm{C}, 100$ bar, cosolvent: $0.1 \%$ DEA in $\mathrm{MeOH}, 4 \mathrm{~mL} / \mathrm{min}$, 0 min to 2 min ramp $5-20 \%$ cosolvent then hold $20 \%$ cosolvent for $1 \mathrm{~min}$ )

Racemate:

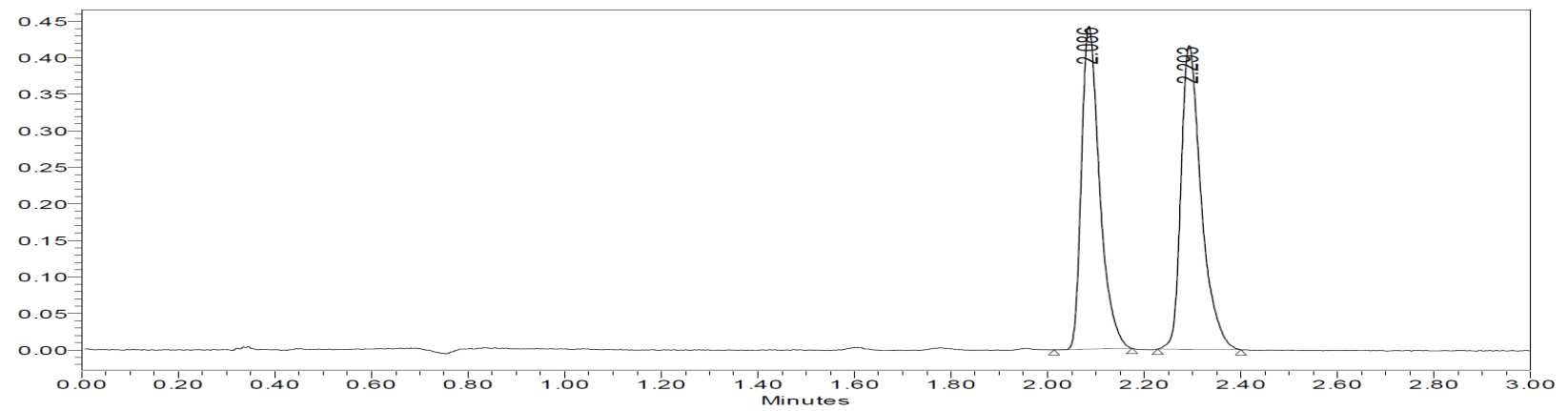

\begin{tabular}{|c|c|c|r|}
\hline & RT & Area & \% Area \\
\hline 1 & 2.086 & 1152934 & 48.78 \\
\hline 2 & 2.293 & 1210533 & 51.22 \\
\hline
\end{tabular}

Reaction Product:

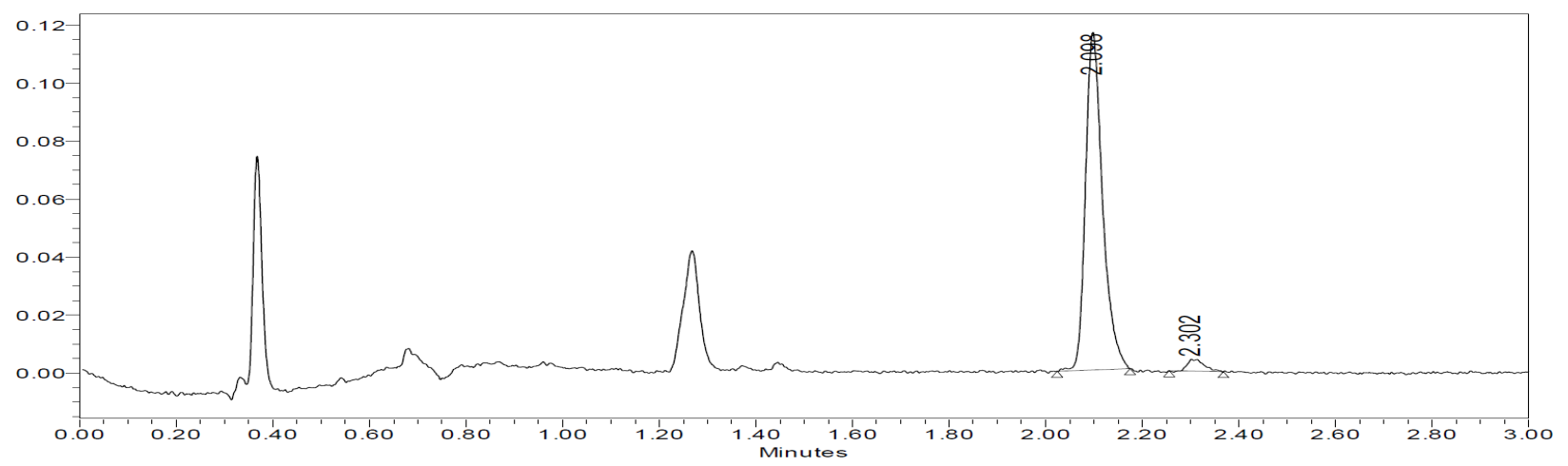

\begin{tabular}{|r|c|r|r|}
\hline & RT & Area & \% Area \\
\hline 1 & 2.098 & 286352 & 96.61 \\
\hline 2 & 2.302 & 10048 & 3.39 \\
\hline
\end{tabular}


<smiles>CC(C)(C)NNC1Cc2ccccc21</smiles>

benzyl

(S)-2-(bicyclo[4.2.0]octa-1,3,5-trien-7-yl)hydrazine-1carboxylate (compound 13, Table 2). Prepared according to the general procedure utilizing benzyl (E)-2-(bicyclo[4.2.0]octa-1,3,5-trien-7ylidene)hydrazine-1-carboxylate (266 mg, $1.00 \mathrm{mmol})$, bis(2-methallyl)(1,5cyclooctadiene)ruthenium(II) (3.2 mg, $1 \mathrm{~mol} \%)$, Walphos ligand SL-W022-1 (7.6 mg, $1.1 \mathrm{~mol} \%$ ), $\mathrm{HBF}_{4}-\mathrm{OEt}_{2}(2.7 \mu \mathrm{L}, 2 \mathrm{~mol} \%)$ and hydrogen (100 psi) to give the title product $(61 \%,>99 \%$ ee $) .{ }^{1} \mathrm{H}$ NMR (400 MHz, DMSO-d $)$ : $\delta 8.75(1 \mathrm{H}, \mathrm{s}), 7.46-7.05(9 \mathrm{H}, \mathrm{m}), 5.26(1 \mathrm{H}$, dd (app t), J = $4.4 \mathrm{~Hz})$, 5.12-4.98 $(2 \mathrm{H}, \mathrm{m}), 4.56(1 \mathrm{H}, \mathrm{s}), 3.26(1 \mathrm{H}, \mathrm{dd}, J=14.1,4.6 \mathrm{~Hz}), 3.00(1 \mathrm{H}, \mathrm{d}, J=14.1 \mathrm{~Hz}) .{ }^{13} \mathrm{C}$ NMR $\left(100 \mathrm{MHz}, \mathrm{DMSO}-\mathrm{d}_{6}\right): \delta 157.2,146.2,143.0,137.1,128.3,128.3,127.8,127.7,126.5$, 123.0, 122.9, 65.3, 60.5, 36.6. HRMS (ESI) m/z: $[\mathrm{M}+\mathrm{H}]^{+}$Calculated for $\mathrm{C}_{16} \mathrm{H}_{17} \mathrm{~N}_{2} \mathrm{O}_{2} 269.1290$; found 269.1283 .

Chiral SFC analysis of 13 (Lux Cellulose-3, $35^{\circ} \mathrm{C}, 100$ bar, cosolvent: $20 \mathrm{mM} \mathrm{NH}_{3}$ in $\mathrm{MeOH}, 4$ $\mathrm{mL} / \mathrm{min}, 0 \mathrm{~min}$ to $3 \mathrm{~min}$ ramp $5-20 \%$ cosolvent then hold $20 \%$ cosolvent for $1 \mathrm{~min}$ )

Racemate:

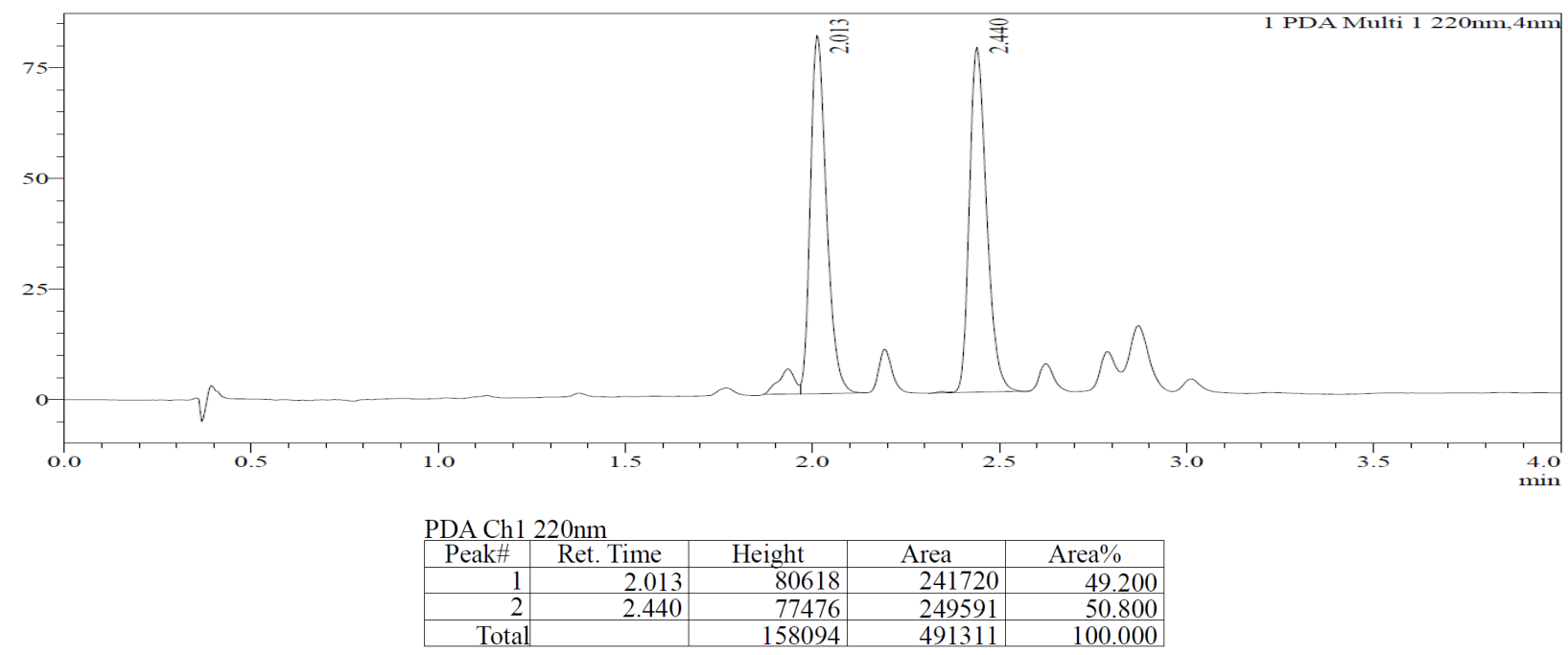

Reaction Product:

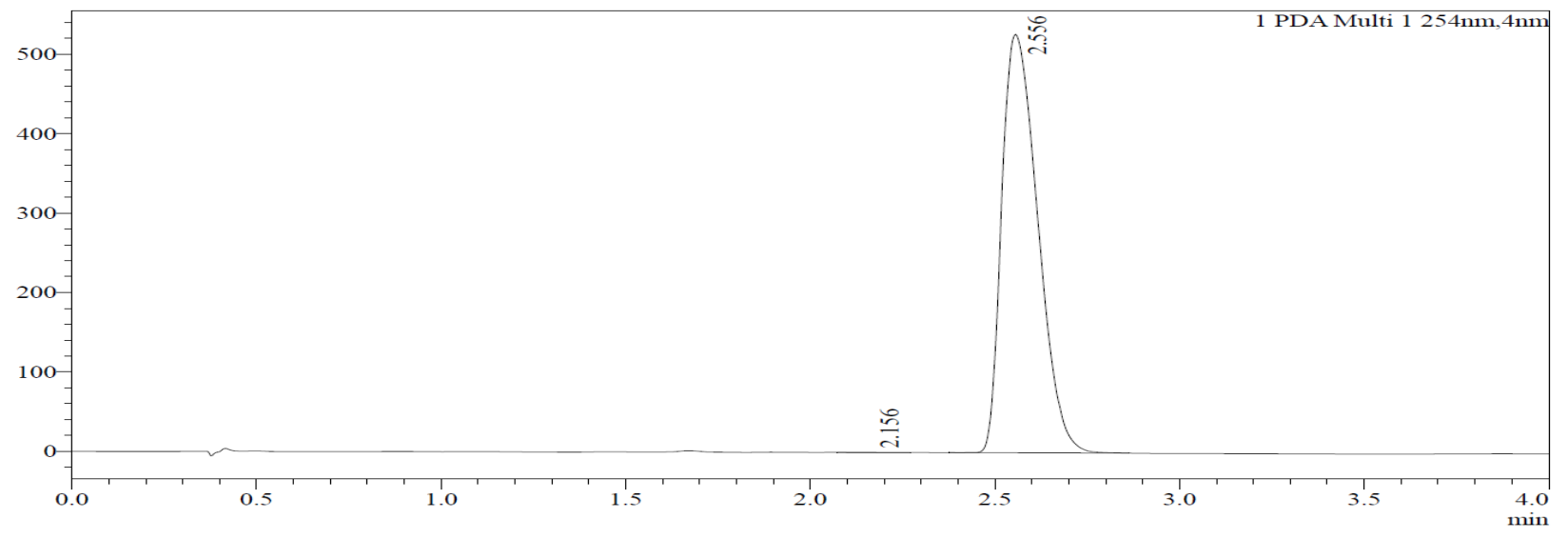

PDA Ch1 $254 \mathrm{~nm}$
\begin{tabular}{|r|r|r|r|r|}
\hline Peak\# & Ret. Time & Height & Area & Area $\%$ \\
\hline 1 & 2.156 & 580 & 3255 & 0.091 \\
\hline 2 & 2.556 & 525705 & 3579625 & 99.909 \\
\hline Total & & 526285 & 3582880 & 100.000 \\
\hline
\end{tabular}




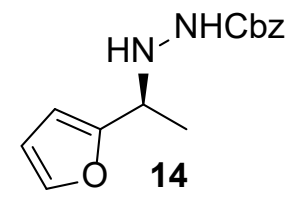

benzyl (S)-2-(1-(furan-2-yl)ethyl)hydrazine-1-carboxylate (compound 14, Table 2). Prepared according to the general procedure utilizing benzyl $(E)-2-$ (1-(furan-2-yl)ethylidene)hydrazine-1-carboxylate $(258 \mathrm{mg}, 1.00 \mathrm{mmol})$, bis(2methallyl)(1,5-cyclooctadiene)ruthenium(II) (3.2 mg, $1 \mathrm{~mol} \%)$, Walphos ligand SL-W022-1 (7.6 mg, $\left.1.1 \mathrm{~mol}^{2}\right)$, $\mathrm{HBF}_{4}-\mathrm{OEt}_{2}(2.7 \mu \mathrm{L}, 2 \mathrm{~mol} \%)$ and hydrogen (200 psi) to give the title product $\left(94 \%, 96 \%\right.$ ee). ${ }^{1} \mathrm{H}$ NMR $\left(400 \mathrm{MHz}, \mathrm{DMSO}-\mathrm{d}_{6}\right): \delta 8.64(1 \mathrm{H}, \mathrm{s})$, $7.54(1 \mathrm{H}, \mathrm{s}), 7.41-7.28(5 \mathrm{H}, \mathrm{m}), 6.36(1 \mathrm{H}, \mathrm{dd}, J=3.1,1.8 \mathrm{~Hz}), 6.23(1 \mathrm{H}, \mathrm{d}, J=2.7 \mathrm{~Hz}), 5.04(2 \mathrm{H}$, s), $4.80(1 \mathrm{H}, \mathrm{s}), 4.14(1 \mathrm{H}, \mathrm{s}), 1.24(3 \mathrm{H}, \mathrm{d}, J=6.7 \mathrm{~Hz}) \cdot{ }^{13} \mathrm{C}$ NMR $(100 \mathrm{MHz}$, DMSO-d $): \delta 156.9$, 156.3, 141.7, 137.0, 128.4, 127.8, 127.7, 110.1, 105.8, 65.3, 52.1, 17.8. HRMS (ESI) m/z: $[\mathrm{M}+\mathrm{Na}]^{+}$Calculated for $\mathrm{C}_{14} \mathrm{H}_{16} \mathrm{~N}_{2} \mathrm{O}_{3} \mathrm{Na} 283.1059$; found 283.1054.

Chiral SFC analysis of 14 (Chiralpak AD, $35^{\circ} \mathrm{C}, 100$ bar, cosolvent: $0.1 \%$ DEA in $\mathrm{MeOH}, 2$ $\mathrm{mL} / \mathrm{min}, 0$ min to $4 \mathrm{~min}$ ramp $10-50 \%$ cosolvent then hold $50 \%$ cosolvent for $2 \mathrm{~min}$ )

Racemate:

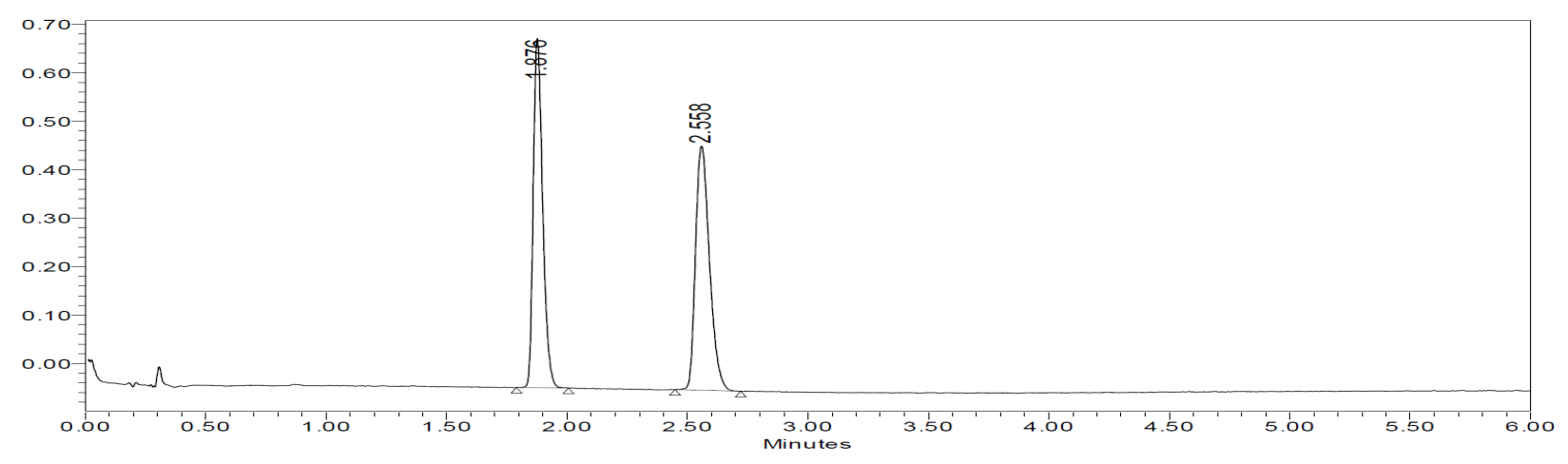

\begin{tabular}{|c|c|c|r|}
\hline & RT & Area & $\%$ Area \\
\hline 1 & 1.876 & 1959992 & 49.11 \\
\hline 2 & 2.558 & 2031072 & 50.89 \\
\hline
\end{tabular}

Reaction Product:

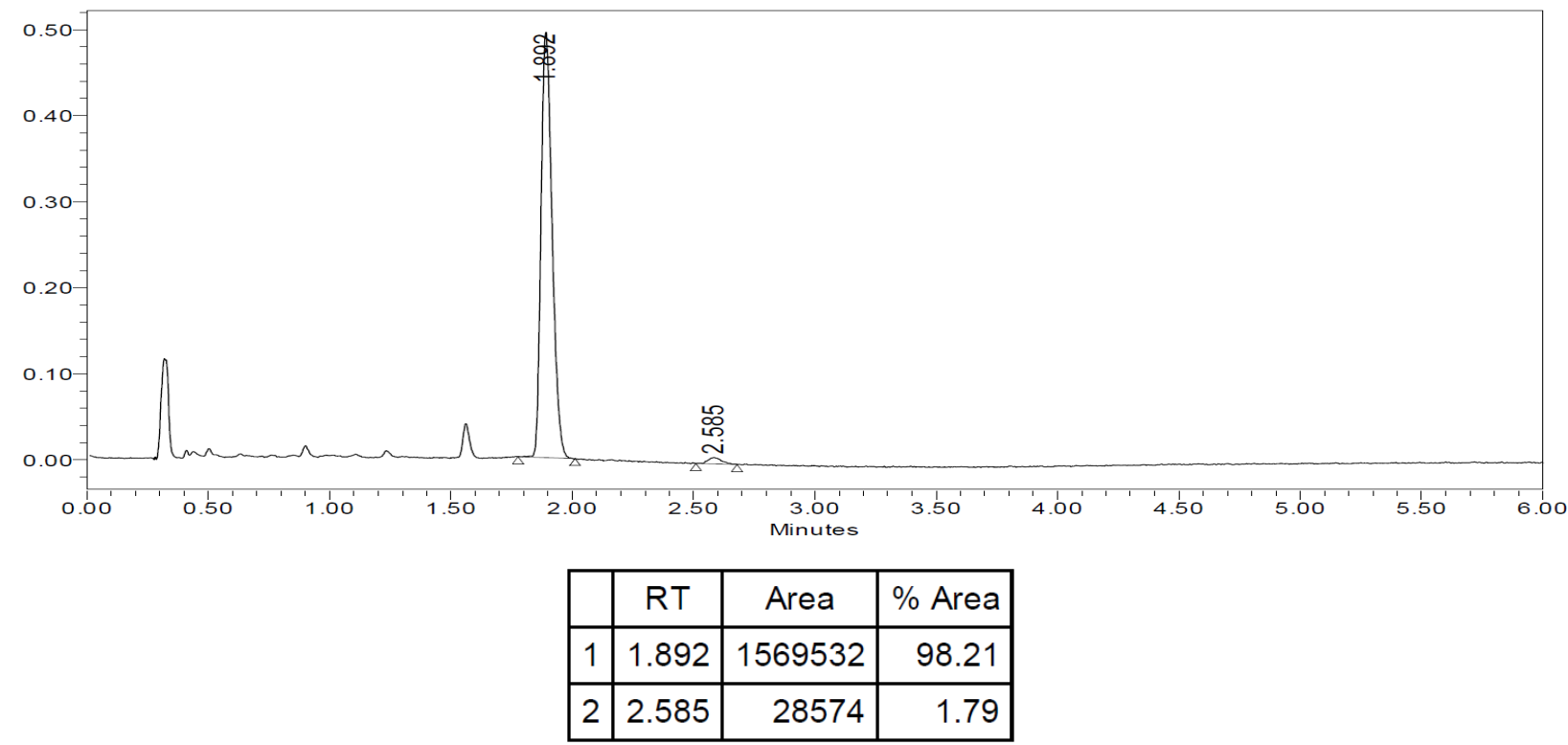




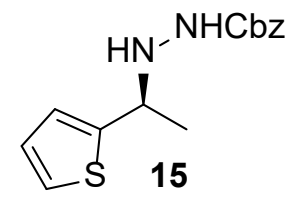

benzyl (S)-2-(1-(thiophen-2-yl)ethyl)hydrazine-1-carboxylate (compound 15, Table 2). Prepared according to the general procedure utilizing benzyl $(E)$ 2-(1-(thiophen-2-yl)ethylidene)hydrazine-1-carboxylate $(274 \mathrm{mg}, 1.00 \mathrm{mmol})$, bis(2-methallyl)(1,5-cyclooctadiene)ruthenium(II) $(3.2 \mathrm{mg}, 1 \mathrm{~mol} \%)$, Walphos ligand SL-W022-1 (7.6 mg, $1.1 \mathrm{~mol} \%), \mathrm{HBF}_{4}-\mathrm{OEt}_{2}(2.7 \mu \mathrm{L}, 2 \mathrm{~mol} \%)$ and hydrogen (100 psi) to give the title product $\left(96 \%, 96 \%\right.$ ee). ${ }^{1} \mathrm{H}$ NMR $\left(400 \mathrm{MHz}, \mathrm{DMSO}-\mathrm{d}_{6}\right): \delta 8.66$ $(1 \mathrm{H}, \mathrm{s}), 7.46-7.23(6 \mathrm{H}, \mathrm{m}), 6.94(1 \mathrm{H}, \mathrm{d}, J=4.1 \mathrm{~Hz}), 5.04(2 \mathrm{H}, \mathrm{s}), 4.91(1 \mathrm{H}, \mathrm{s}), 4.43(1 \mathrm{H}, \mathrm{s}), 1.27$ $(3 \mathrm{H}, \mathrm{d}, J=6.5 \mathrm{~Hz}) .{ }^{13} \mathrm{C}$ NMR $\left(100 \mathrm{MHz}, \mathrm{DMSO}-\mathrm{d}_{6}\right): \delta 157.0,148.0,137.0,128.4,127.8,127.7$, 126.4, 124.4, 124.2, 65.4, 53.9, 22.2. HRMS (ESI) $\mathrm{m} / \mathrm{z}$ : $[\mathrm{M}+\mathrm{Na}]^{+}$Calculated for $\mathrm{C}_{14} \mathrm{H}_{16} \mathrm{~N}_{2} \mathrm{O}_{2} \mathrm{NaS}$ 299.0830; found 299.0827 .

Chiral SFC analysis of 15 (Chiralpak $\mathrm{IH}, 35^{\circ} \mathrm{C}, 100$ bar, cosolvent: $0.1 \%$ DEA in EtOH, $4 \mathrm{~mL} / \mathrm{min}$, isocratic: $10 \%$ cosolvent)

Racemate:

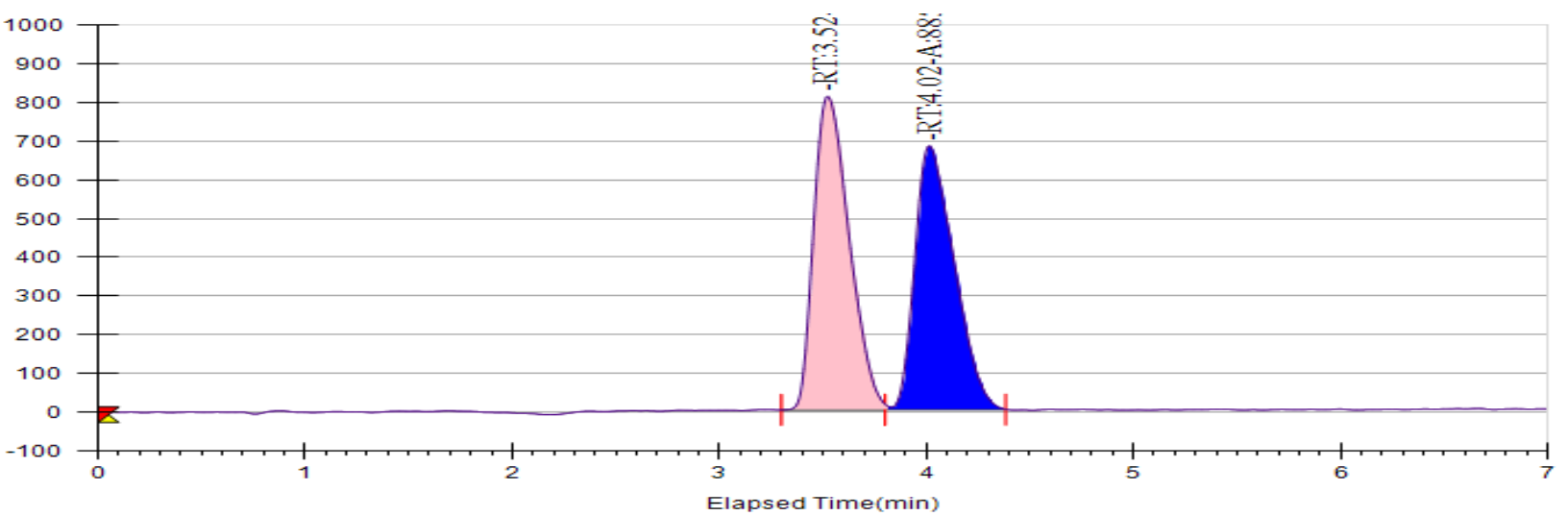

\begin{tabular}{|l|l|l|l|}
\hline Peak No & \% Area & Area & Ret. Time \\
\hline 1 & 51.7058 & 9478.8904 & $3.52 \mathrm{~min}$ \\
\hline 2 & 48.2942 & 8853.4502 & $4.02 \mathrm{~min}$ \\
\hline
\end{tabular}

Reaction Product:

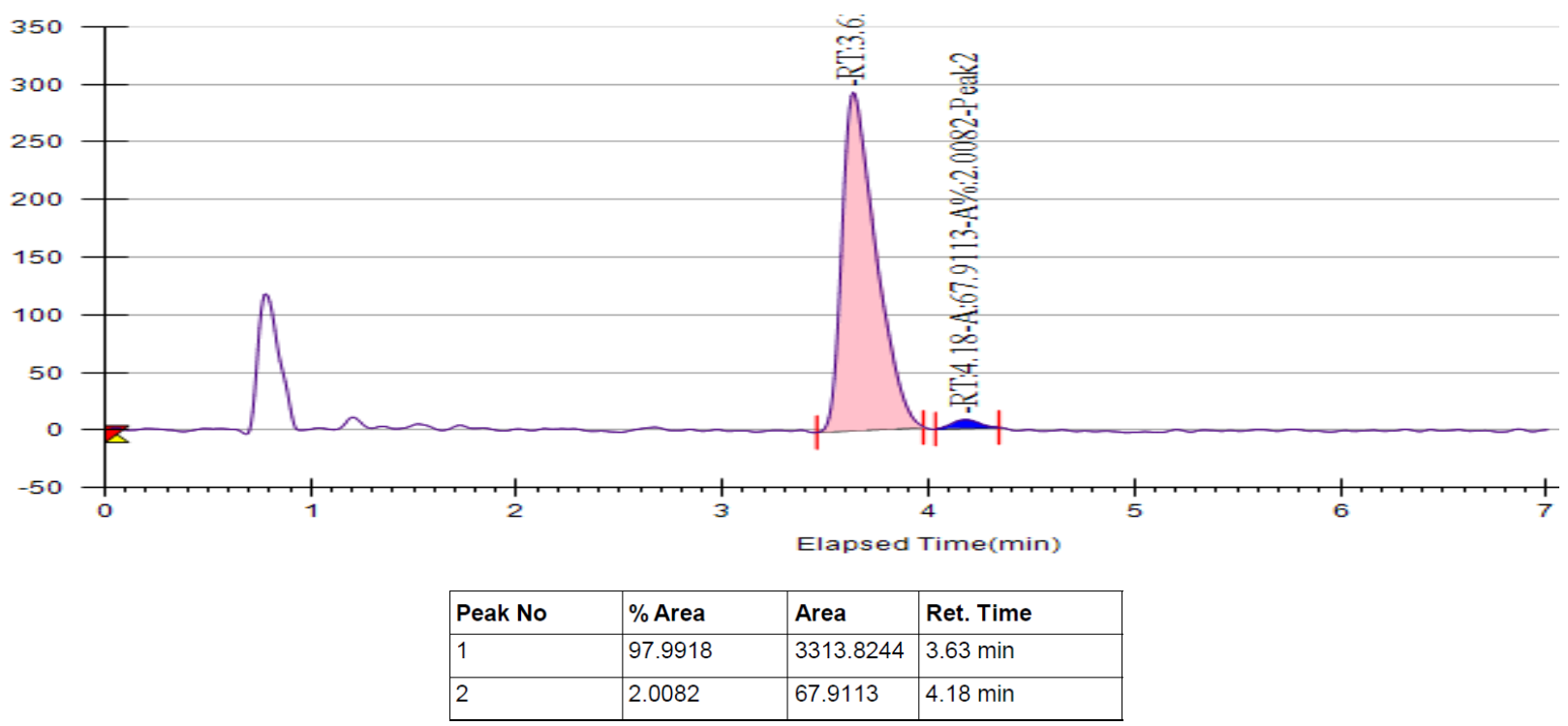


<smiles>CC(=O)NNC(C)c1ccncc1</smiles>

benzyl (S)-2-(1-(pyridin-4-yl)ethyl)hydrazine-1-carboxylate (compound 16, Table 2). Prepared according to the general procedure utilizing benzyl (E)-2-(1-(pyridin-4-yl)ethylidene)hydrazine-1-carboxylate $(269 \mathrm{mg}, \quad 1.00$ mmol), bis(2-methallyl)(1,5-cyclooctadiene)ruthenium(II) $(3.2 \mathrm{mg}, 1 \mathrm{~mol} \%)$, Walphos ligand SL-W022-1 (7.6 mg, 1.1 mol\%), HBF $-\mathrm{HEt}_{2}(2.7 \mu \mathrm{L}, 2 \mathrm{~mol} \%)$ and hydrogen (500 psi) to give the title product $\left(64 \%, 58 \%\right.$ ee). ${ }^{1} \mathrm{H}$ NMR $\left(300 \mathrm{MHz}, \mathrm{DMSO}-\mathrm{d}_{6}\right): \delta$ $8.62(1 \mathrm{H}, \mathrm{s}), 8.47(2 \mathrm{H}, \mathrm{d}, \mathrm{J}=2.6 \mathrm{~Hz}), 7.47-7.16(7 \mathrm{H}, \mathrm{m}), 5.15-4.92(3 \mathrm{H}, \mathrm{m}), 4.16(1 \mathrm{H}, \mathrm{s}), 1.19$ $(3 \mathrm{H}, \mathrm{d}, \mathrm{J}=6.5 \mathrm{~Hz}) .{ }^{13} \mathrm{C}$ NMR $\left(75 \mathrm{MHz}, \mathrm{DMSO}-\mathrm{d}_{6}\right): \delta 156.8,153.2,149.4,137.0,128.3,127.8$, 127.6, 122.3, 65.3, 57.5, 21.2. HRMS (ESI) m/z: $[\mathrm{M}+\mathrm{H}]^{+}$Calculated for $\mathrm{C}_{15} \mathrm{H}_{18} \mathrm{~N}_{3} \mathrm{O}_{2} 272.1399$; found 272.1400 .

Chiral SFC analysis of 16 (Chiralcel OJ, $35^{\circ} \mathrm{C}, 100$ bar, cosolvent: $0.1 \%$ DEA in $\mathrm{MeOH}, 2 \mathrm{~mL} / \mathrm{min}$, isocratic: $10 \%$ cosolvent)

Racemate:

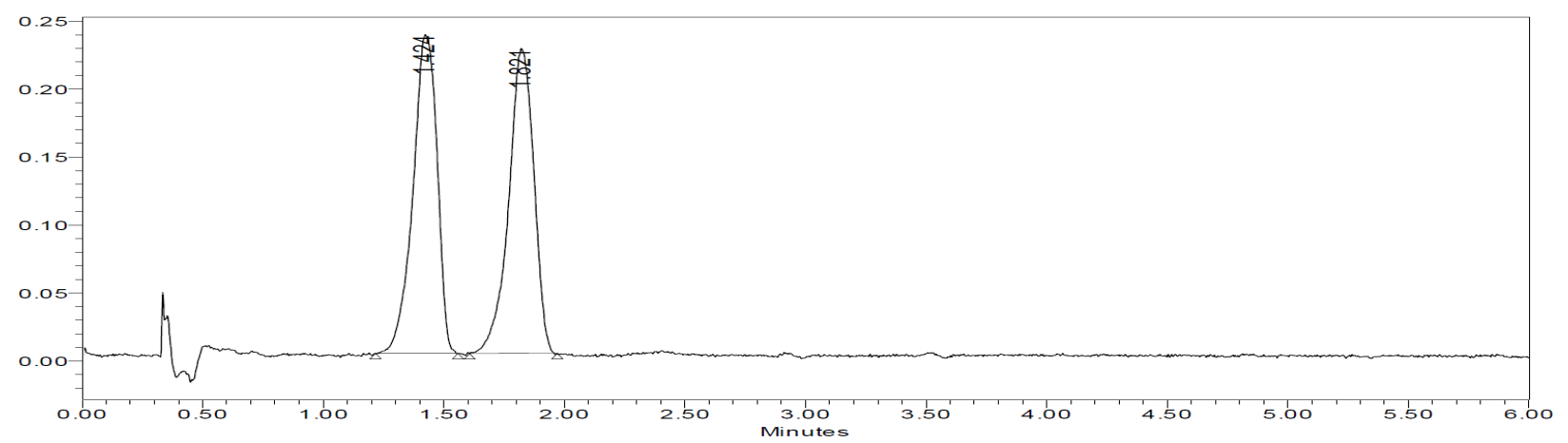

\begin{tabular}{|c|c|c|r|}
\hline & RT & Area & \% Area \\
\hline 1 & 1.424 & 1635294 & 50.05 \\
\hline 2 & 1.821 & 1631815 & 49.95 \\
\hline
\end{tabular}

Reaction Product:

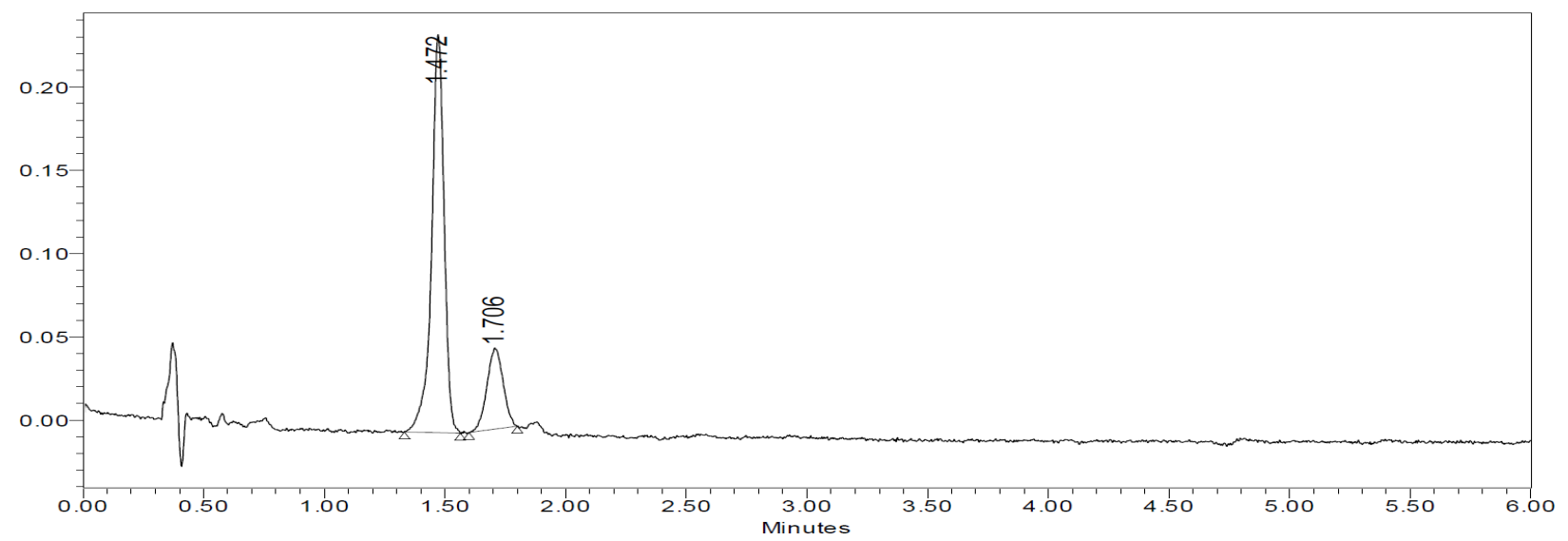

\begin{tabular}{|c|c|c|r|}
\hline & RT & Area & $\%$ Area \\
\hline 1 & 1.472 & 863660 & 79.18 \\
\hline 2 & 1.706 & 227132 & 20.82 \\
\hline
\end{tabular}


$\begin{array}{lll}\mathrm{HN}^{-} \mathrm{NHCbz} & \text { benzyl (S)-2-(1-methoxy-1-oxopropan-2-yl)hydrazine-1-carboxylate } \\ \text { (compound 17, Table 2). Prepared according to the general procedure }\end{array}$ utilizing benzyl (E)-2-(1-methoxy-1-oxopropan-2-ylidene)hydrazine-1carboxylate $(250 \mathrm{mg}, \quad 1.00 \quad \mathrm{mmol}), \quad$ bis(2-methallyl)(1,5cyclooctadiene)ruthenium(II) (3.2 mg, $1 \mathrm{~mol} \%$ ), Walphos ligand SL-W022-1

(7.6 mg, $\left.1.1 \mathrm{~mol}^{2}\right), \mathrm{HBF}_{4}-\mathrm{OEt}_{2}(2.7 \mu \mathrm{L}, 2 \mathrm{~mol} \%)$ and hydrogen (500 psi) to give the title product (87\%, 96\% ee). ${ }^{1} \mathrm{H}$ NMR (300 MHz, DMSO-d $): \delta 8.64(1 \mathrm{H}, \mathrm{s}), 7.46-7.18(5 \mathrm{H}, \mathrm{m}), 5.04(2 \mathrm{H}, \mathrm{s})$, $4.91(1 \mathrm{H}, \mathrm{s}), 3.69-3.54(4 \mathrm{H}, \mathrm{m}), 1.14(3 \mathrm{H}, \mathrm{d}, J=6.9 \mathrm{~Hz}) .{ }^{13} \mathrm{C}$ NMR $\left(75 \mathrm{MHz}, \mathrm{DMSO}_{-} \mathrm{d}_{6}\right): \delta 173.3$, 156.9, 136.9, 128.3, 127.8, 127.7, 65.5, 57.4, 51.5, 15.7. HRMS (ESI) m/z: [M+H] ${ }^{+}$Calculated for $\mathrm{C}_{12} \mathrm{H}_{17} \mathrm{~N}_{2} \mathrm{O}_{4}$ 253.1188; found 253.1187.

Chiral SFC analysis of 17 (Chiralpak AD, $35^{\circ} \mathrm{C}, 100$ bar, cosolvent: $0.1 \%$ DEA in $\mathrm{MeOH}, 2$ $\mathrm{mL} / \mathrm{min}, 0$ min to $2 \mathrm{~min}$ ramp $10-50 \%$ cosolvent then hold $50 \%$ cosolvent for $1 \mathrm{~min}$ )

Racemate:

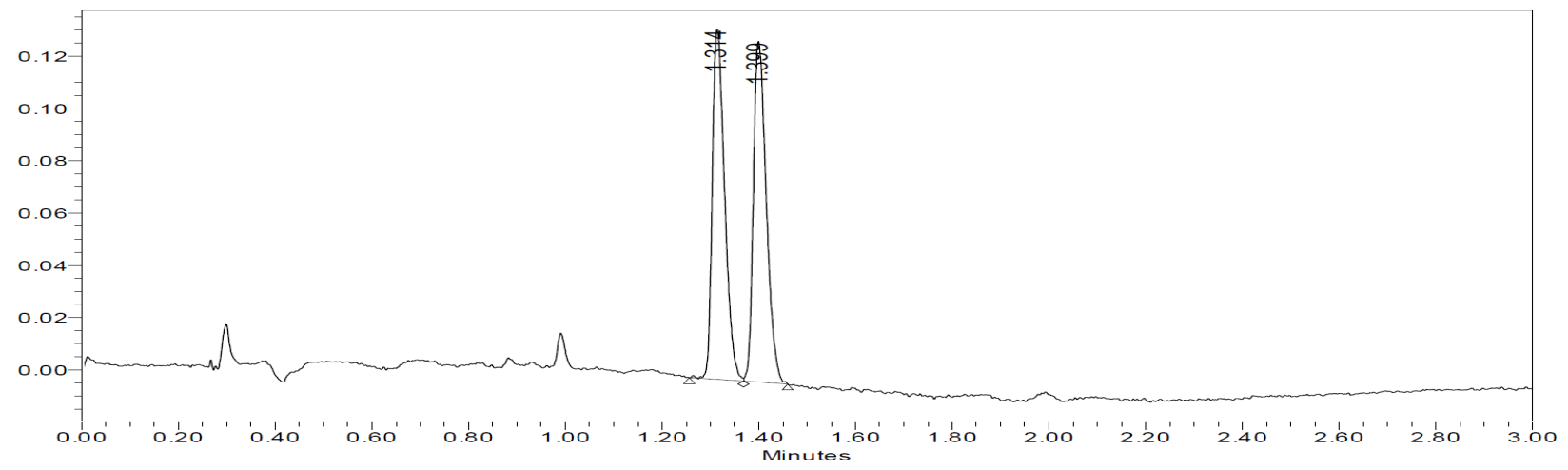

\begin{tabular}{|c|c|c|r|}
\hline & RT & Area & $\%$ Area \\
\hline 1 & 1.314 & 237803 & 49.60 \\
\hline 2 & 1.399 & 241660 & 50.40 \\
\hline
\end{tabular}

Reaction Product:

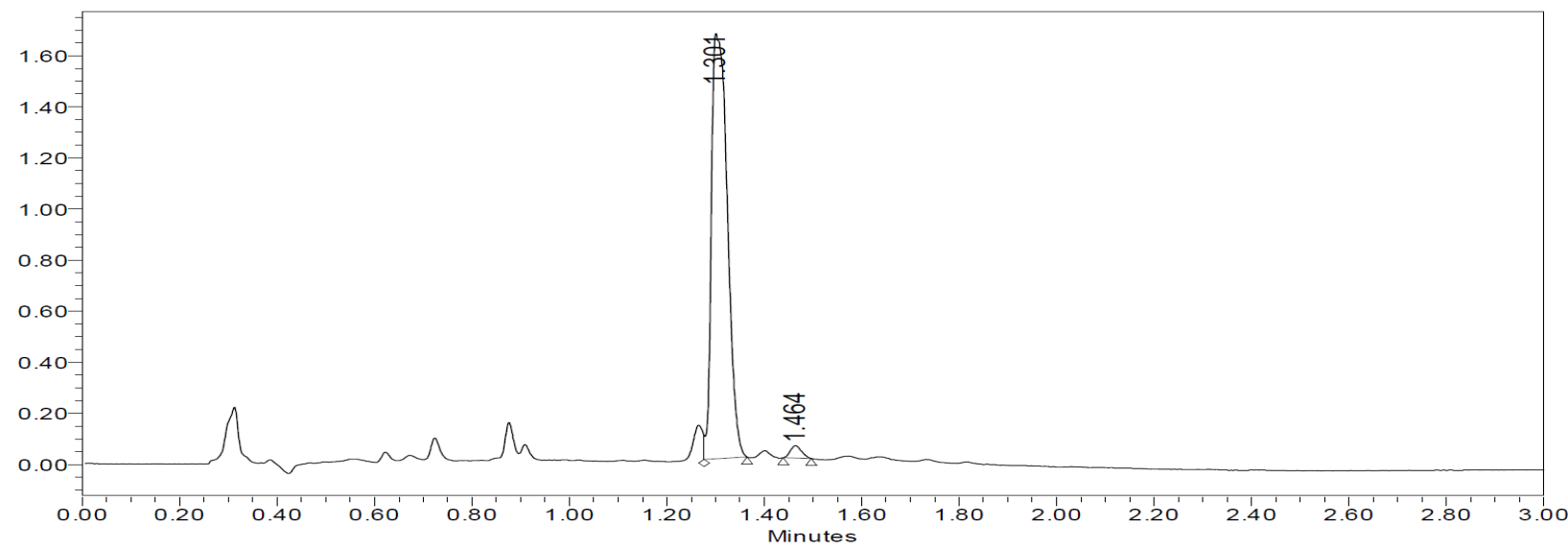

\begin{tabular}{|l|c|c|r|}
\hline & RT & Area & $\%$ Area \\
\hline 1 & 1.301 & 3774400 & 98.00 \\
\hline 2 & 1.464 & 76908 & 2.00 \\
\hline
\end{tabular}




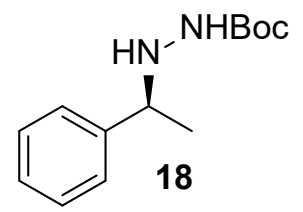

tert-butyl (S)-2-(1-phenylethyl)hydrazine-1-carboxylate (compound 18, Table 3). Prepared according to the general procedure utilizing tert-butyl $(E)$ 2-(1-phenylethylidene)hydrazine-1-carboxylate $(234 \mathrm{mg}, 1.00 \mathrm{mmol})$, bis(2methallyl)(1,5-cyclooctadiene)ruthenium(II) (3.2 $\mathrm{mg}, 1 \mathrm{~mol} \%)$, Walphos ligand SL-W022-1 (7.6 mg, $\left.1.1 \mathrm{~mol}^{\circ}\right), \mathrm{HBF}_{4}-\mathrm{OEt}_{2}(2.7 \mu \mathrm{L}, 2 \mathrm{~mol} \%)$ and hydrogen (100 psi) to give the title product $\left(97 \%, 94 \%\right.$ ee). ${ }^{1} \mathrm{H} \mathrm{NMR}\left(400 \mathrm{MHz}, \mathrm{DMSO}-\mathrm{d}_{6}\right): \delta 8.15(1 \mathrm{H}, \mathrm{s})$, 7.36-7.26 (4H, m), 7.21 (1H, dddd (app tt), J = 6.7, $1.9 \mathrm{~Hz}), 4.56(1 \mathrm{H}, \mathrm{s}), 4.07(1 \mathrm{H}, \mathrm{s}), 1.35(9 \mathrm{H}$, s), $1.17(3 \mathrm{H}, \mathrm{d}, \mathrm{J}=6.6 \mathrm{~Hz}) .{ }^{13} \mathrm{C} \mathrm{NMR}\left(100 \mathrm{MHz}, \mathrm{DMSO}-\mathrm{d}_{6}\right): \delta 156.4,144.3,128.1,127.0,126.8$, 78.2, 58.2, 28.2, 21.7. HRMS (ESI) $\mathrm{m} / \mathrm{z}$ : $[\mathrm{M}+\mathrm{Na}]^{+}$Calculated for $\mathrm{C}_{13} \mathrm{H}_{20} \mathrm{~N}_{2} \mathrm{O}_{2} \mathrm{Na} 259.1422$; found 259.1416 .

Chiral SFC analysis of 18 (Chiralcel AD, $35^{\circ} \mathrm{C}, 100$ bar, cosolvent: $0.1 \%$ DEA in $\mathrm{MeOH}, 2 \mathrm{~mL} / \mathrm{min}$, 0 min to 2 min ramp $5-20 \%$ cosolvent then hold $20 \%$ cosolvent for $1 \mathrm{~min}$ )

Racemate:

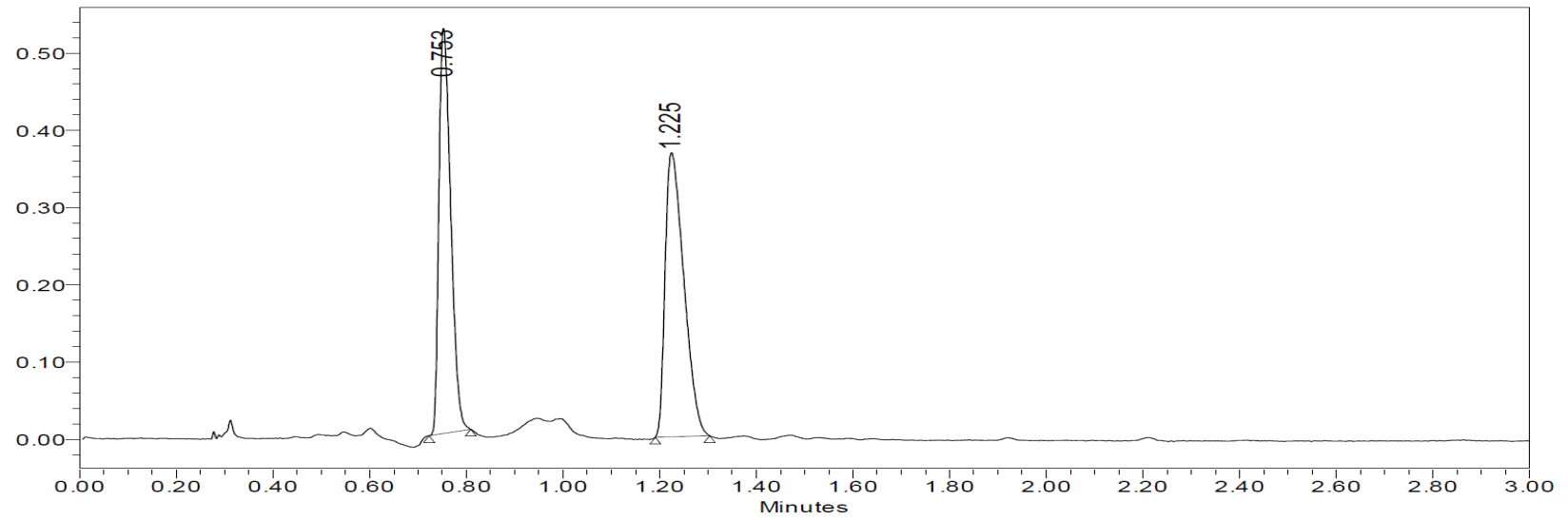

\begin{tabular}{|c|c|c|r|}
\hline & RT & Area & \% Area \\
\hline 1 & 0.753 & 894909 & 47.52 \\
\hline 2 & 1.225 & 988239 & 52.48 \\
\hline
\end{tabular}

Reaction Product:

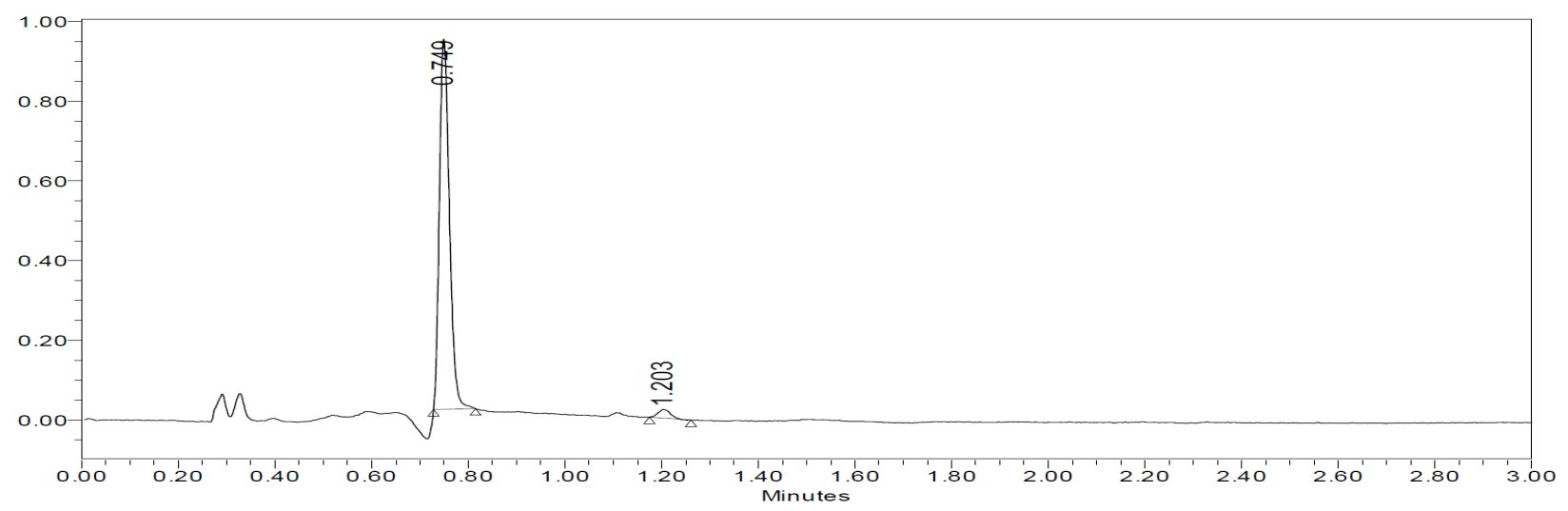

\begin{tabular}{|r|c|r|r|}
\hline & RT & \multicolumn{1}{|c|}{ Area } & \% Area \\
\hline 1 & 0.749 & 1344509 & 97.19 \\
\hline 2 & 1.203 & 38812 & 2.81 \\
\hline
\end{tabular}




\section{Proof of stereochemistry}

Authentic material was prepared as shown below according to the method of Armstrong and coworkers ${ }^{1}$ from commercially sourced $(S)$-1-phenylethylamine.

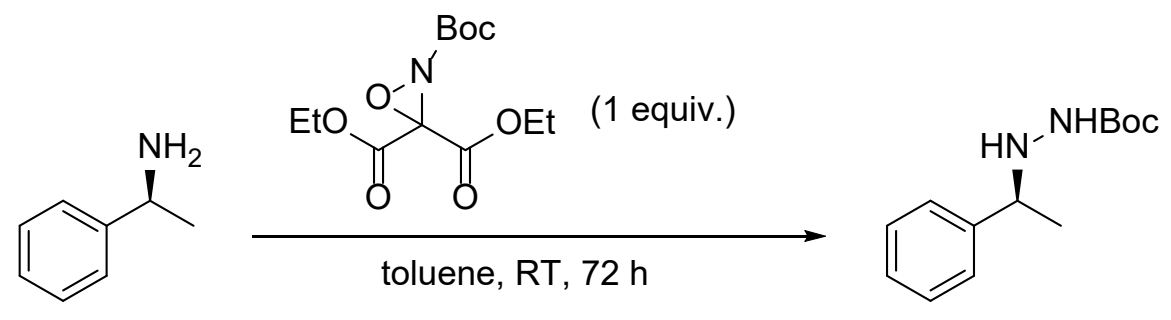

(S)-1-phenylethylamine

(S)-authentic hydrazine

Chiral SFC analysis of authentic 18 (Chiralcel AD (3 micron, $150 \mathrm{~mm}$ ), $35^{\circ} \mathrm{C}, 100 \mathrm{bar}$, cosolvent: $25 \mathrm{mM}$ iBu- $\mathrm{NH}_{2}$ in $\mathrm{MeOH}, 2 \mathrm{~mL} / \mathrm{min}, 0$ min to 5 min ramp 5-20\% cosolvent then hold $20 \%$ cosolvent for $1 \mathrm{~min}$ )

Racemate:

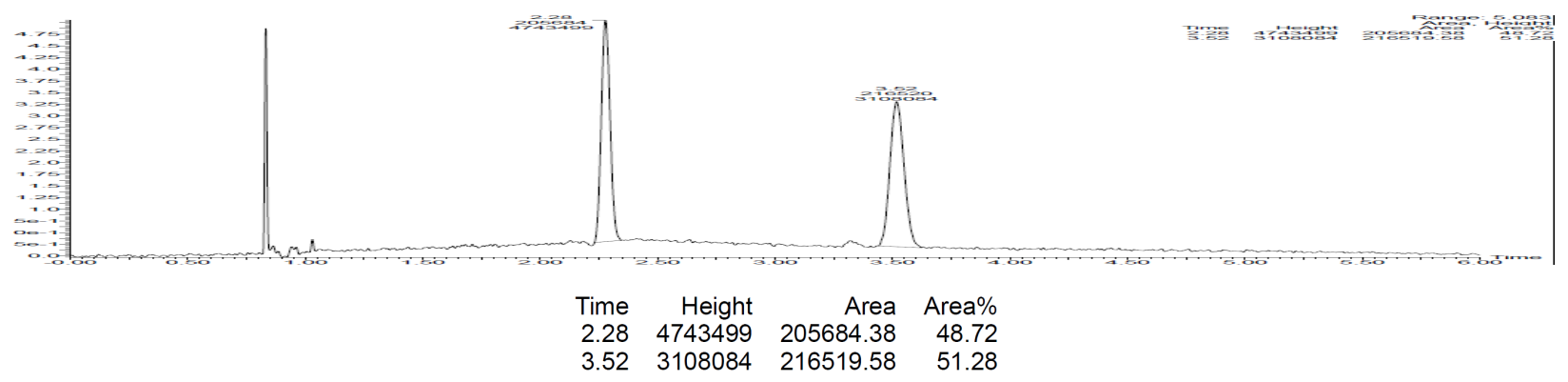

Authentic:

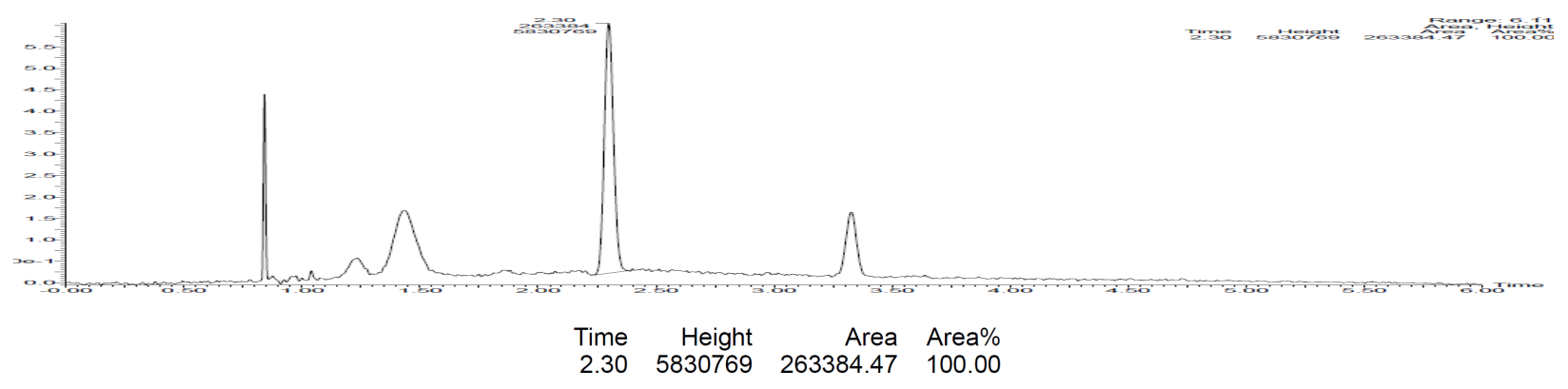

Authentic + Racemate:

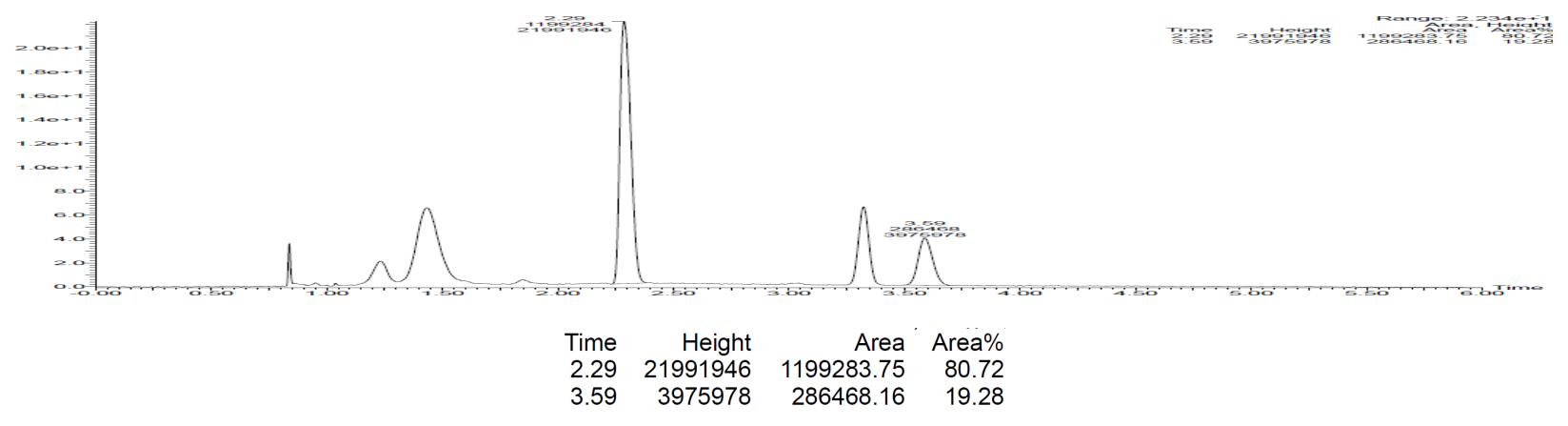




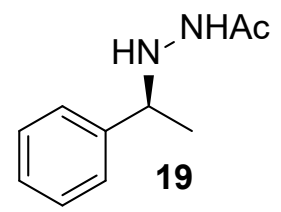

(S)-N'-(1-phenylethyl)acetohydrazide (compound 19, Table 3). Prepared according to the general procedure utilizing (E)-N'-(1phenylethylidene)acetohydrazide $(176 \mathrm{mg}, 1.00 \mathrm{mmol})$, bis(2-methallyl)(1,5cyclooctadiene)ruthenium(II) (3.2 mg, $1 \mathrm{~mol} \%)$, Walphos ligand SL-W022-1 (7.6 $\mathrm{mg}, 1.1 \mathrm{~mol} \%), \mathrm{HBF}_{4}-\mathrm{OEt}_{2}(2.7 \mu \mathrm{L}, 2 \mathrm{~mol} \%$ ) and hydrogen (100 psi) to give the title product $\left(93 \%, 88 \%\right.$ ee). ${ }^{1} \mathrm{H}$ NMR $\left(400 \mathrm{MHz}\right.$, DMSO- $\left.\mathrm{d}_{6}\right)$ :(2 rotamers observed, $\sim 10: 1$ ratio) $\delta$ 9.23 (major, $1 \mathrm{H}, \mathrm{d}, \mathrm{J}=6.1 \mathrm{~Hz}$ ), 8.13 (minor, $1 \mathrm{H}, \mathrm{s}), 7.38-7.28$ (both, $4 \mathrm{H}, \mathrm{m}$ ), 7.28-7.19 (both, $1 \mathrm{H}$, m), 5.18 (minor, $1 \mathrm{H}, \mathrm{d}, \mathrm{J}=2.9 \mathrm{~Hz}$ ), 4.93 (major, $1 \mathrm{H}$, dd, J = 6.2, $2.6 \mathrm{~Hz}$ ), 3.99 (major, 1 H, dddd (app dq), J = 2.5, 6.6 Hz), 3.91 (minor, 1H, dddd (app dq), J = 3.0, $6.6 \mathrm{~Hz}$ ), 1.77 (minor, $3 \mathrm{H}, \mathrm{s}$ ), 1.72 (major, 3H, s), 1.23 (minor, $3 \mathrm{H}, \mathrm{d}, \mathrm{J}=6.6 \mathrm{~Hz}$ ), 1.21 (major, $3 \mathrm{H}, \mathrm{d}, \mathrm{J}=6.6 \mathrm{~Hz}$ ). ${ }^{13} \mathrm{C} \mathrm{NMR}$ (100 MHz, DMSO-d $\mathrm{d}_{6}$ ): (major only) $\delta 173.7,168.5,144.1,128.2,126.9,58.8,21.7,20.6$. HRMS (ESI) $\mathrm{m} / \mathrm{z}:[\mathrm{M}+\mathrm{Na}]^{+}$Calculated for $\mathrm{C}_{10} \mathrm{H}_{14} \mathrm{~N}_{2} \mathrm{ONa} 201.1004$; found 201.1002.

Chiral SFC analysis of 19 (Chiralcel IG, $35^{\circ} \mathrm{C}, 100$ bar, cosolvent: $0.1 \%$ DEA in $\mathrm{MeOH}, 4 \mathrm{~mL} / \mathrm{min}$, 0 min to 2 min ramp $10-50 \%$ cosolvent then hold $50 \%$ cosolvent for $1 \mathrm{~min}$ )

Racemate:

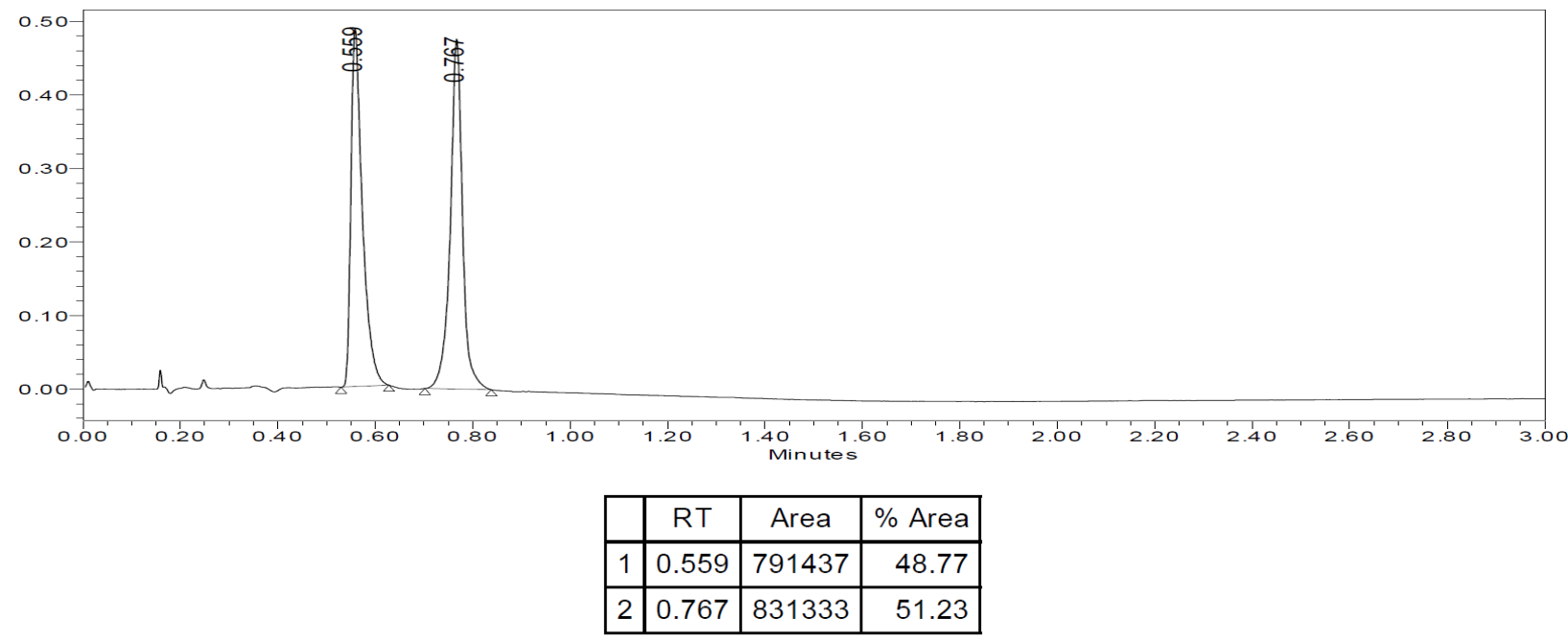

Reaction Product:

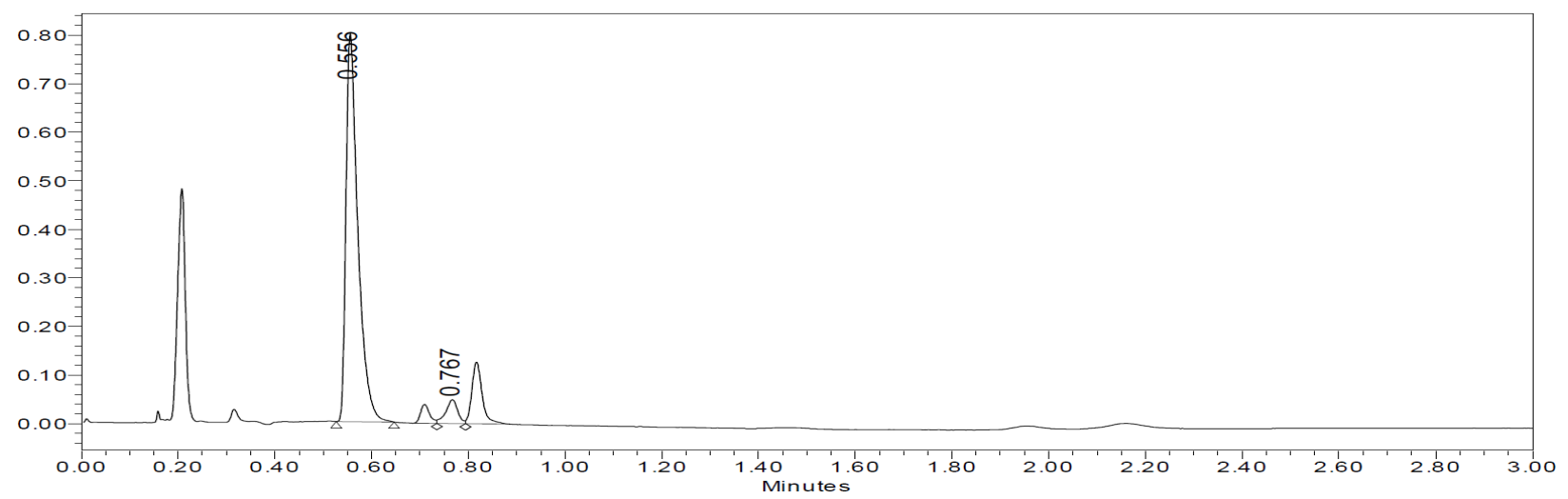

\begin{tabular}{|l|c|r|r|}
\hline & RT & Area & \% Area \\
\hline 1 & 0.556 & 1368020 & 94.20 \\
\hline 2 & 0.767 & 84213 & 5.80 \\
\hline
\end{tabular}




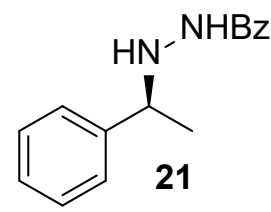

(S)-N'-(1-phenylethyl)benzohydrazide (compound 21, Table 3). Prepared according to the general procedure utilizing (E)-N'-(1phenylethylidene)benzohydrazide $(238 \mathrm{mg}, 1.00 \mathrm{mmol})$, bis(2-methallyl)(1,5cyclooctadiene)ruthenium(II) (3.2 mg, $1 \mathrm{~mol} \%)$, Walphos ligand SL-W022-1 (7.6 $\mathrm{mg}, 1.1 \mathrm{~mol} \%), \mathrm{HBF}_{4}-\mathrm{OEt}_{2}(2.7 \mu \mathrm{L}, 2 \mathrm{~mol} \%)$ and hydrogen (100 psi) to give the title product $\left(96 \%, 52 \%\right.$ ee). ${ }^{1} \mathrm{H}$ NMR $\left(400 \mathrm{MHz}\right.$, DMSO-d $\left.\mathrm{d}_{6}\right): \delta 9.94(1 \mathrm{H}, \mathrm{d}, \mathrm{J}=5.9 \mathrm{~Hz}), 7.75(1 \mathrm{H}$, dd, $\mathrm{J}=7.1,1.4 \mathrm{~Hz}), 7.50(1 \mathrm{H}$, dddd (app tt), J = 7.3, 1.3 Hz), 7.46-7.36 $(4 \mathrm{H}, \mathrm{m}), 7.36-7.28(2 \mathrm{H}$, m), $7.24(1 \mathrm{H}$, dddd (app tt), J = 7.2, 1.3 Hz), $5.26(1 \mathrm{H}$, dd, J = 5.9, $3.5 \mathrm{~Hz}), 4.17(1 \mathrm{H}$, dddd (app $\mathrm{dq}), \mathrm{J}=3.4,6.5 \mathrm{~Hz}), 1.31(3 \mathrm{H}, \mathrm{d}, \mathrm{J}=6.6 \mathrm{~Hz}) .{ }^{13} \mathrm{C} \mathrm{NMR}(100 \mathrm{MHz}$, DMSO-d 6 ): (1 overlapping signal) $\delta 165.9,143.9,133.2,131.2,128.3,128.2,127.1,127.0,58.8,21.5$. HRMS (ESI) m/z: $[\mathrm{M}+\mathrm{Na}]^{+}$Calculated for $\mathrm{C}_{15} \mathrm{H}_{16} \mathrm{~N}_{2} \mathrm{ONa}$ 263.1160; found 263.1158.

Chiral SFC analysis of 21 (Chiralcel IG, $35^{\circ} \mathrm{C}, 100$ bar, cosolvent: $0.1 \%$ DEA in $\mathrm{MeOH}, 4 \mathrm{~mL} / \mathrm{min}$, 0 min to 2 min ramp $10-50 \%$ cosolvent then hold $50 \%$ cosolvent for $1 \mathrm{~min}$ )

Racemate:

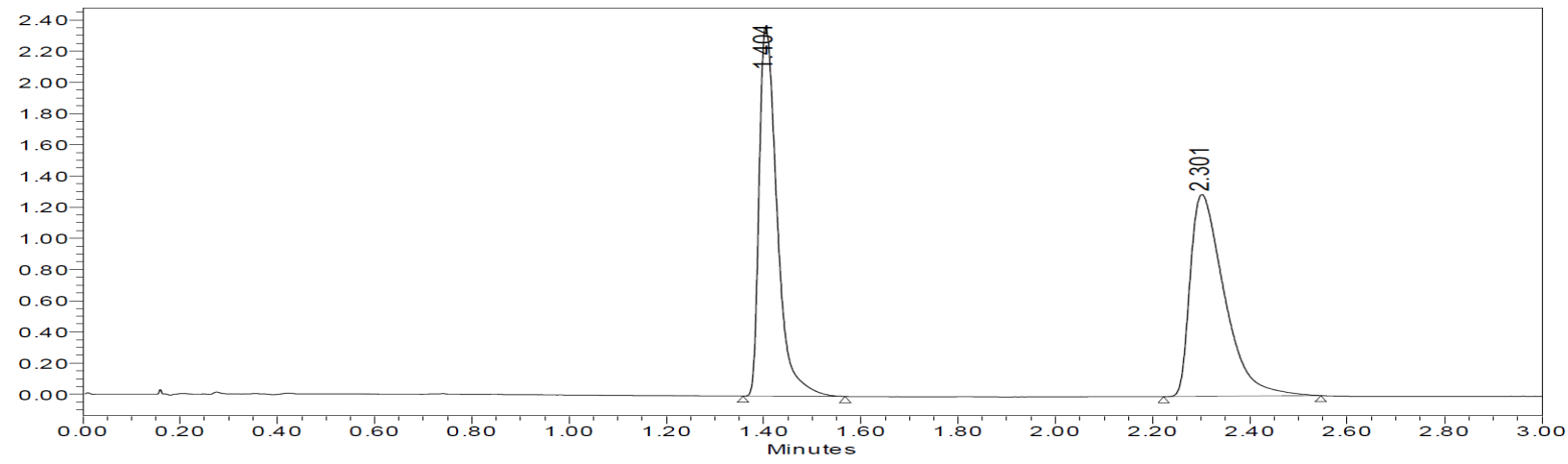

\begin{tabular}{|c|c|c|r|}
\hline & RT & Area & $\%$ Area \\
\hline 1 & 1.404 & 6063285 & 49.03 \\
\hline 2 & 2.301 & 6304385 & 50.97 \\
\hline
\end{tabular}

Reaction Product:

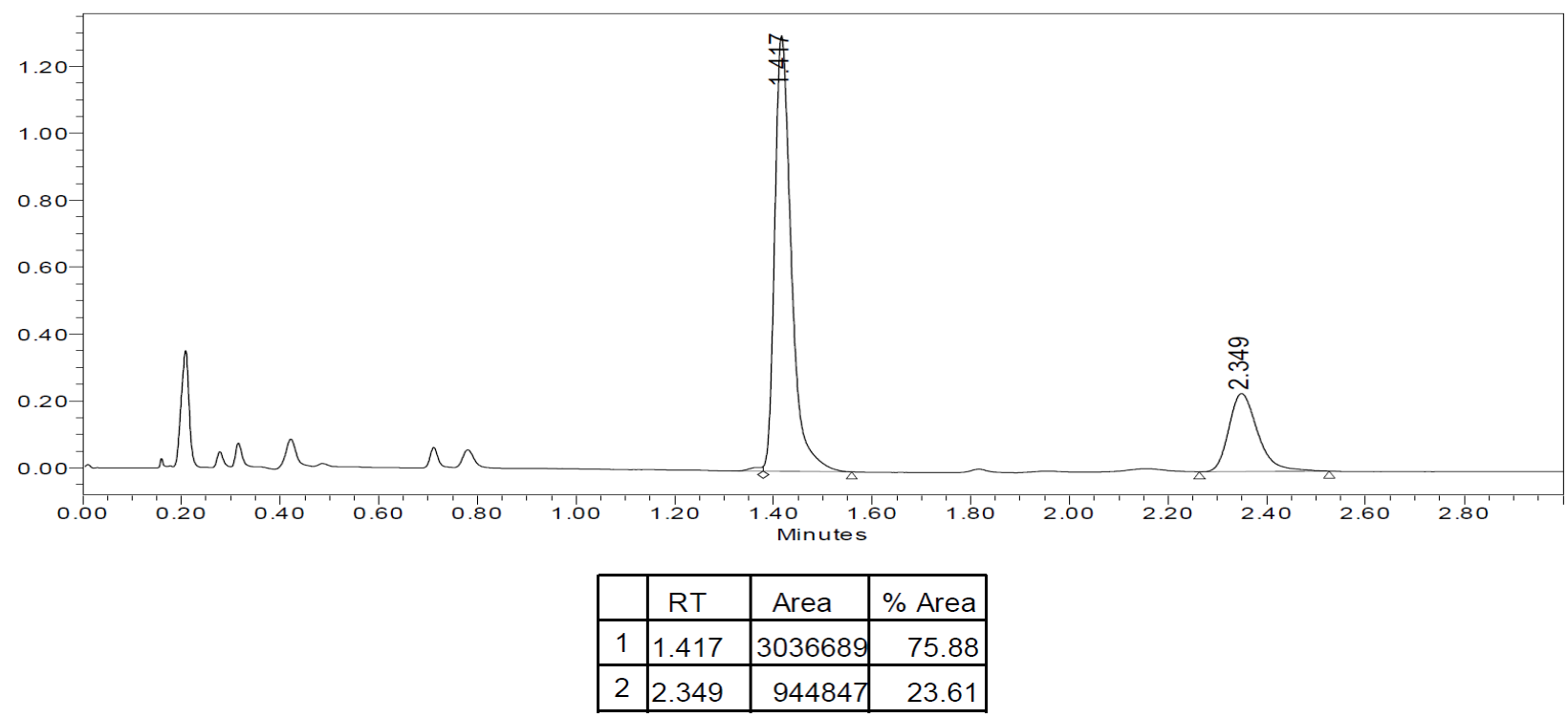


<smiles>COc1ccc(C(=O)NNC(C)c2ccccc2)cc1</smiles>

(S)-4-methoxy-N'-(1-phenylethyl)benzohydrazide (compound 22, Table 3). Prepared according to the general procedure utilizing (E)-4-methoxy-N'-(1-phenylethylidene)benzohydrazide $(268 \mathrm{mg}$, $1.00 \mathrm{mmol})$, bis(2-methallyl)(1,5-cyclooctadiene)ruthenium(II) (3.2 $\mathrm{mg}, 1 \mathrm{~mol} \%)$, Walphos ligand SL-W022-1 (7.6 mg, $1.1 \mathrm{~mol} \%)$, $\mathrm{HBF}_{4}-\mathrm{OEt}_{2}(2.7 \mu \mathrm{L}, 2 \mathrm{~mol} \%)$ and hydrogen (100 psi) to give the title product (88\%, 46\% ee). ${ }^{1} \mathrm{H}$ NMR $\left(400 \mathrm{MHz}, \mathrm{DMSO}-\mathrm{d}_{6}\right): \delta 9.83(1 \mathrm{H}, \mathrm{d}, \mathrm{J}=5.8 \mathrm{~Hz}), 7.75(2 \mathrm{H}, \mathrm{d}, \mathrm{J}$ $=8.8 \mathrm{~Hz}), 7.39(2 \mathrm{H}, \mathrm{d}, \mathrm{J}=7.2 \mathrm{~Hz}), 7.32(2 \mathrm{H}, \mathrm{dd}(\operatorname{app~t}), \mathrm{J}=7.3 \mathrm{~Hz}), 7.24(1 \mathrm{H}, \mathrm{t}, \mathrm{J}=7.2 \mathrm{~Hz}), 6.96$ $(2 \mathrm{H}, \mathrm{d}, \mathrm{J}=8.8 \mathrm{~Hz}), 5.20(1 \mathrm{H}, \mathrm{dd}, \mathrm{J}=5.5,3.2 \mathrm{~Hz}), 4.15(1 \mathrm{H}$, dddd (app dq), J = 3.1, $6.4 \mathrm{~Hz}), 3.78$ $(3 \mathrm{H}, \mathrm{s}), 1.30(3 \mathrm{H}, \mathrm{d}, \mathrm{J}=6.6 \mathrm{~Hz}) .{ }^{13} \mathrm{C}$ NMR $(100 \mathrm{MHz}$, DMSO-d $)$ : (1 overlapping signal) $\delta 165.6$, $161.6,144.0,129.0,128.2,127.0,125.3,113.5,58.9,55.3,21.5$. HRMS (ESI) m/z: $[M+H]^{+}$ Calculated for $\mathrm{C}_{16} \mathrm{H}_{19} \mathrm{~N}_{2} \mathrm{O}_{2} 271.1447$; found 271.1448.

Chiral SFC analysis of 22 (Chiralpak IA, $35{ }^{\circ} \mathrm{C}, 100$ bar, cosolvent: $20 \mathrm{mM} \mathrm{NH}_{3}$ in $\mathrm{MeOH}, 4$ $\mathrm{mL} / \mathrm{min}, 0 \mathrm{~min}$ to $4 \mathrm{~min}$ ramp $10-50 \%$ cosolvent then hold $50 \%$ cosolvent for $1 \mathrm{~min}$ )

Racemate:

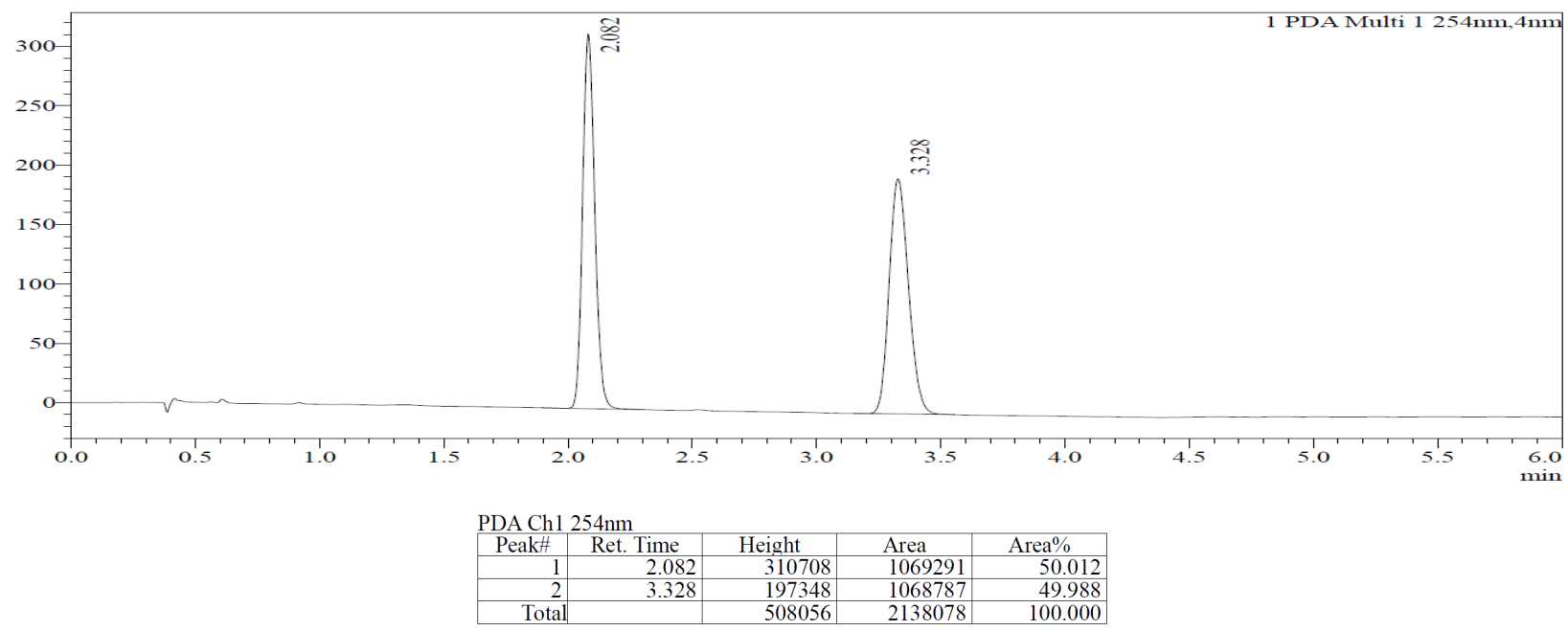

Reaction Product:
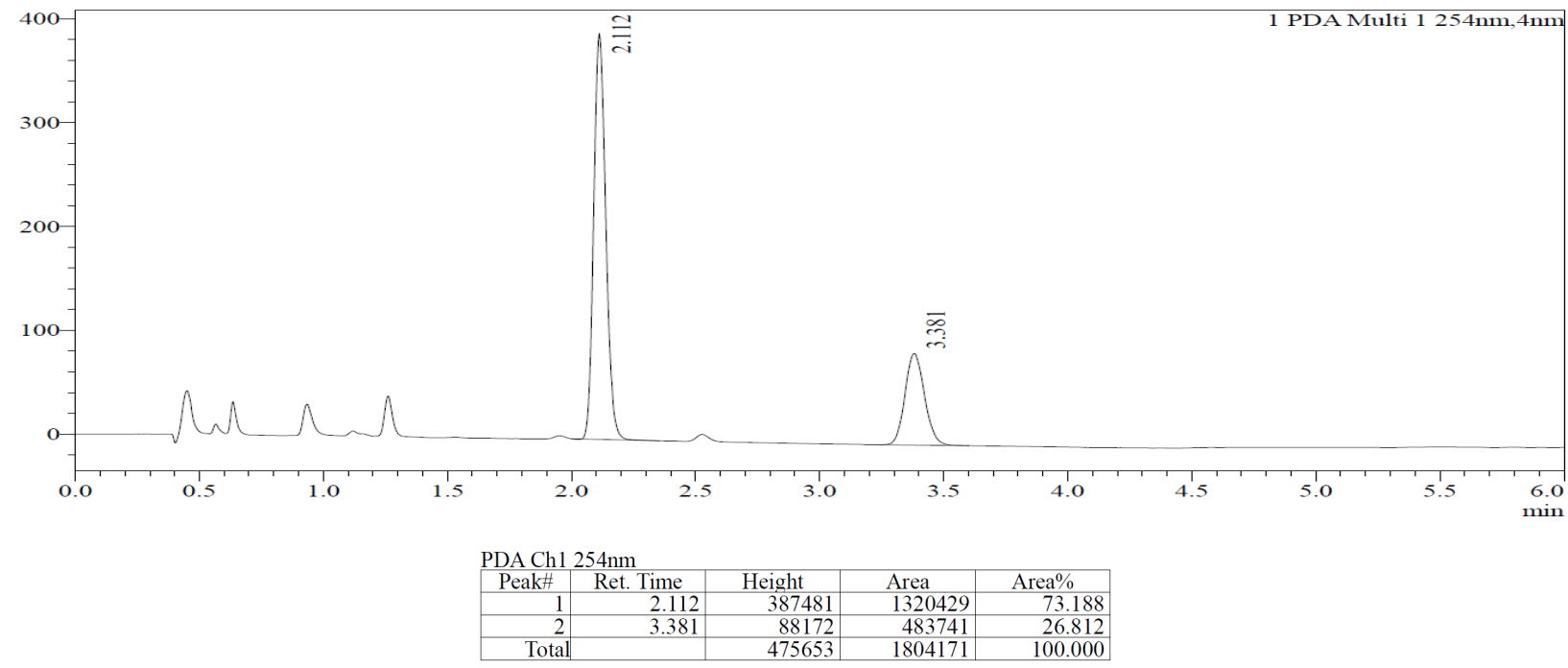
<smiles>C[C@H](NNC(=O)c1ccc(C(F)(F)F)cc1)c1ccccc1</smiles>

(S)-N'-(1-phenylethyl)-4-(trifluoromethyl)benzohydrazide (compound 23, Table 3). Prepared according to the general procedure utilizing (E)-N'-(1-phenylethylidene)-4(trifluoromethyl)benzohydrazide (306 mg, $1.00 \mathrm{mmol}$ ), bis(2methallyl)(1,5-cyclooctadiene)ruthenium(II) (3.2 mg, $1 \mathrm{~mol} \%)$, Walphos ligand SL-W022-1 (7.6 mg, 1.1 mol\%), $\mathrm{HBF}_{4}-\mathrm{OEt}_{2}(2.7 \mu \mathrm{L}$, $2 \mathrm{~mol} \%)$ and hydrogen (100 psi) to give the title product $\left(86 \%, 54 \%\right.$ ee). ${ }^{1} \mathrm{H} \mathrm{NMR}(300 \mathrm{MHz}$, DMSO-d 6 ): $\delta 10.17(1 \mathrm{H}, \mathrm{s}), 7.94(2 \mathrm{H}, \mathrm{d}, \mathrm{J}=8.1 \mathrm{~Hz}), 7.80(2 \mathrm{H}, \mathrm{d}, \mathrm{J}=8.2 \mathrm{~Hz}), 7.40(2 \mathrm{H}, \mathrm{d}, \mathrm{J}=7.3$ $\mathrm{Hz}), 7.32(2 \mathrm{H}, \mathrm{dd}(\mathrm{app} \mathrm{t}), \mathrm{J}=7.1 \mathrm{~Hz}), 7.24(1 \mathrm{H}, \mathrm{t}, \mathrm{J}=7.1 \mathrm{~Hz}), 5.36(1 \mathrm{H}, \mathrm{s}), 4.20(1 \mathrm{H}, \mathrm{q}, \mathrm{J}=6.4$ $\mathrm{Hz}), 1.33(3 \mathrm{H}, \mathrm{d}, \mathrm{J}=6.6 \mathrm{~Hz}) .{ }^{13} \mathrm{C} \mathrm{NMR}\left(75 \mathrm{MHz}, \mathrm{DMSO}-\mathrm{d}_{6}\right): \delta 164.7,143.7,137.0,131.2$ (q, JC$\mathrm{F}=31.9 \mathrm{~Hz}$ ), 128.2, 128.0, 127.0, 127.0, $125.3(\mathrm{q}, \mathrm{JC}-\mathrm{F}=3.8 \mathrm{~Hz}), 123.9$ (q, JC-F = 272.5 Hz), 58.7, 21.4. HRMS (ESI) m/z: $[\mathrm{M}+\mathrm{H}]^{+}$Calculated for $\mathrm{C}_{16} \mathrm{H}_{16} \mathrm{~N}_{2} \mathrm{OF}_{3} 309.1215$; found 309.1212 .

Chiral SFC analysis of 23 (Chiralpak IA, $35^{\circ} \mathrm{C}, 100$ bar, cosolvent: $20 \mathrm{mM} \mathrm{NH}_{3}$ in $\mathrm{MeOH}, 4$ $\mathrm{mL} / \mathrm{min}, 0$ min to $4 \mathrm{~min}$ ramp $10-50 \%$ cosolvent then hold $50 \%$ cosolvent for $1 \mathrm{~min}$ )

Racemate:

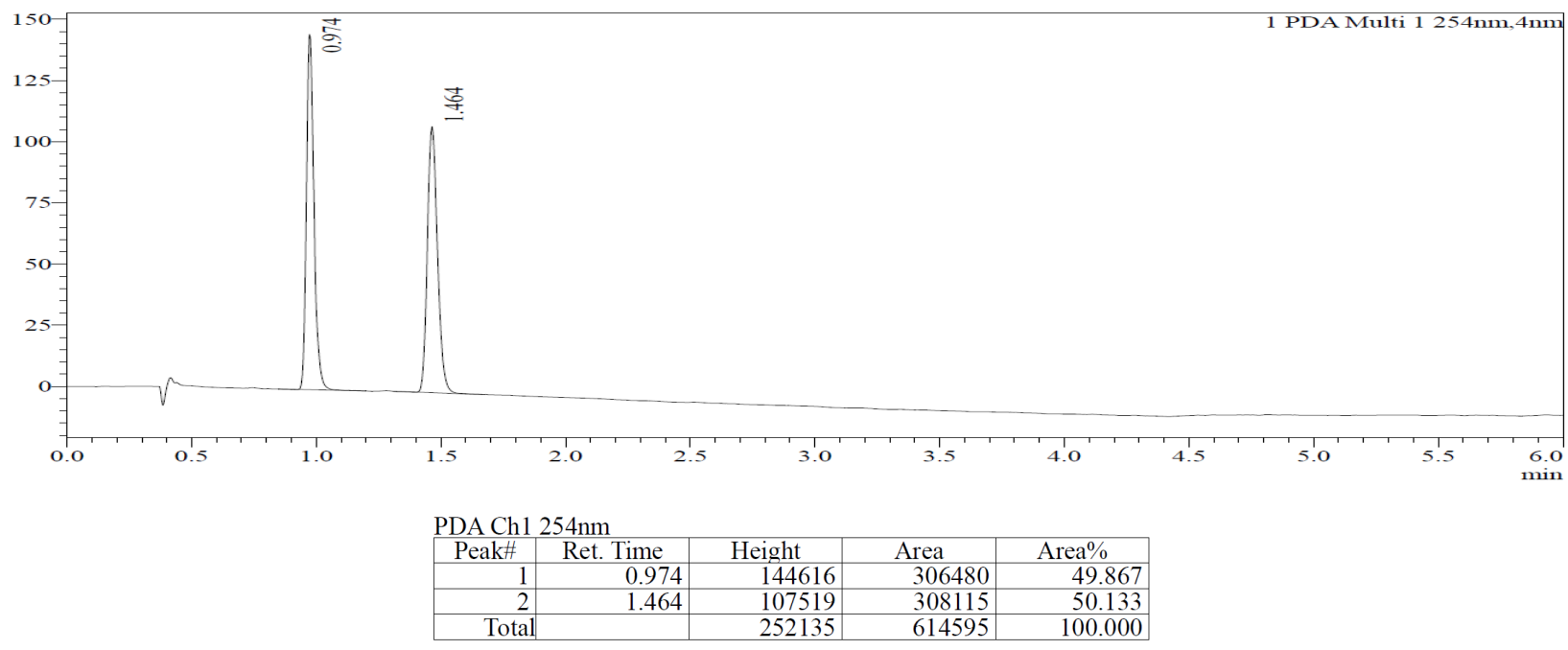

Reaction Product:

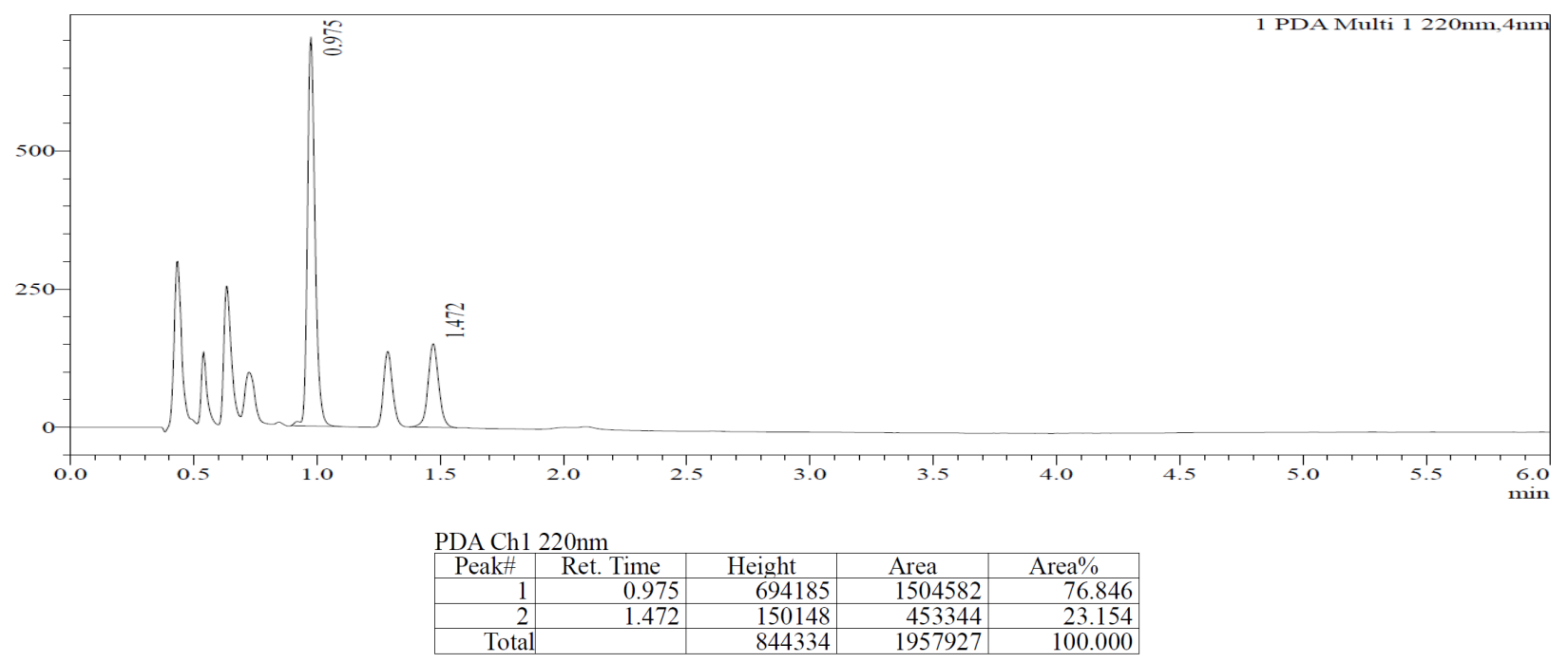




\subsection{Procedure for Large Scale Hydrogenation of 1}

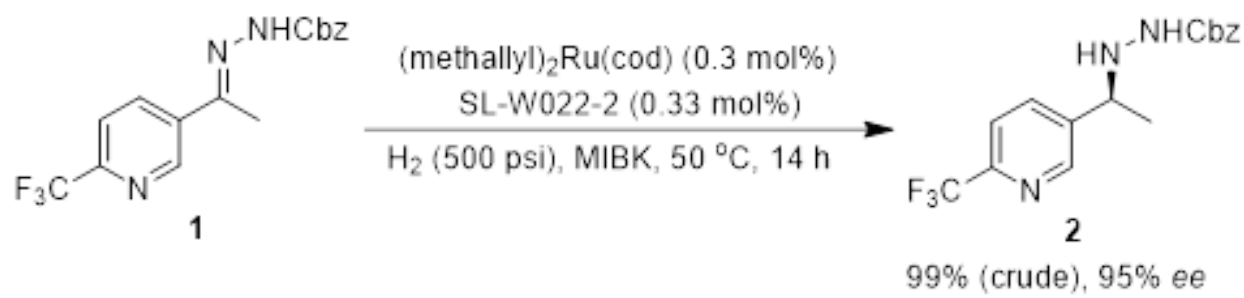

In a nitrogen filled glovebox, bis(2-methallyl)(1,5-cyclooctadiene)ruthenium(II) $(0.48 \mathrm{~g}, 0.3 \mathrm{~mol} \%)$ and Walphos ligand SL-W022-2 (1.16 g, $0.33 \mathrm{~mol} \%)$ were dissolved in dry DCE (35 mL). The resulting solution was stirred for 20 minutes at room temperature followed by addition of $\mathrm{HBF}_{4}$ $\mathrm{OEt}_{2}(0.5 \mathrm{~g}, 0.6 \mathrm{~mol} \%)$. After an additional 20 minutes, the solvent was removed under vacuum. The resulting residue was dissolved in MIBK $(400 \mathrm{~mL})$. In a jacketed pressure vessel, benzyl (E)-2-(1-(6-(trifluoromethyl)pyridin-3-yl)ethylidene)hydrazine-1-carboxylate (170 g, $504 \mathrm{mmol})$ was dissolved in MIBK $(3000 \mathrm{~mL})$ followed by addition of the activated catalyst solution. The vessel was pressurized with $500 \mathrm{psi}_{2}$ and the jacket temperature set to $50^{\circ} \mathrm{C}$ for 14 hours. The reaction was then cooled to room temperature, depressurized and filtered through a pad of silica and concentrated under reduced pressure. Benzyl (S)-2-(1-(6-(trifluoromethyl)pyridin-3yl)ethyl)hydrazine-1-carboxylate (170 g, 99\% yield, 95\% ee) was obtained and used directly in the next step without further purification.

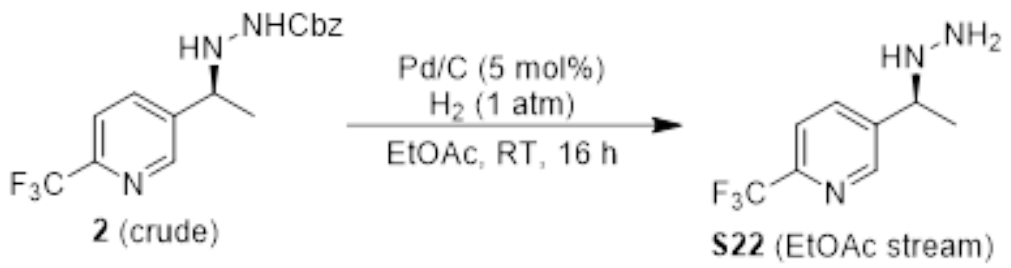

The crude $\mathrm{AH}$ product $(170 \mathrm{~g}, 501 \mathrm{mmol})$ was dissolved in ethyl acetate $(1700 \mathrm{~mL})$ followed by addition of $\mathrm{Pd} / \mathrm{C}(17 \mathrm{~g}, 5 \mathrm{~mol} \%)$. The resulting mixture was stirred under $1 \mathrm{~atm}$ of $\mathrm{H}_{2}$ at room temperature for 16 hours. The $\mathrm{Pd} / \mathrm{C}$ was then filtered off and the filtrate was used in the next step without further purification.

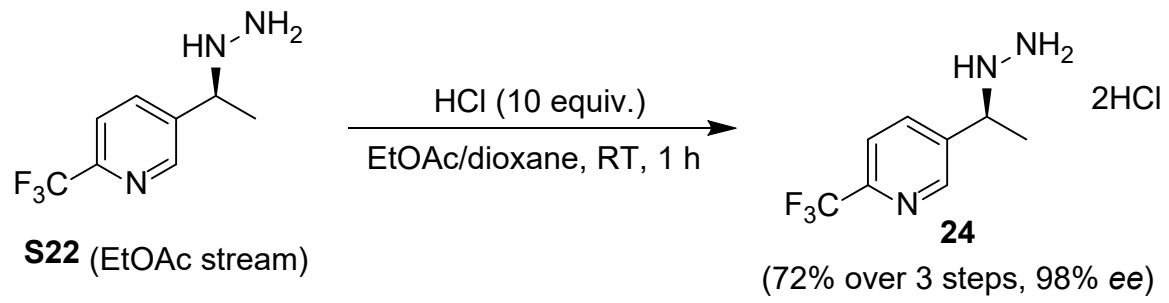

To the crude mono-substituted hydrazine $\mathbf{S 2 2}$ (102 g, in $1700 \mathrm{~mL}$ of ethyl acetate from last step) was added $\mathrm{HCl}$ in dioxane $(4 \mathrm{M}, 1250 \mathrm{~mL}, 10$ equiv.). The resulting solution was stirred at room temperature for 1 hour, resulting in a solid precipitate. The solvent was removed under reduced pressure and the resulting solid was slurried in ethyl acetate $(500 \mathrm{~mL})$ for 4 hours. The slurry was then filtered to obtain the desired product as a white solid (104 g, $359 \mathrm{mmol}, 72 \%$ over 3 steps, $98 \%$ ee). 


\subsection{Additional Screening Data}

\section{Ligand Library Screen}

192 ligands were screened for the hydrogenation of hydrazone 1 according to the general procedure with the following conditions: $10 \mathrm{~mol} \% \mathrm{Ru}, 11 \mathrm{~mol} \%$ ligand, $\mathrm{MeOH}(0.02 \mathrm{M}), \mathrm{H}_{2}(500$ psi), $50{ }^{\circ} \mathrm{C}, 14$ hours. Positive ee values refer to $(S)$ product, negative ee values refer to $(R)$.

Stock solutions of (methallyl) ${ }_{2} \mathrm{Ru}(\mathrm{cod})$ in dry DCE $(0.0032 \mathrm{M})$ and $\mathrm{HBF}_{4}$ in dry DCE (0.0064 M) were prepared in the glove box and $50 \mu \mathrm{L}$ of each solution was added to $1921-\mathrm{mL}$ vials in two 96-well plates containing the pre-plated ligands. After stirring for 20 minutes at room temperature, the solvent was removed under reduced pressure in a Genevac centrifugal evaporator. A stock solution of hydrazone 1 in dry $\mathrm{MeOH}(0.016 \mathrm{M})$ was prepared and $100 \mu \mathrm{L}$ was distributed to each vial. The plates were sealed in an autoclave, pressurized with $\mathrm{H}_{2}(500 \mathrm{psi})$ and heated to $50{ }^{\circ} \mathrm{C}$ for 14 hours. Conversions and enantioselectivities were obtained by chiral SFC analysis of the reaction mixtures.

\begin{tabular}{|c|c|c|c|c|c|}
\hline Ligand & Shorthand & Name & Vendor & $\begin{array}{l}\text { Conversion } \\
(\%)\end{array}$ & $\begin{array}{l}e e \\
(\%)\end{array}$ \\
\hline L1 & $(\mathrm{S}, \mathrm{S})-\mathrm{bpm} 2$ & $\begin{array}{l}\text { (2S,4S)-1-tert-Butoxycarbonyl-4-diphenylphosphino-2- } \\
\text { (diphenylphosphinomethyl)pyrrolidine }\end{array}$ & Strem & 22.4 & 19.8 \\
\hline L2 & catASium D(R) & (3R,4R)-3,4-Bis(diphenylphosphino)-1-benzylpyrrolidine & Evonik & 1.8 & - \\
\hline L3 & SL-J109-1 & $\begin{array}{l}\text { (R)-1-[(SP)-2-(Diphenylphosphino)ferrocenyl]ethylbis(3,5-dimethyl-3- } \\
\text { methoxyphenyl)phosphine }\end{array}$ & Solvias & 5.6 & 29.1 \\
\hline L4 & SL-T021-2 & $\begin{array}{l}\text { (SP)-1-[(S)-a-Hydroxy-2-(diphenylphosphino)benzyl]-2- } \\
\text { diphenylphosphinoferrocene }\end{array}$ & Solvias & 51.2 & -22.2 \\
\hline L5 & SL-M004-1 & $\begin{array}{l}\text { (SP,S'P)-1,1'-Bis[bis(4-methoxy-3,5-dimethylphenyl)phosphino]-2,2'-bis[(R)- } \\
\text { a-(dimethylamino)benzyl]ferrocene }\end{array}$ & Solvias & 67 & -45.5 \\
\hline L6 & SL-W009-1 & $\begin{array}{l}\text { (R)-1-\{(RP)-2-[2-[Di(3,5-xylyl)phosphino]phenyl]ferrocenyl\}ethyldi(3,5- } \\
\text { xylyl)phosphine }\end{array}$ & Solvias & 97.6 & 38.1 \\
\hline L7 & SL-W018-1 & $\begin{array}{l}\text { (R)-1-\{(RP)-2-[2-(Diphenylphosphino)-4- } \\
\text { (trifluoromethyl)phenyl]ferrocenyl\}ethylbis[3,5-bis- } \\
\text { (trifluoromethyl)phenyl]phosphine }\end{array}$ & Solvias & 62.9 & 21.8 \\
\hline L8 & SL-W019-1 & $\begin{array}{l}\text { (R)-1-\{(RP)-2-[2-(Diphenylphosphino)-4- } \\
\text { methylphenyl]ferrocenyl\}ethylbis[3,5-bis-(trifluoromethyl)phenyl]phosphine }\end{array}$ & Solvias & 40.9 & 17 \\
\hline L9 & SL-W020-1 & $\begin{array}{l}\text { (R)-1-\{(RP)-2-[2-(Diphenylphosphino)-4- } \\
\text { methoxyphenyl]ferrocenyl\}ethylbis[3,5-bis-(trifluoromethyl)phenyl]phosphine }\end{array}$ & Solvias & 34.8 & 15.1 \\
\hline L10 & SL-W021-1 & $\begin{array}{l}\text { (R)-1-\{(RP)-2-[2-(Diphenylphosphino)-4,5- } \\
\text { dimethoxyphenyl]ferrocenyl\}ethylbis[3,5-bis- } \\
\text { (trifluoromethyl)phenyl]phosphine }\end{array}$ & Solvias & 1.7 & - \\
\hline L11 & SL-T025-2 & $\begin{array}{l}\text { (SP)-1-[(S)- } \alpha \text {-Hydroxy-2-(di(3,5-xylyl)phosphino)benzyl]-2-di(3,5- } \\
\text { xylyl)phosphinoferrocene }\end{array}$ & Solvias & 16.9 & 4.6 \\
\hline L12 & $(\mathrm{S}, \mathrm{S})$-DIOP & (4S,5S)-4,5-Bis(diphenylphosphino-methyl)-2,2-dimethyl-1,3-dioxolane & Strem & 2.7 & 26.1 \\
\hline L13 & SL-M001-1 & $\begin{array}{l}\text { (SP,S'P)-1,1'-Bis[(R)- } \alpha \text {-(dimethylamino)benzyl]-2,2'- } \\
\text { bis(diphenylphosphino)ferrocene }\end{array}$ & Solvias & 23.4 & -36.1 \\
\hline L14 & SL-M002-1 & $\begin{array}{l}\text { (SP,S'P)-1,1'-Bis(dicyclohexylphosphino)-2,2'-bis[(R)- } \alpha- \\
\text { (dimethylamino)benzyl]ferrocene }\end{array}$ & Solvias & 85.4 & -20.9 \\
\hline L15 & SL-M003-1 & $\begin{array}{l}\text { (SP,S'P)-1,1'-Bis\{bis[3,5-bis(trifluoromethyl)phenyl]phosphino\}-2,2'-bis[(R)- } \\
\alpha \text {-(dimethylamino)benzyl]ferrocene }\end{array}$ & Solvias & 4.6 & 60.6 \\
\hline L16 & (S)-Binapine & $\begin{array}{l}\text { (3S,3'S,4S,4'S,11bS,11'bS)-(+)-4,4'-Di-t-butyl-4,4',5,5'-tetrahydro-3,3'-bi-3H- } \\
\text { dinaphtho[2,1-c:1',2'-e]phosphepin }\end{array}$ & Chiral Quest & 3.8 & 2.3 \\
\hline L17 & SL-T001-1 & $\begin{array}{l}\text { (RP)-1-[(R)- } \alpha \text {-(Dimethylamino)-2-(diphenylphosphino)benzyl]-2- } \\
\text { diphenylphosphinoferrocene }\end{array}$ & Solvias & 53.9 & 44.6 \\
\hline L18 & SL-T002-1 & $\begin{array}{l}\text { (RP)-1-Dicyclohexylphosphino-2-[(R)- } \alpha \text {-(dimethylamino)-2- } \\
\text { (dicyclohexylphosphino)benzyl]ferrocene }\end{array}$ & Solvias & 5.5 & 30.4 \\
\hline L19 & SL-T026-1 & $\begin{array}{l}\text { (SP)-1-[(S)- } \alpha \text {-Hydroxy-2-(bis(3,5-dimethyl-4- } \\
\text { methoxyphenyl)phosphino)benzyl]-2-diphenylphosphinoferrocene }\end{array}$ & Solvias & 18.6 & -9.5 \\
\hline L20 & SL-J203-2 & $\begin{array}{l}\text { (S)-1-\{(RP)-2-[Bis[3,5-di-tert-butyl-4- } \\
\text { methoxyphenyl]phosphino]ferrocenyl\}ethyldi-tert-butylphosphine }\end{array}$ & Solvias & 62.9 & 56.1 \\
\hline L21 & SL-J413-1 & $\begin{array}{l}\text { (R)-1-\{(SP)-2-[Bis[2-methylphenyl]phosphino]ferrocenyl\}ethylbis(2- } \\
\text { methylphenyl)phosphine }\end{array}$ & Solvias & 39.4 & 12.5 \\
\hline L22 & SL-J301-1 & (R)-1-[(SP)-2-(Diethylphosphino)ferrocenyl]ethyldi-tert-butylphosphine & Solvias & 95.9 & -54.8 \\
\hline L23 & (S)-f-Binaphane & 1,1'-Bis\{(S)-4,5-dihydro-3H-binaphtho[1,2-c:2',1'-e]phosphino\}ferrocene & Chiral Quest & 2.4 & 22.7 \\
\hline L24 & SL-T027-2 & $\begin{array}{l}\text { (SP)-1-[(S)- } \alpha \text {-Hydroxy-2-(bis(3,5- } \\
\text { di(trifluoromethyl)phenyl)phosphino)benzyl]-2-di(3,5- } \\
\text { xylyl)phosphinoferrocene }\end{array}$ & Solvias & 8.3 & 26.5 \\
\hline L25 & (R)-CTH-Jafaphos & (R)-(+)-1,1'-Bis(diphenylphosphino)-2,2'-bis(N,N-diisopropylamido)ferrocene & Strem & 1.9 & - \\
\hline L26 & (R,R)-Me-DuPhos & (-)-1,2-Bis[(2R,5R)-2,5-dimethylphospholano]benzene & Strem & 53.8 & 7.6 \\
\hline
\end{tabular}




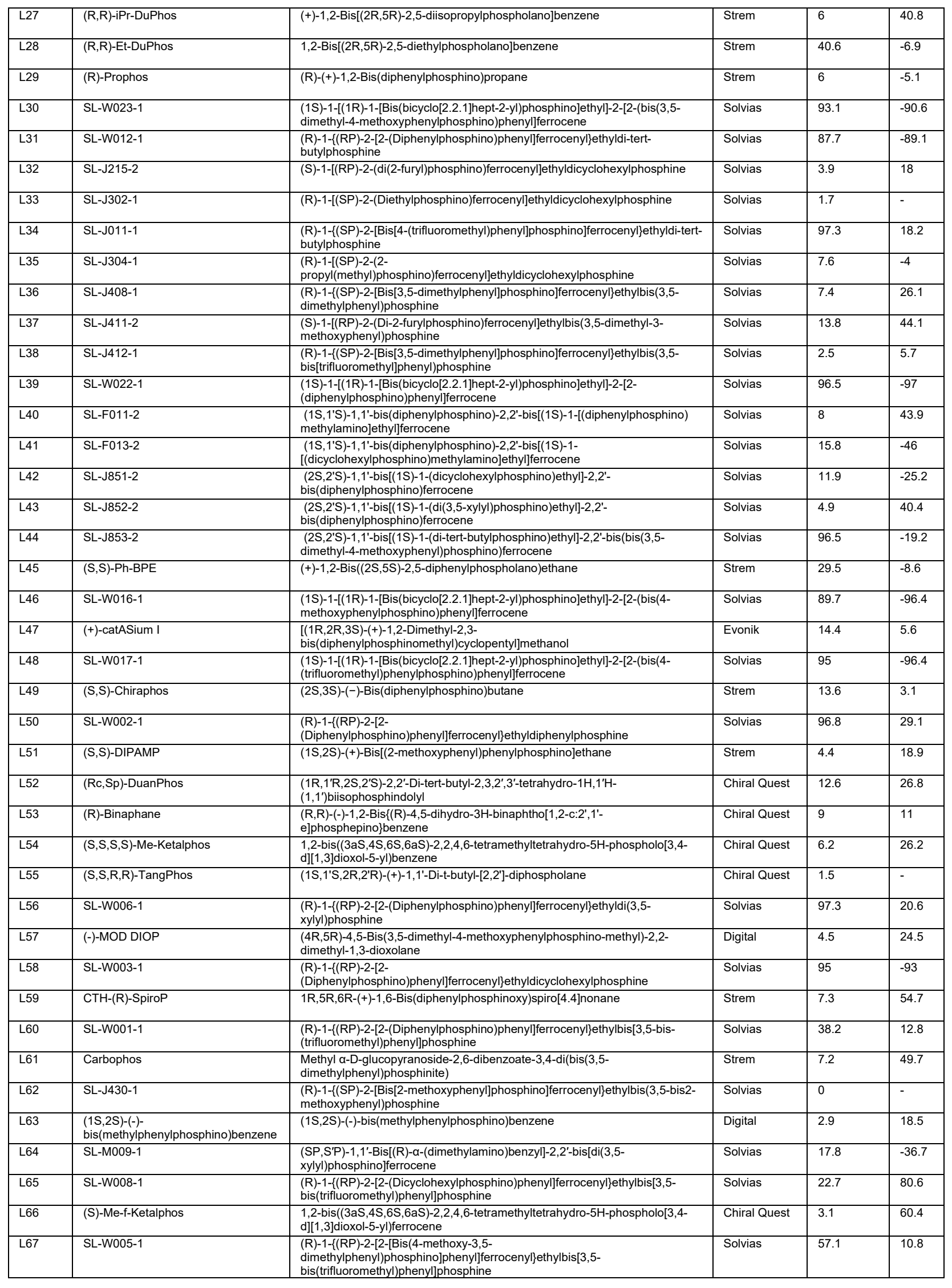




\begin{tabular}{|c|c|c|c|c|c|}
\hline L68 & (R,R)-Norphos & (2R,3R)-(-)-2,3-Bis(diphenylphosphino)-bicyclo[2.2.1]hept-5-ene & Strem & 0.6 & - \\
\hline L69 & catASium $\mathrm{D}(\mathrm{R})$ & (3R,4R)-3,4-Bis(diphenylphosphino)-1-benzylpyrrolidine & Evonik & 92.2 & -48.2 \\
\hline L70 & SL-M012-1 & $\begin{array}{l}\text { (SP,S'P)-1,1'-Bis\{bis[2-methylphenyl]phosphino\}-2,2'-bis[(R)-a- } \\
\text { (dimethylamino)benzyl]ferrocene }\end{array}$ & Solvias & 3.9 & 22 \\
\hline L71 & CTH-(R)-xyl-PhanePhos & (R)-(-)-4,12-Bis[di(3,5-xylyl)phosphino]-[2.2]-paracyclophane & Strem & 5.5 & 77 \\
\hline L72 & $(\mathrm{S}, \mathrm{S})$-BDPP & $(2 \mathrm{~S}, 4 \mathrm{~S})-(-)-2,4$-Bis(diphenylphosphino)pentane & Strem & 2.3 & 21.3 \\
\hline L73 & catASium MNAn(R) & $\begin{array}{l}\text { (-)-2,3-Bis[(2R,5R)-2,5-dimethylphospholano]-N-(4- } \\
\text { methoxyphenyl)maleimide }\end{array}$ & Evonik & 9 & 59.3 \\
\hline$\overline{L 74}$ & catASium MNN(R) & $\begin{array}{l}\text { 4,5-bis((2R,5R)-2,5-dimethylphospholan-1-yl)-1,2-dimethyl-1,2- } \\
\text { dihydropyridazine-3,6-dione }\end{array}$ & Evonik & 5.1 & -2.5 \\
\hline L75 & catASium MNMesF(R) & $\begin{array}{l}\text { 2,3-Bis[(2R,5R)-2,5-dimethylphospholano]-N-[3,5-bis(trifluoromethyl)- } \\
\text { phenyl]maleimide }\end{array}$ & Evonik & 3.8 & 39.2 \\
\hline L76 & catASium MNMes(R) & 2,3-Bis[(2R,5R)-2,5-dimethylphospholano]-N-(3,5-dimethylphenyl)maleimide & Evonik & 0.8 & - \\
\hline L77 & (S,S)-Me-UCAP-DTBM & $\begin{array}{l}\text { (2S,5S)-1-(2-(bis(3,5-di-tert-butyl-4-methoxyphenyl)phosphanyl)phenyl)-2,5- } \\
\text { dimethylphospholane }\end{array}$ & Takasago & 15.1 & -27 \\
\hline L78 & $(\mathrm{R}, \mathrm{R})$-QuinoxP* & $(\mathrm{R}, \mathrm{R})-(-)-2,3-$ Bis(tert-butylmethylphosphino)quinoxaline & Strem & 26.7 & -29.4 \\
\hline L79 & SL-P051-1 & $\begin{array}{l}\text { (2R,5R)-1-(RP)-(2-(diphenylphosphanyl)ferrocenyl)-2,5- } \\
\text { dimethylphospholane }\end{array}$ & Solvias & 2.1 & 9.1 \\
\hline L80 & SL-P053-2 & $\begin{array}{l}\text { (2S,5S)-1-(RP)-(2-(diphenylphosphanyl)ferrocenyl)-2,5- } \\
\text { dimethylphospholane }\end{array}$ & Solvias & 11.5 & -57.3 \\
\hline L81 & SL-J005-1 & (R)-1-[(SP)-2-(Diphenylphosphino)ferrocenyl]ethyldi(3,5-xylyl)phosphine & Solvias & 5.6 & 5.1 \\
\hline$\overline{L 82}$ & SL-J014-1 & $\begin{array}{l}\text { (R)-1-\{(SP)-2-[Bis[4-fluorophenyl]jphosphino]ferrocenyl\}ethyldi-tert- } \\
\text { butylphosphine }\end{array}$ & Solvias & 97 & 16.6 \\
\hline L83 & SL-J031-1 & (R)-1-[(SP)-2-(Diethylphosphino)ferrocenyl]ethyldicyclopentylphosphine & Solvias & 37.7 & 3.9 \\
\hline L84 & SL-J211-1 & $\begin{array}{l}\text { (R)-1-\{(SP)-2-[Bis[2-methylphenyl]phosphino]ferrocenyl\}ethyldi-tert- } \\
\text { butylphosphine }\end{array}$ & Solvias & 85.1 & -73.2 \\
\hline L85 & SL-J213-1 & $\begin{array}{l}\text { (R)-1-\{(SP)-2-[Bis[3,5- } \\
\text { bis[trifluoromethyl]phenyl]phosphino]ferrocenyl\}ethyldicyclopentylphosphine }\end{array}$ & Solvias & 2.4 & 24 \\
\hline L86 & SL-J216-1 & $\begin{array}{l}\text { (R)-1-\{(SP)-2-[Bis[1-naphthyl]phosphino]ferrocenyl\}ethyldi-tert- } \\
\text { butylphosphine }\end{array}$ & Solvias & 68.3 & -35.5 \\
\hline L87 & SL-J219-1 & $\begin{array}{l}\text { (R)-1-\{(SP)-2-[Bis[2-methoxyphenyl]phosphino]ferrocenyl\}ethyldi-tert- } \\
\text { butylphosphine }\end{array}$ & Solvias & 89.7 & -4.9 \\
\hline L88 & SL-J220-1 & $\begin{array}{l}\text { (R)-1-\{(SP)-2-[Bis[3,5-dimethyl-4- } \\
\text { methoxyphenyl]phosphino]ferrocenyl\}ethyldicyclopentylphosphine }\end{array}$ & Solvias & 44.3 & -6.1 \\
\hline L89 & $\begin{array}{l}\text { SL-J221-1 } \\
\text { (1) }\end{array}$ & $\begin{array}{l}\text { (R)-1-\{(SP)-2-[Bis[3,5-dimethyl-4- } \\
\text { methoxyphenyl]phosphino]ferrocenyl\}ethylbis(2,4,4- } \\
\text { trimethylpentyl)phosphine }\end{array}$ & Solvias & 17.8 & 3.7 \\
\hline L90 & SL-J222-1 & $\begin{array}{l}\text { (R)-1-\{(SP)-2-[Bis[3,5-dimethyl-4- } \\
\text { methoxyphenyl]phosphino]ferrocenyl\}ethylbis(2,2-dimethylpropyl)phosphine }\end{array}$ & Solvias & 44.1 & -33.8 \\
\hline L91 & SL-J305-1 & $\begin{array}{l}\text { (R)-1-[(SP)-2-(Dicyclohexylphosphino)ferrocenyl]ethylbis(2- } \\
\text { norbornyl)phosphine }\end{array}$ & Solvias & 98.5 & -65 \\
\hline L92 & SL-J404-1 & $\begin{array}{l}\text { (R)-1-\{(SP)-2-[Bis[3,5-dimethylphenyl]phosphino]ferrocenyl\}ethylbis(1- } \\
\text { naphthyl)phosphine }\end{array}$ & Solvias & 16.1 & 58.7 \\
\hline L93 & SL-J409-1 & (R)-1-\{(SP)-2-diphenylphosphino]ferrocenyl\}ethylbis(1-naphthyl)phosphine & Solvias & 10.6 & 45.5 \\
\hline$\overline{L 94}$ & SL-J502-1 & (R)-1-[(SP)-2-(Di-tert-butylphosphino)ferrocenyl]]ethyldiphenylphosphine & Solvias & 98.2 & -69.1 \\
\hline L95 & SL-J505-1 & $\begin{array}{l}\text { (R)-1-[(SP)-2-(Di-tert-butylphosphino)ferrocenyl]]ethylbis(2- } \\
\text { methylphenyl)phosphine }\end{array}$ & Solvias & 96.2 & -19.2 \\
\hline L96 & SL-J506-1 & $\begin{array}{l}\text { (R)-1-[(SP)-2-(Di-tert-butylphosphino)ferrocenyl]]ethylbis(4- } \\
\text { (trifluoromethyl)phenyl)phosphine }\end{array}$ & Solvias & 98.7 & -67.7 \\
\hline L97 & $(\mathrm{R}, \mathrm{R})-\mathrm{Me}-\mathrm{BPE}$ & (+)-1,2-Bis[(2R,5R)-2,5-dimethylphospholano]ethane & Kanata & 4 & 21.7 \\
\hline L98 & $(R, R)$-Et-BPE & $(+)-1,2-B i s[(2 R, 5 R)-2,5$-diethylphospholano]ethane & Kanata & 3 & 13.9 \\
\hline L99 & $(\mathrm{R}, \mathrm{R})$-iPr-BPE & (-)-1,2-Bis[(2R,5R)-2,5-diisopropylphospholano]ethane & Kanata & 4 & 37.2 \\
\hline L100 & (R,R)-Me-Ferrolane & 1,1'-Bis[(2R,5R)-2,5-dimethylphospholano]ferrocene & Kanata & 18 & -17.7 \\
\hline L101 & $(\mathrm{R}, \mathrm{R})$-Et-Ferrolane & 1,1'-Bis[(2R,5R)-2,5-diethylphospholano]ferrocene & Kanata & 86 & -14.7 \\
\hline L102 & $(\mathrm{R}, \mathrm{R})$-iPr-Ferrolane & 1,1'-Bis[(2R,5R)-2,5-diisopropylphospholano]ferrocene & Kanata & 98 & 85.2 \\
\hline L103 & SL-F131-1 & $\begin{array}{l}\text { 1,1'-Bis[(S)P-[(S)Fc-2-[(R)C-1-(dimethylamino)ethyl] } \\
\text { ferrocenyl]]phenylphosphino]ferrocene }\end{array}$ & Solvias & 2 & 26.5 \\
\hline$\overline{L 104}$ & SL-F356-1 & $\begin{array}{l}\text { 1-Dicyclohexylphosphino-1'-\{(R)-\{(RP)-2-[(S)-1- } \\
\text { (dimethylamino)ethyl]ferrocenyl\}phenylphosphino\}ferrocene }\end{array}$ & Solvias & 24 & -43.1 \\
\hline L105 & SL-J681-1 & $\begin{array}{l}\text { (SP)-1-[(R)-tert-Butylphosphinoyl]-2-[(R)-1- } \\
\text { (diphenylphosphino)ethyl]ferrocene }\end{array}$ & Solvias & 9 & 27.1 \\
\hline L106 & SL-J688-1 & $\begin{array}{l}\text { (SP)-1-[(R)-1-(Di-tert-butylphosphino)ethyl]-2-[(R)- } \\
\text { phenylphosphinoyl]ferrocene }\end{array}$ & Solvias & 12 & 40.5 \\
\hline L107 & (R)-PhanePhos & (R)-(-)-4,12-Bis(diphenylphosphino)-[2.2]-paracyclophane & Strem & 96 & -48.2 \\
\hline L108 & (R)-An-PhanePhos & (R)-4,12-Bis[di(4-methoxyphenyl)phosphino][2.2]paracyclophane & Strem & 95 & -41.1 \\
\hline
\end{tabular}




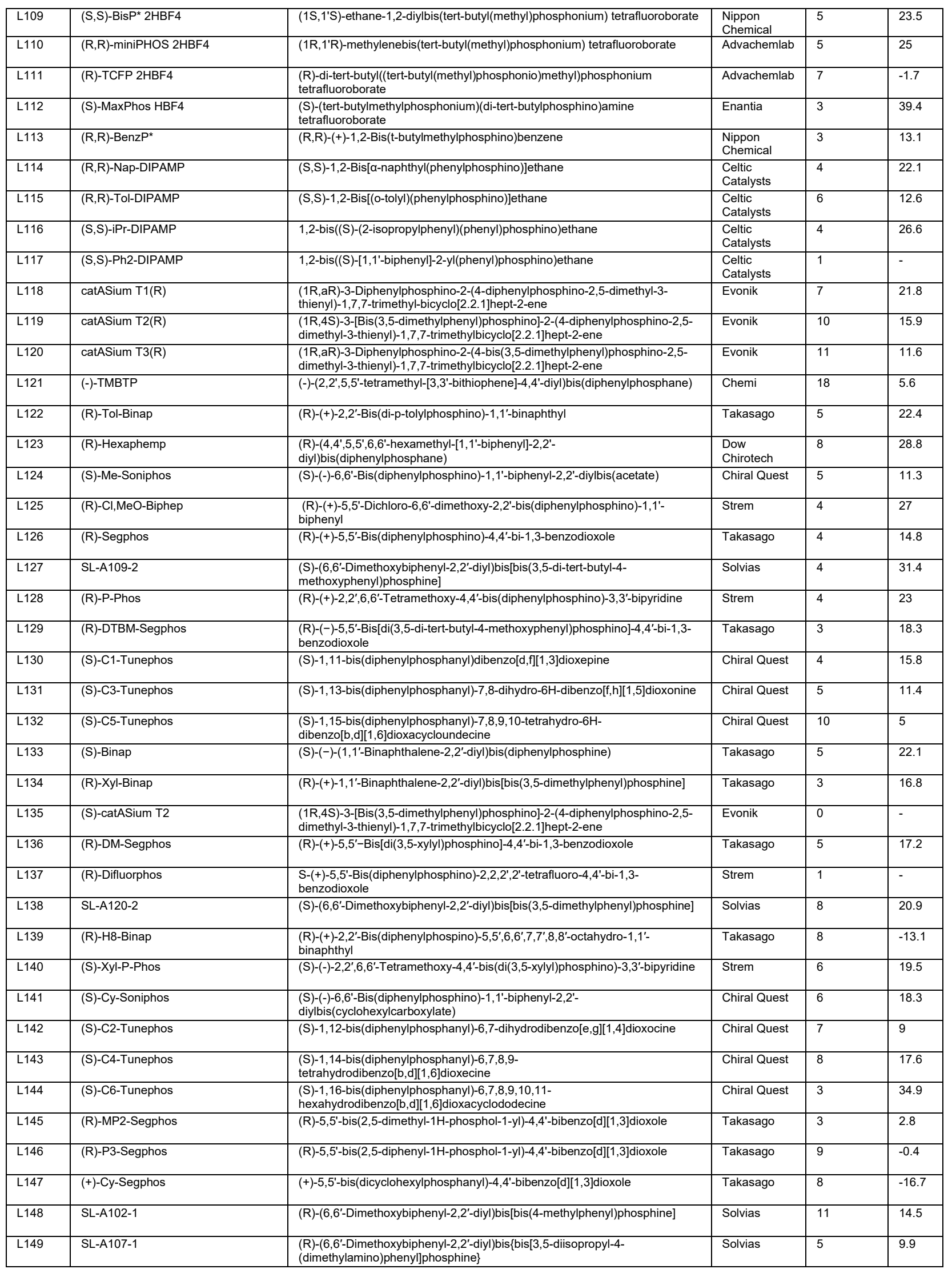




\begin{tabular}{|c|c|c|c|c|c|}
\hline L150 & SL-A104-1 & $\begin{array}{l}\text { (R)-(6,6'-Dimethoxybiphenyl-2,2'-diyl)bis[bis(3,4,5- } \\
\text { trimethoxyphenyl)phosphine] }\end{array}$ & Solvias & 27 & 0.3 \\
\hline L151 & SL-A121-1 & $\begin{array}{l}\text { (R)-(6,6'-Dimethoxybiphenyl-2,2'-diyl)bis[bis(3,5-di-tert- } \\
\text { butylphenyl)phosphine] }\end{array}$ & Solvias & 3 & 19.8 \\
\hline L152 & SL-A108-1 & (R)-(6,6'-Dimethoxybiphenyl-2,2'-diyl)bis(di-2-furylphosphine) & Solvias & 2 & 30.4 \\
\hline L153 & SL-A116-1 & (R)-2,2'-Bis(diisopropylphosphino)-6,6'-dimethoxy-1,1'-biphenyl & Solvias & 16 & 56.6 \\
\hline L154 & SL-A118-1 & (R)-2,2'-Bis(dicyclobutylphosphino)-6,6'-dimethoxy-1,1'-biphenyl & Solvias & 12 & -25.5 \\
\hline L156 & (S)-Synphos & $\begin{array}{l}\text { (S)-(-)-6,6'-Bis(diphenylphosphino)-2,2',3,3'-tetrahydro-5,5'-bi-1,4- } \\
\text { benzodioxin }\end{array}$ & Strem & 15 & 9.7 \\
\hline L157 & (S)-Ph-Garphos & (S)-(4,4',6,6'-Tetramethoxybiphenyl-2,2'-diyl)bis(diphenylphosphine) & Kanata & 8 & 19.2 \\
\hline L158 & (S)-Tol-Garphos & (S)-(4,4',6,6'-Tetramethoxybiphenyl-2,2'-diyl) bis(di-p-tolylphosphine) & Kanata & 11 & 23.4 \\
\hline L159 & (S)-Xyl-Garphos & $\begin{array}{l}\text { (S)-(4,4',6,6'-Tetramethoxybiphenyl -2,2'-diyl)bis(bis(3,5-dimethylphenyl } \\
\text { )phosphine) }\end{array}$ & Kanata & 8 & 13.3 \\
\hline L161 & (S)-DMM-Garphos & $\begin{array}{l}\text { (S)-(4,4',6,6'-Tetramethoxybiphenyl-2,2'-diyl) bis[bis(4-methoxy-3,5- } \\
\text { dimethylphenyl)phosphine] }\end{array}$ & Kanata & 8 & 29 \\
\hline L162 & (S)-DTBM-Garphos & $\begin{array}{l}\text { (S)-(4,4',6,6'-Tetramethoxybiphenyl-2,2'-diyl)bis(bis(3,5-di-tert-butyl-4- } \\
\text { methoxyphenyl)phosphine }\end{array}$ & Kanata & 3 & 13.9 \\
\hline L163 & (R)-DMM-Segphos & $\begin{array}{l}\text { (R)-(+)-5,5'-Bis[di(3,5-dimethyl-4-methoxyphenyl)phosphino]-4,4'-bi-1,3- } \\
\text { benzodioxole }\end{array}$ & Takasago & 8 & 17.8 \\
\hline L164 & (S)-H8-Binam-P & $\begin{array}{l}\text { (S)-(-)-2,2'-Bis(N-diphenylphosphinoamino)-5,5',6,6', 7,7',8, ',-octahydro-1,1'- } \\
\text { binaphthyl }\end{array}$ & Strem & 3 & 23.1 \\
\hline L165 & (S)-Binam-P & (S)-2,2'-Bis(diphenylphosphinoamino)-1,1'-binaphthyl & Strem & 2 & 31.9 \\
\hline L166 & (S)-Xyl-SDP & (S)-(-)-7,7'-Bis[di(3,5-dimethylphenyl)phosphino]-1,1'-spirobiindane & Strem & 22 & -38.4 \\
\hline L167 & (S)-Tol-SDP & $\begin{array}{l}\text { (S)-(-)-7,7'-Bis[di(4-methylphenyl)phosphino]-2,2',3,3'-tetrahydro-1,1'- } \\
\text { spirobiindene }\end{array}$ & Strem & 15 & -22.2 \\
\hline L168 & (S)-SDP & (S)-(-)-7,7'-Bis(diphenylphosphino)-2,2',3,3'-tetrahydro-1,1'-spirobiindene & Strem & 33 & -56.5 \\
\hline L169 & SL-J001-1 & (R)-1-[(SP)-2-(Diphenylphosphino)ferrocenyl]ethyldicyclohexylphosphine & Solvias & 34 & 46.1 \\
\hline L170 & SL-J002-1 & (R)-1-[(SP)-2-(Diphenylphosphino)ferrocenyl]ethyldi-tert-butylphosphine & Solvias & 97 & 13.7 \\
\hline L172 & SL-J006-1 & $\begin{array}{l}\text { (R)-1-\{(SP)-2-[Bis[3,5- } \\
\text { bis(trifluoromethyl)phenyl]phosphino]ferrocenyl\}ethyldicyclohexylphosphine }\end{array}$ & Solvias & 2 & 23.7 \\
\hline L173 & SL-J008-1 & $\begin{array}{l}\text { (R)-1-\{(SP)-2-[Bis[3,5- } \\
\text { bis(trifluoromethyl)phenyl]phosphino]ferrocenyl\}ethyldi(3,5-xylyl)phosphine }\end{array}$ & Solvias & 3 & 21.2 \\
\hline L174 & SL-J204-1 & $\begin{array}{l}\text { (R)-1-\{(SP)-2-[Bis[4- } \\
\text { (trifluoromethyl)phenyl]phosphino]ferrocenyl\}ethyldicyclohexylphosphine }\end{array}$ & Solvias & 4 & 46.4 \\
\hline L175 & SL-J013-1 & $\begin{array}{l}\text { (R)-1-[(SP)-2-[Bis(4-methoxy-3,5- } \\
\text { dimethylphenyl)phosphino]ferrocenyl\}ethyldi-tert-butylphosphine }\end{array}$ & Solvias & 97 & -2.5 \\
\hline L176 & SL-J212-1 & (R)-1-[(SP)-2-(di(2-furyl)phosphino)ferrocenyl]ethyldi-tert-butylphosphine & Solvias & 84 & -49.3 \\
\hline L177 & SL-J417-1 & $\begin{array}{l}\text { (R)-1-\{(SP)-2-[Bis[3,5-bis2-methylphenyl]phosphino]ferrocenyl\}ethyldi(3,5- } \\
\text { xylyl)phosphine }\end{array}$ & Solvias & 10 & 47.6 \\
\hline L178 & SL-J425-1 & $\begin{array}{l}\text { (S)-1-\{(RP)-2-[Bis(4-methoxy-3,5-dimethylphenyl)phosphino]ferrocenyl\}- } \\
\text { ethylbis(2-methylphenyl)phosphine }\end{array}$ & Solvias & 5 & 7.6 \\
\hline L179 & SL-J452-1 & $\begin{array}{l}\text { (R)-1-\{(SP)-2-[Di(2-furyl)phosphino]ferrocenyl\}ethylbis(2- } \\
\text { methylphenyl)phosphine }\end{array}$ & Solvias & 8 & 2.8 \\
\hline L180 & SL-J503-1 & $\begin{array}{l}\text { (R)-1-[(SP)-2-(Diethylphosphino)ferrocenyl]ethylbis }(2- \\
\text { methylphenyl)phosphine }\end{array}$ & Solvias & 7 & 20.1 \\
\hline L181 & SL-J210-1 & $\begin{array}{l}\text { (R)-1-\{(SP)-2-[Bis[3,5- } \\
\text { bis(trifluoromethyl)phenyl]phosphino]ferrocenyl\}ethyldi-tert-butylphosphine }\end{array}$ & Solvias & 4 & -1.2 \\
\hline L182 & SL-J003-1 & (R)-1-[(SP)-2-(Dicyclohexylphosphino)ferrocenyl]ethyldicyclohexylphosphine & Solvias & 41 & -37.2 \\
\hline L183 & SL-J110-1 & $\begin{array}{l}\text { (R)-1-[(SP)-2-(Diphenylphosphino)ferrocenyl]ethylbis(4- } \\
\text { (trifluoromethyl)phenyl)phosphine }\end{array}$ & Solvias & 0 & - \\
\hline L184 & SL-J007-1 & $\begin{array}{l}\text { (R)-1-\{(SP)-2-[Bis(4-methoxy-3,5- } \\
\text { dimethylphenyl)phosphino]ferrocenyl\}ethyldicyclohexylphosphine }\end{array}$ & Solvias & 11 & 49.6 \\
\hline L185 & SL-J009-1 & (R)-1-[(SP)-2-(Dicyclohexylphosphino)ferrocenyl]ethyldi-tert-butylphosphine & Solvias & 96 & -83.8 \\
\hline L186 & SL-J012-1 & $\begin{array}{l}\text { (R)-1-\{(SP)-2-[Bis[4-methylphenyl]phosphino]ferrocenyl\}ethyldi-tert- } \\
\text { butylphosphine }\end{array}$ & Solvias & 94 & 13.3 \\
\hline L187 & SL-J015-2 & (S)-1-\{(RP)-2-[Di(2-furyl)phosphino]ferrocenyl\}ethyldi(3,5-xylyl)phosphine & Solvias & 7 & 59.5 \\
\hline L188 & SL-J418-1 & $\begin{array}{l}\text { (R)-1-\{(SP)-2-[Bis(4-methoxy-3,5-dimethylphenyl)phosphino]ferrocenyl\}- } \\
\text { ethyldi(3,5-xylyl)phosphine }\end{array}$ & Solvias & 5 & 2.8 \\
\hline L189 & SL-J504-1 & $\begin{array}{l}\text { (R)-1-[(SP)-2-(Dicyclohexylphosphino)ferrocenyl]ethylbis(2- } \\
\text { methylphenyl)phosphine }\end{array}$ & Solvias & 11 & -45 \\
\hline L190 & SL-J605-1 & $\begin{array}{l}\text { (R)-1-[(SP)-2-((R)- } \\
\text { methylphenylphosphino)ferrocenyl] ethyldicyclohexylphosphine }\end{array}$ & Solvias & 5 & 53.5 \\
\hline
\end{tabular}




\begin{tabular}{|l|l|l|l|l|l|}
\hline L191 & SL-J507-1 & (R)-1-[(SP)-2-(Diethylphosphino)ferrocenyl]ethyldi(3,5-xyly)phosphine & Solvias & 10 & -58.7 \\
\hline L192 & SL-J034-1 & $\begin{array}{l}\text { (R)-1-[(SP)-2-(Diphenylphosphino)-3- } \\
\text { trimethylsilylferrocenyl]ethyldicyclohexylphosphine }\end{array}$ & Solvias & 37 & -77.1 \\
\hline
\end{tabular}

\section{Solvent Screen}

15 solvents $\mathbf{X} 3$ best Walphos ligands $\mathbf{X} 2$ loadings according to the general procedure with the following conditions: $\mathrm{X}$ mol\% Ru, 1.1(X) mol\% ligand, solvent (0.02 M), $\mathrm{H}_{2}(500 \mathrm{psi}), 50{ }^{\circ} \mathrm{C}, 14$ hours.

$\%$ conversion

Ligand / Ru

\begin{tabular}{|c|c|c|c|c|c|c|c|}
\hline & & W-022-1 & W-022-1 & W-017-1 & W-017-1 & W-003-1 & W-003-1 \\
\hline & & $10 \mathrm{~mol} \%$ & $1 \mathrm{~mol} \%$ & $10 \mathrm{~mol} \%$ & $1 \mathrm{~mol} \%$ & $10 \mathrm{~mol} \%$ & $1 \mathrm{~mol} \%$ \\
\hline & $\mathrm{MeOH}$ & 100.0 & 100.0 & 100.0 & 100.0 & 100.0 & 100.0 \\
\hline & iPrOH & 100.0 & 99.4 & 99.4 & 71.7 & 100.0 & 100.0 \\
\hline & $\mathrm{EtOH}$ & 100.0 & 100.0 & 100.0 & 100.0 & 100.0 & 100.0 \\
\hline & 2-Me THF & 100.0 & 100.0 & 99.3 & 4.6 & 100.0 & 100.0 \\
\hline & CPME & 99.3 & 64.0 & 98.9 & 1.2 & 100.0 & 100.0 \\
\hline & TFE & 100.0 & 100.0 & 99.3 & 92.6 & 100.0 & 45.6 \\
\hline & DME & 99.3 & 61.8 & 99.1 & 1.1 & 100.0 & 87.0 \\
\hline Solvent & iPAc & 100.0 & 80.0 & 98.8 & 3.6 & 100.0 & 100.0 \\
\hline & EtOAc & 99.1 & 100.0 & 99.0 & 4.5 & 100.0 & 100.0 \\
\hline & MEK & 100.0 & 100.0 & 99.2 & 8.7 & 100.0 & 96.9 \\
\hline & MIBK & 100.0 & 100.0 & 99.2 & 24.6 & 100.0 & 100.0 \\
\hline & Tol & 100.0 & 24.8 & 100.0 & 0.0 & 84.3 & 0.0 \\
\hline & $\mathrm{PhCl}$ & 100.0 & 38.5 & 100.0 & 0.0 & 100.0 & 71.8 \\
\hline & DCE & 99.1 & 13.2 & 98.6 & 5.0 & 99.1 & 44.5 \\
\hline & $\mathrm{CF}_{3} \mathrm{Tol}$ & 100.0 & 16.8 & 100.0 & 3.8 & 100.0 & 37.6 \\
\hline
\end{tabular}

$\%$ ee

Ligand / Ru

\begin{tabular}{|c|c|c|c|c|c|c|c|}
\hline & & W-022-1 & W-022-1 & W-017-1 & W-017-1 & W-003-1 & W-003-1 \\
\hline & & $10 \mathrm{~mol} \%$ & $1 \mathrm{~mol} \%$ & $10 \mathrm{~mol} \%$ & $1 \mathrm{~mol} \%$ & $10 \mathrm{~mol} \%$ & $1 \mathrm{~mol} \%$ \\
\hline & $\mathrm{MeOH}$ & 97.0 & 93.1 & 96.6 & 94.3 & 92.4 & 55.6 \\
\hline & iPrOH & 95.9 & 88.7 & 96.0 & 87.4 & 71.3 & 44.3 \\
\hline & $\mathrm{EtOH}$ & 97.0 & 92.5 & 96.8 & 95.4 & 87.4 & 53.5 \\
\hline & 2-Me THF & 85.9 & 88.7 & 91.7 & 66.4 & 27.5 & 34.9 \\
\hline & CPME & 84.9 & 86.3 & 88.8 & 100.0 & 28.9 & 45.3 \\
\hline & TFE & 92.9 & 79.2 & 94.5 & 73.4 & 75.1 & 10.1 \\
\hline & DME & 82.6 & 81.8 & 81.8 & 100.0 & 18.3 & 26.7 \\
\hline Solvent & iPAc & 95.1 & 89.1 & 95.3 & 50.1 & 43.4 & 37.5 \\
\hline & EtOAc & 96.0 & 93.3 & 94.3 & 42.1 & 46.5 & 35.1 \\
\hline & MEK & 87.4 & 92.4 & 87.1 & 59.4 & 27.4 & 34.4 \\
\hline & MIBK & 98.6 & 96.8 & 100.0 & 79.9 & 91.2 & 85.2 \\
\hline & Tol & 89.4 & 100.0 & 89.1 & 0 & 43.4 & 0.0 \\
\hline & $\mathrm{PhCl}$ & 100.0 & 79.7 & 100.0 & 0.0 & 49.4 & 39.3 \\
\hline & DCE & 96.5 & 77.7 & 95.3 & 71.2 & 71.1 & 69.7 \\
\hline & CF3 Tol & 100.0 & 100.0 & 100.0 & 100.0 & 50.5 & 55.0 \\
\hline
\end{tabular}


Additional Catalyst Loading Study

$\mathrm{Ru} / \mathrm{SL}-W 022-1$ screened according to the general procedure (100 psi $\mathrm{H}_{2}, 60{ }^{\circ} \mathrm{C}$ for 14 hours):

\begin{tabular}{|c|c|c|c|}
\hline Solvent (0.4M) & mol\% & \% conversion & $\%$ ee \\
\hline MIBK & 0.50 & 100.0 & 98.0 \\
\hline MIBK & 0.40 & 100.0 & 97.9 \\
\hline MIBK & 0.35 & 100.0 & 98.0 \\
\hline MIBK & 0.30 & 100.0 & 97.9 \\
\hline MIBK & 0.25 & 97.4 & 97.6 \\
\hline MIBK & 0.20 & 98.7 & 97.1 \\
\hline $\mathrm{MeOH}$ & 0.50 & 100.0 & 93.9 \\
\hline $\mathrm{MeOH}$ & 0.40 & 100.0 & 93.9 \\
\hline $\mathrm{MeOH}$ & 0.35 & 100.0 & 94.0 \\
\hline $\mathrm{MeOH}$ & 0.30 & 100.0 & 94.3 \\
\hline $\mathrm{MeOH}$ & 0.25 & 100.0 & 93.5 \\
\hline
\end{tabular}




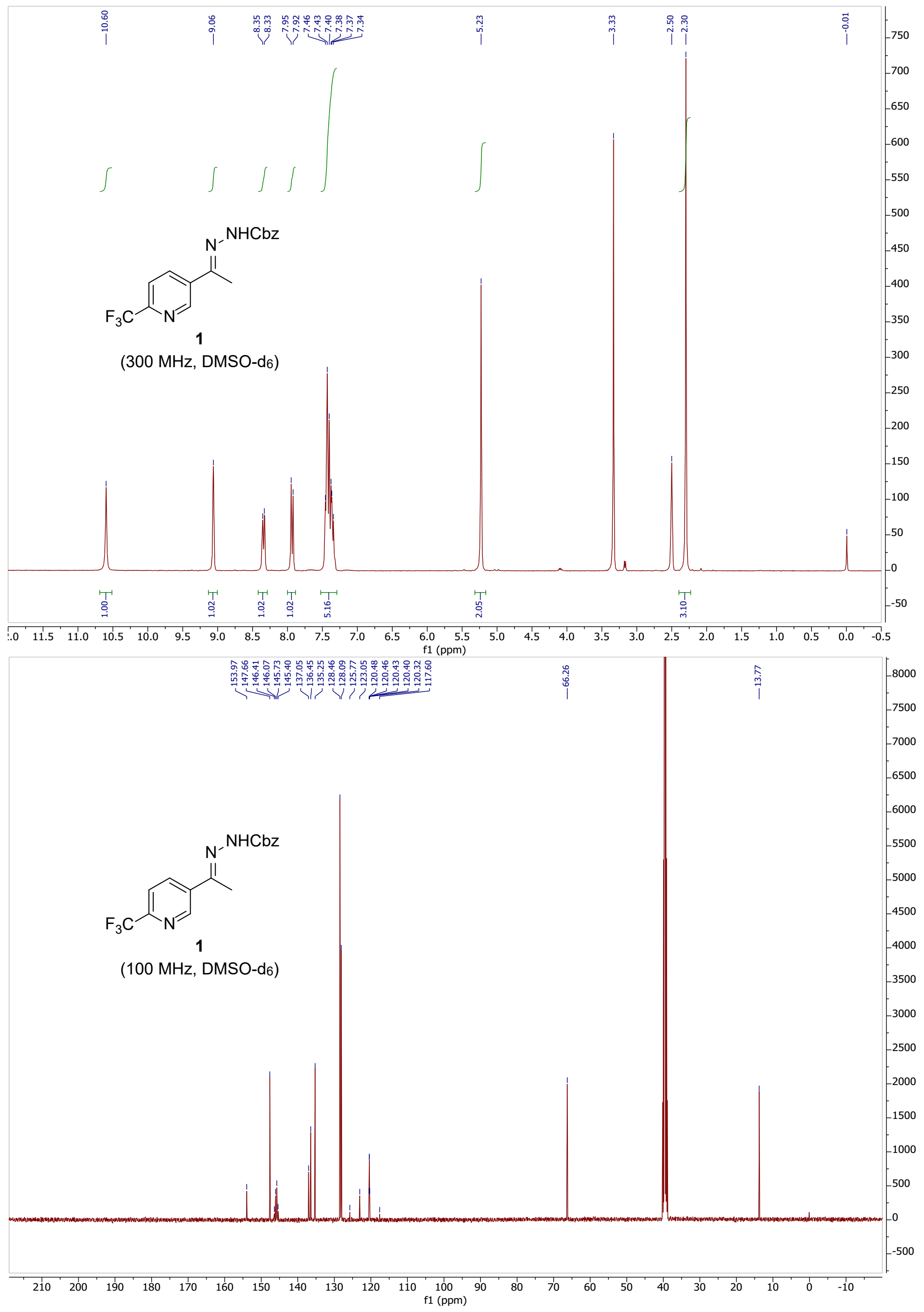




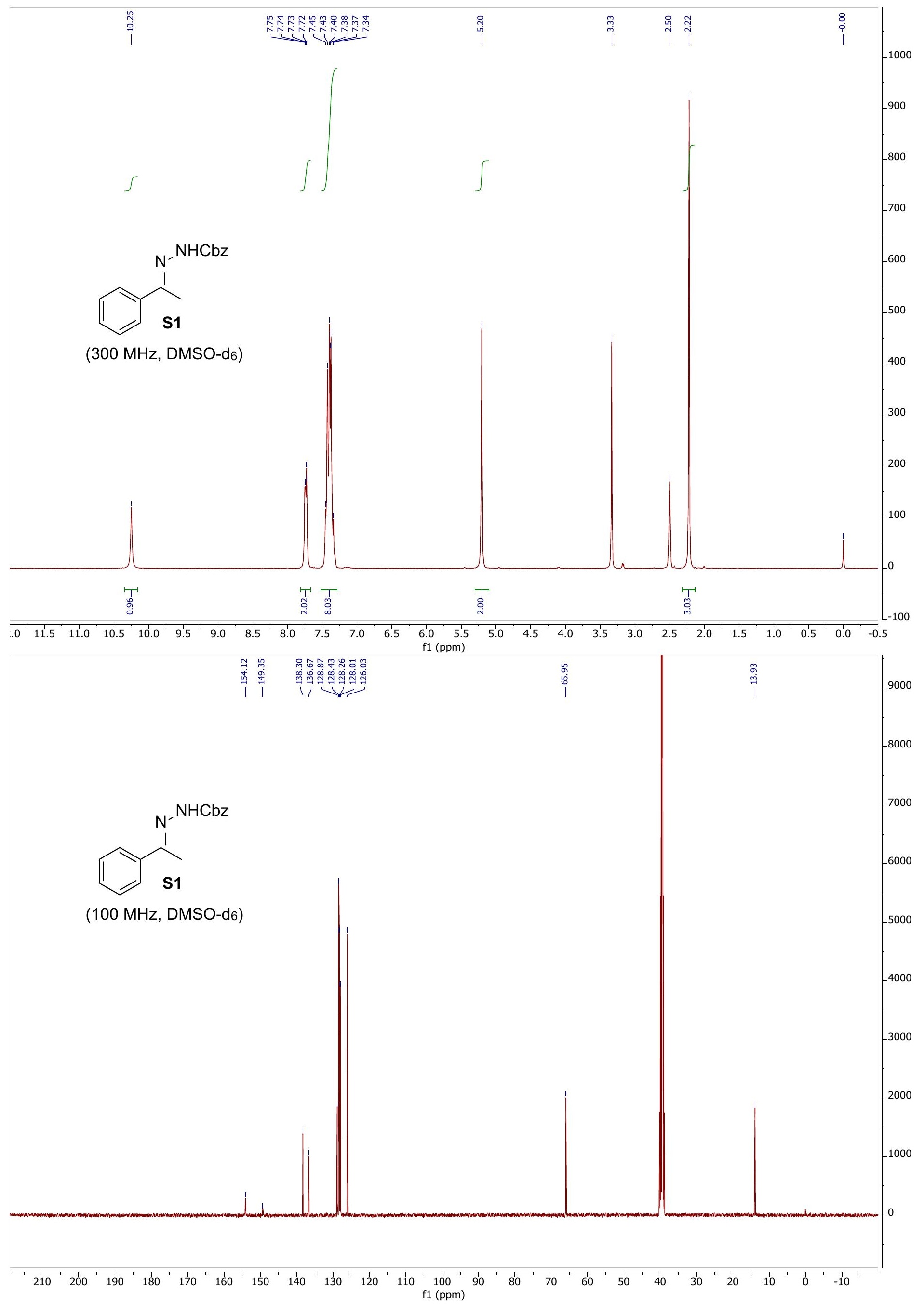




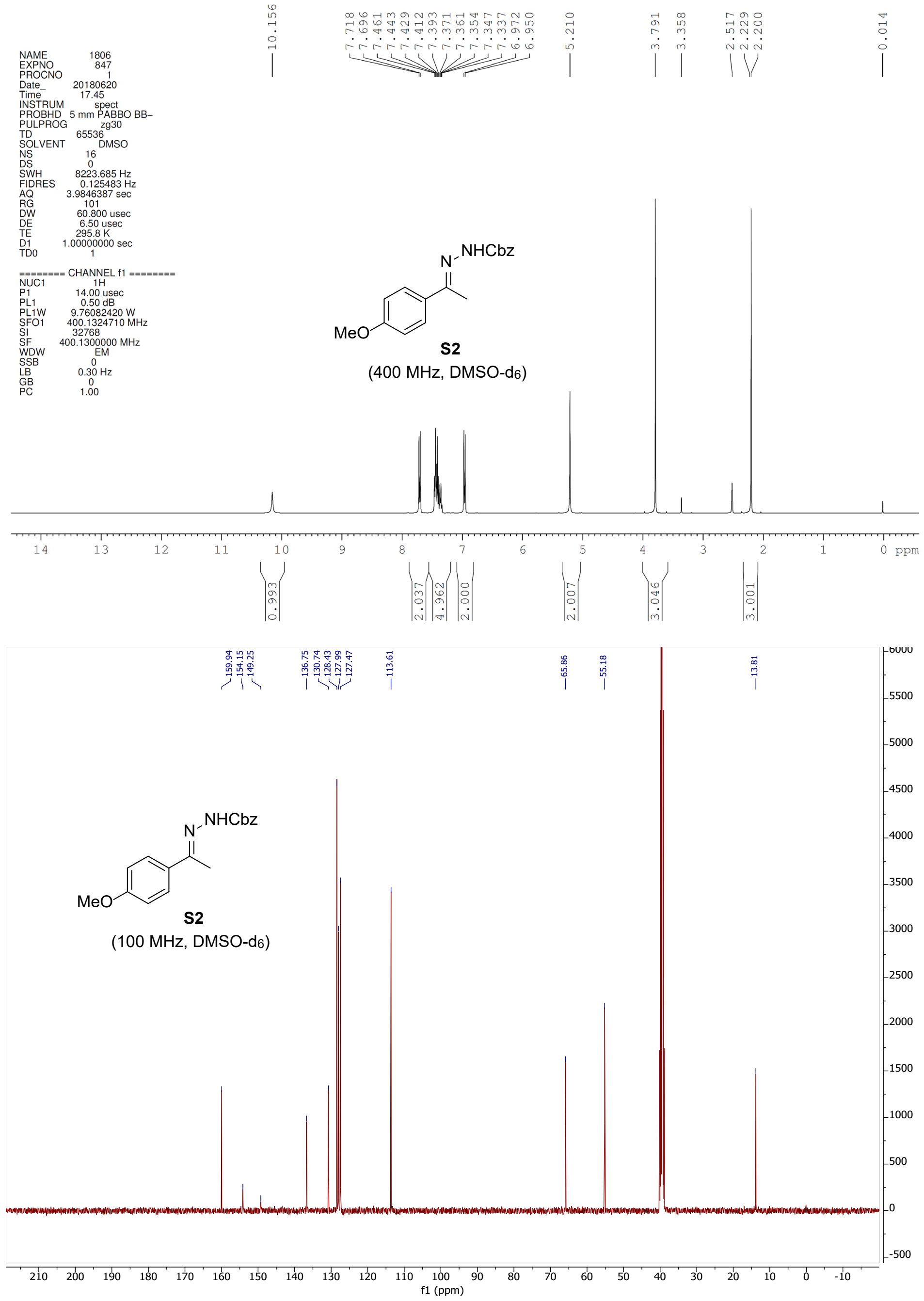




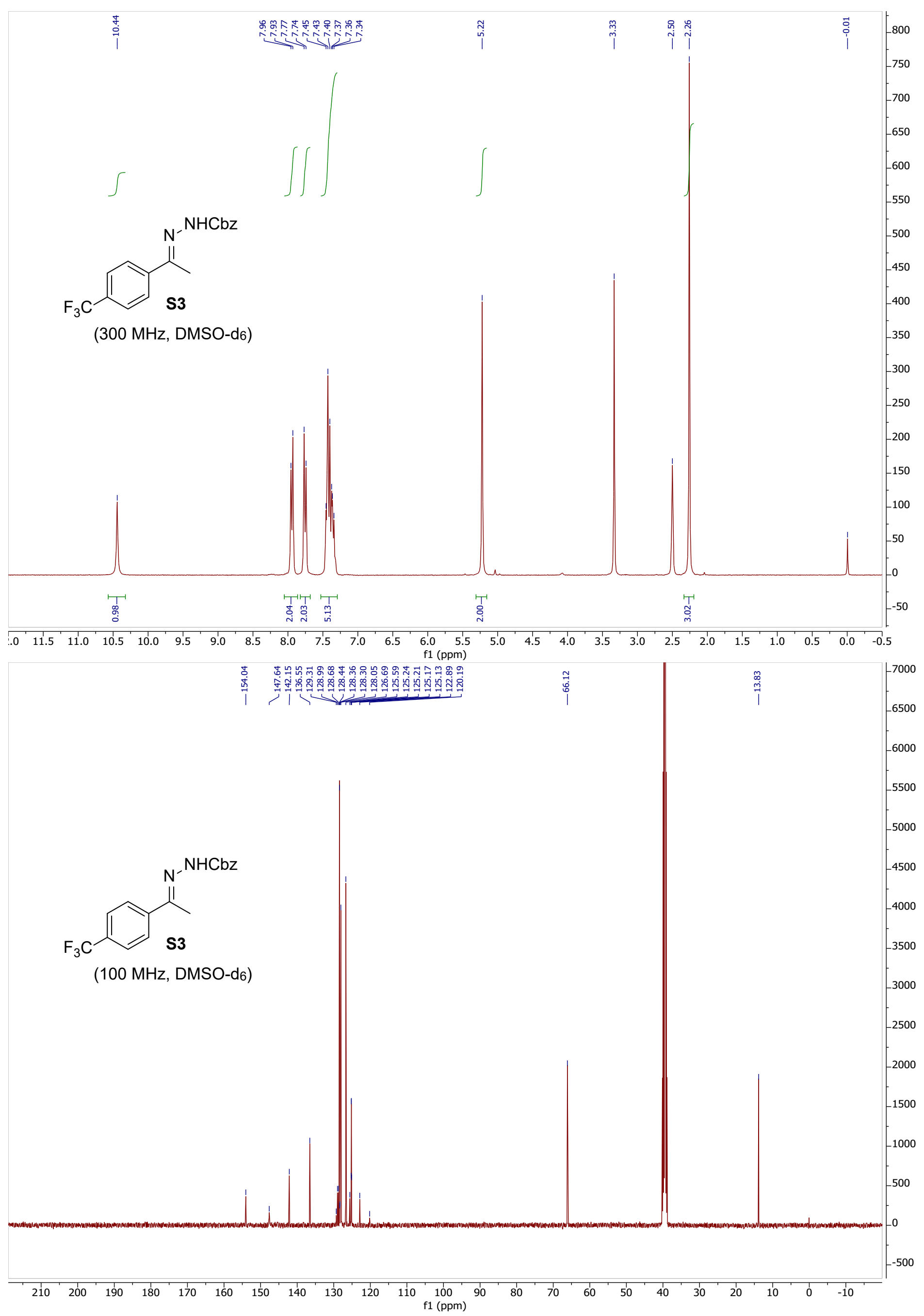




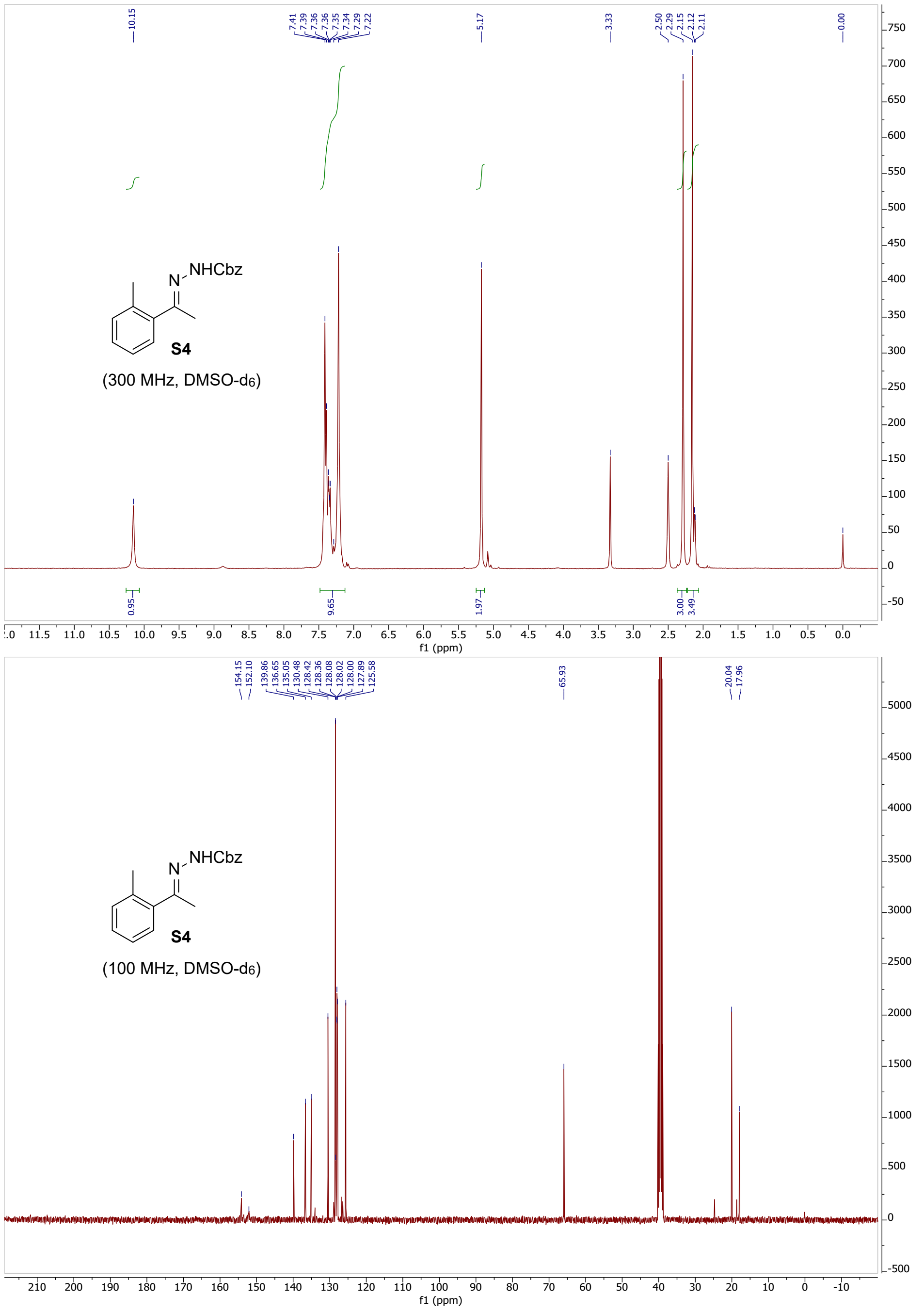




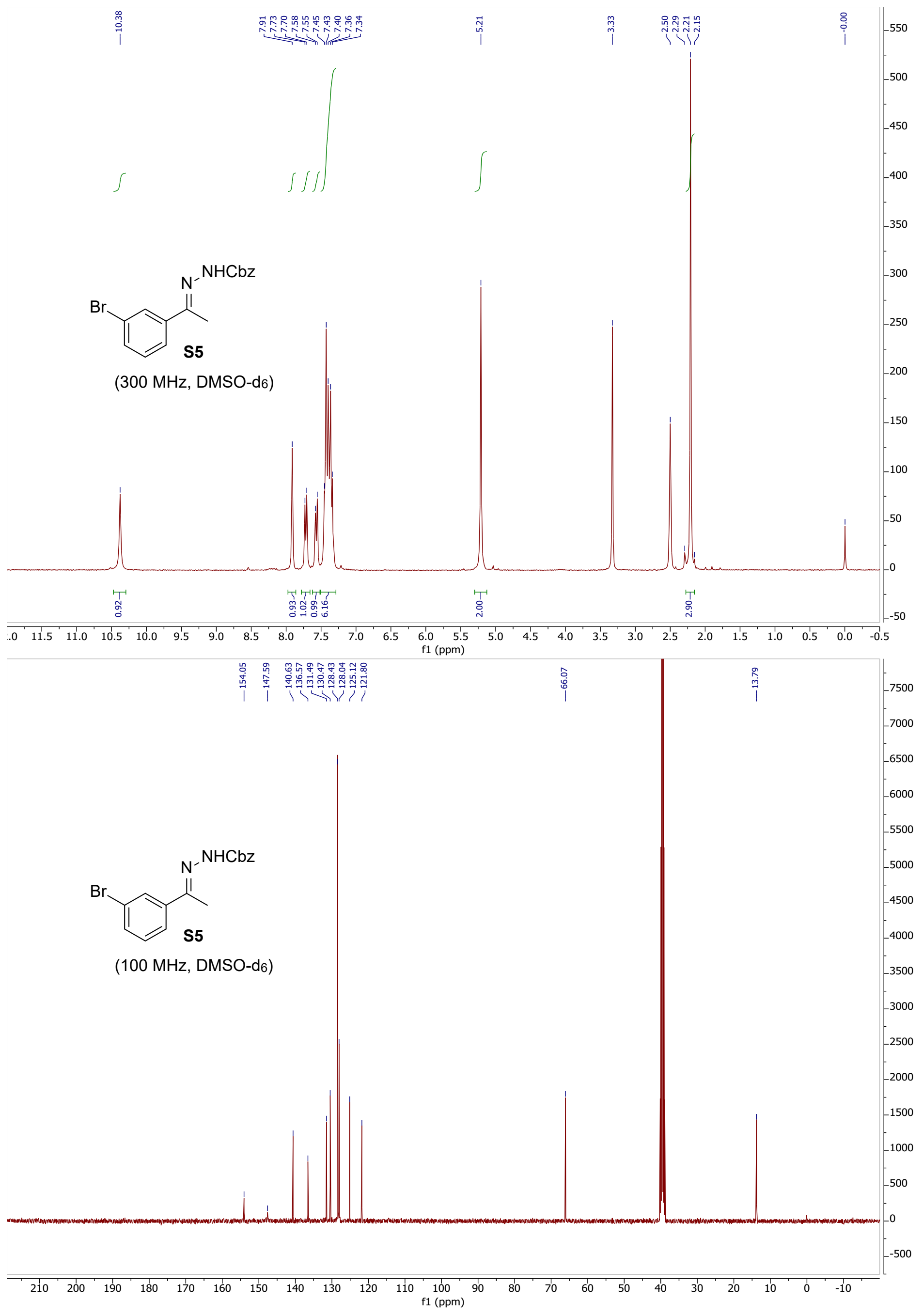




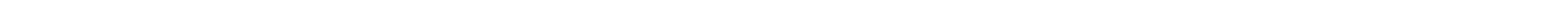




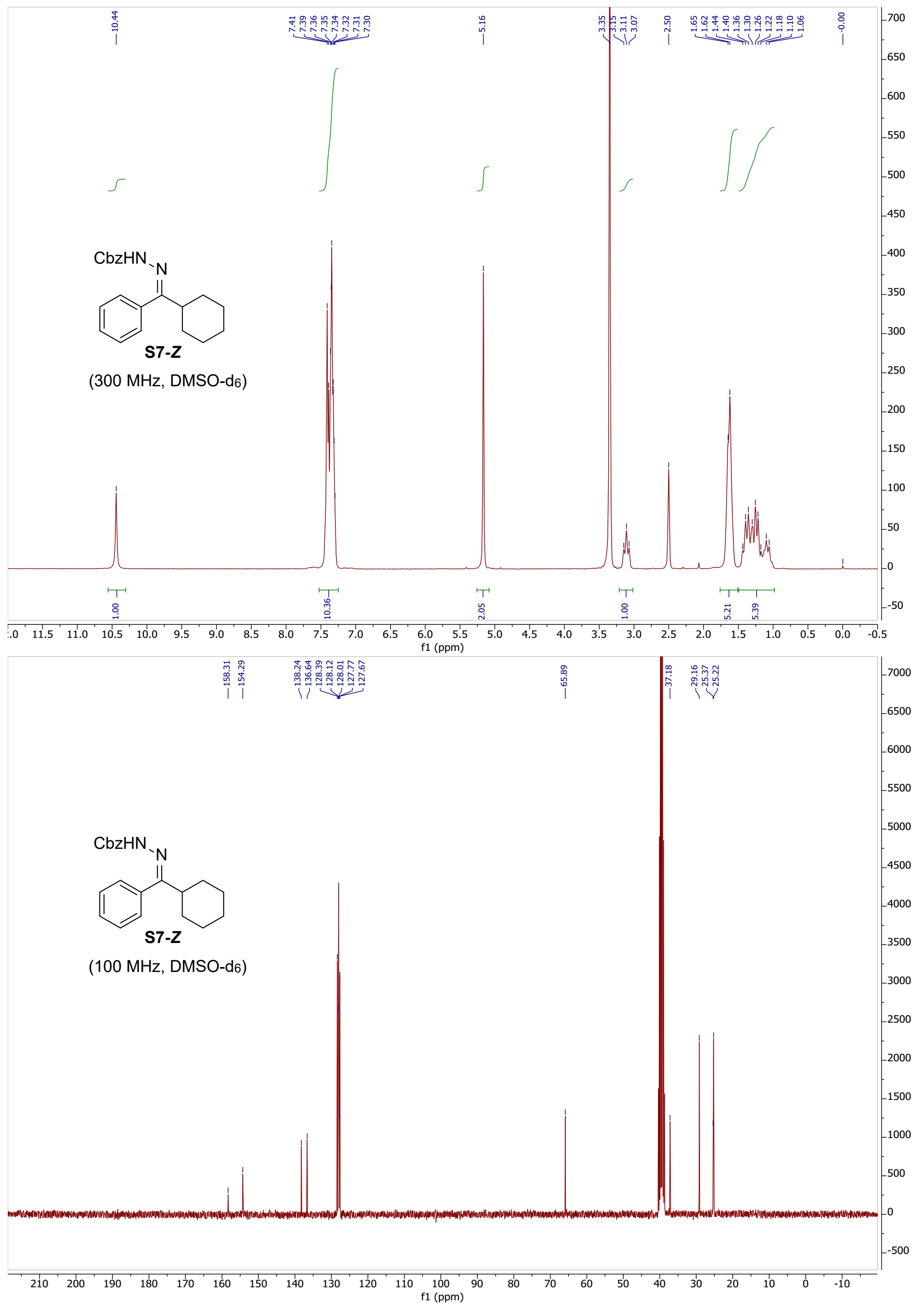




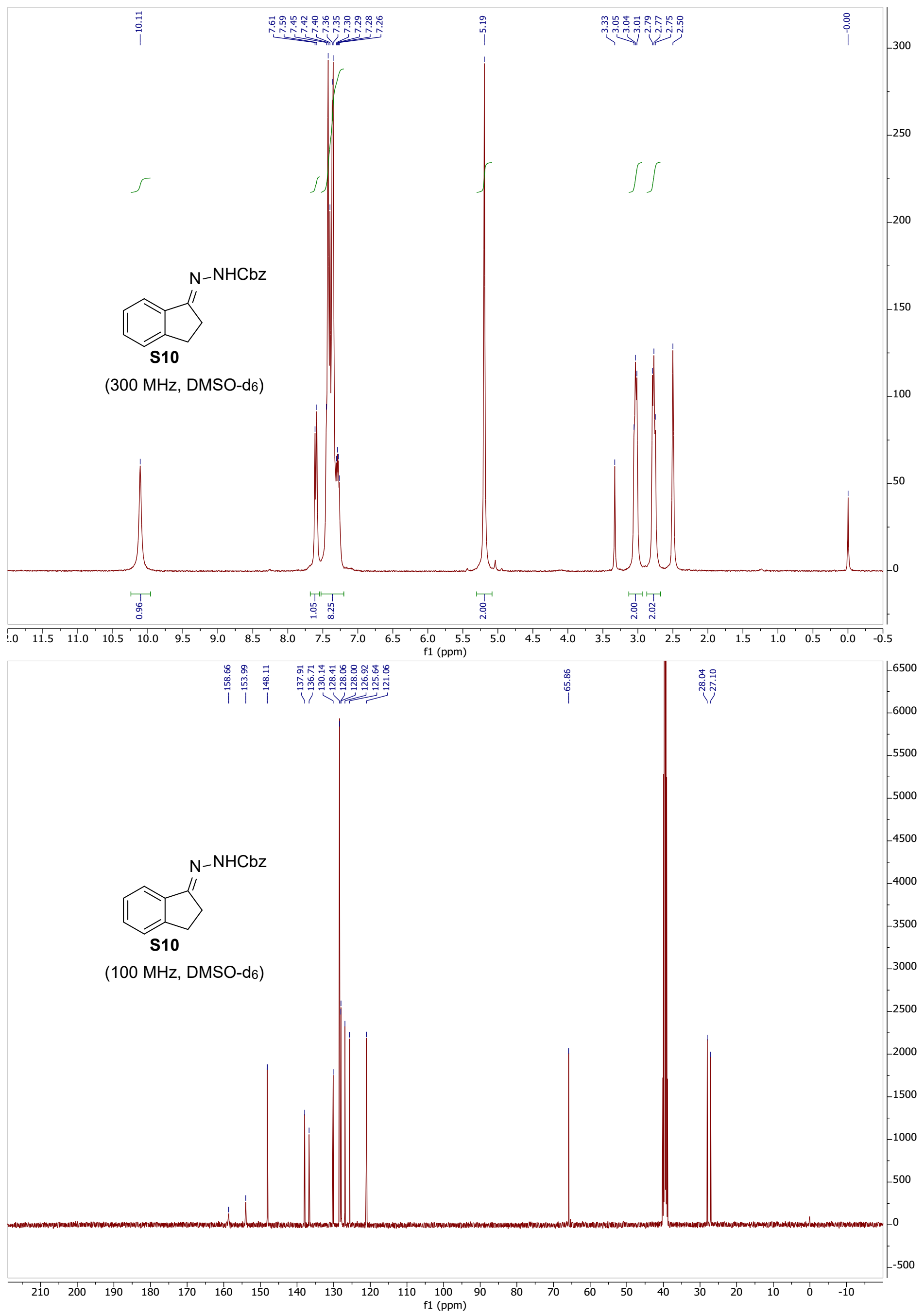




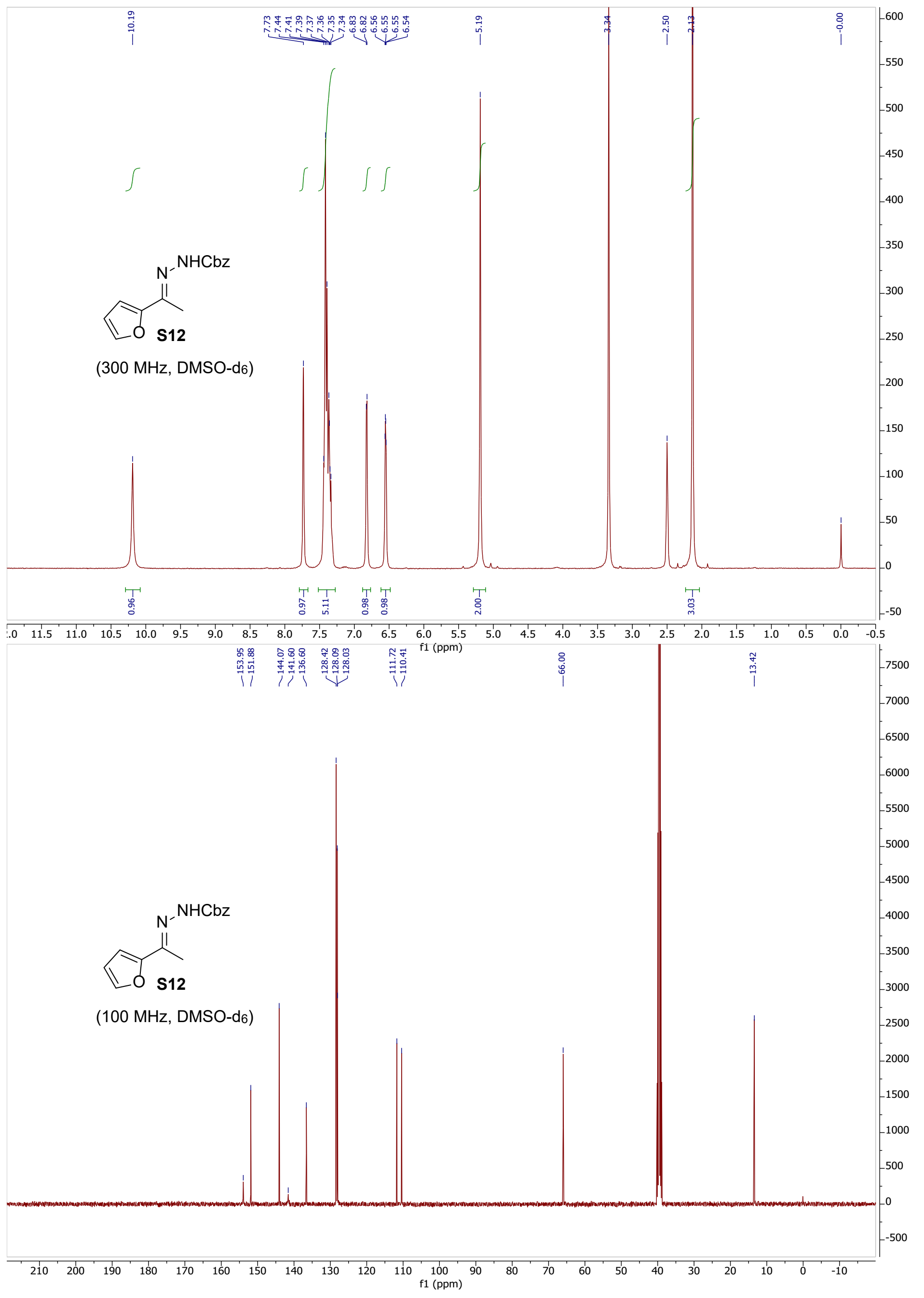




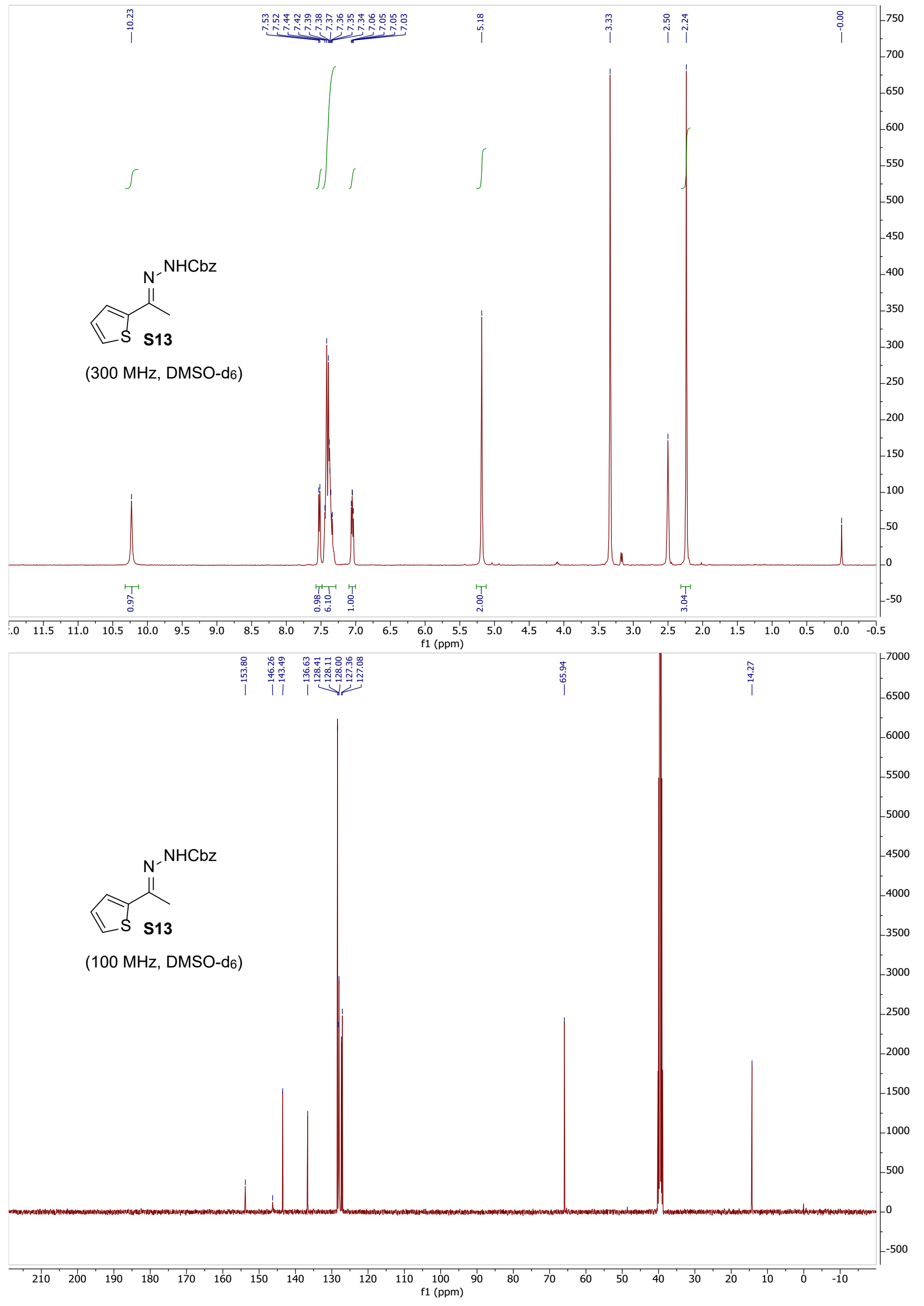




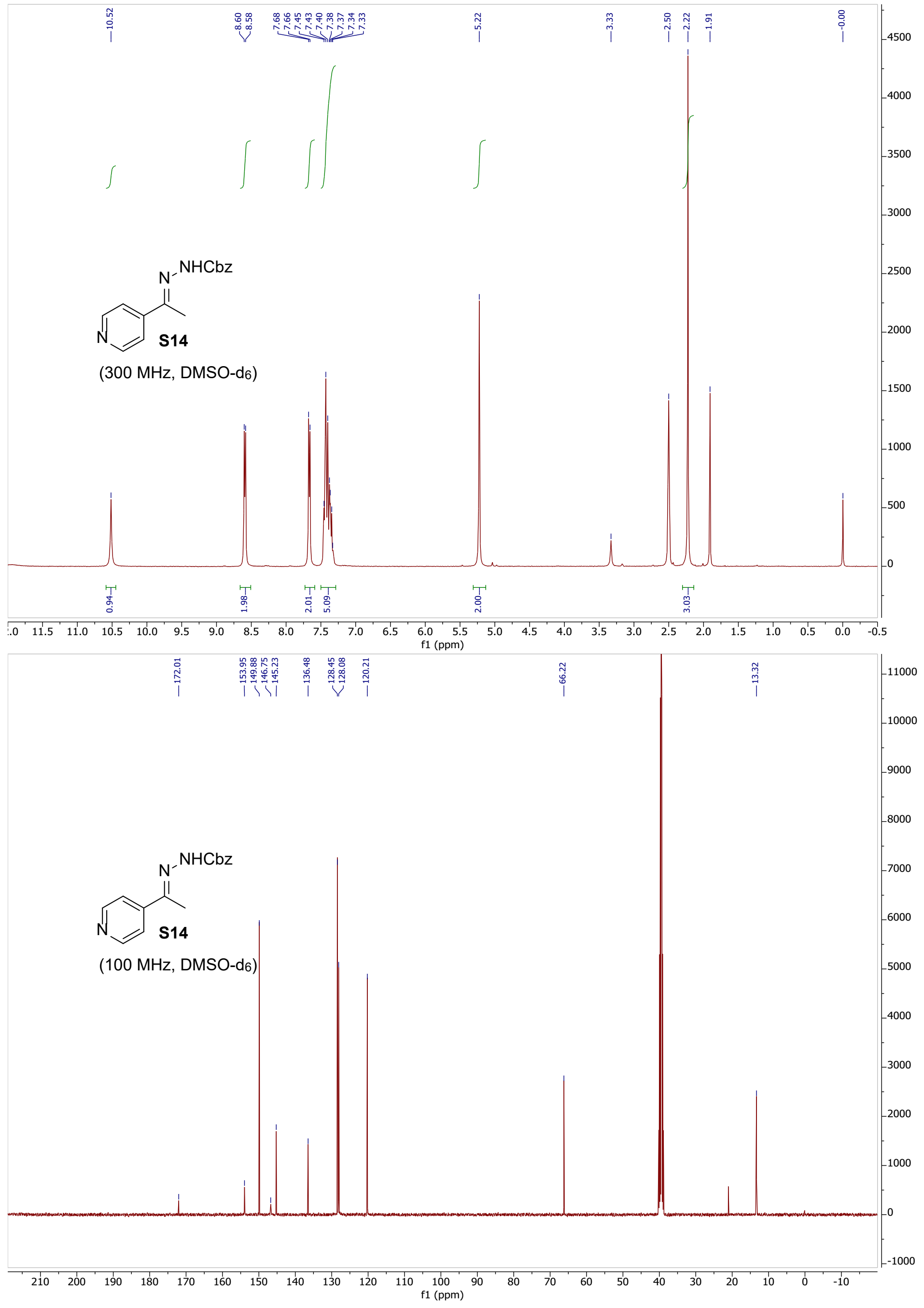




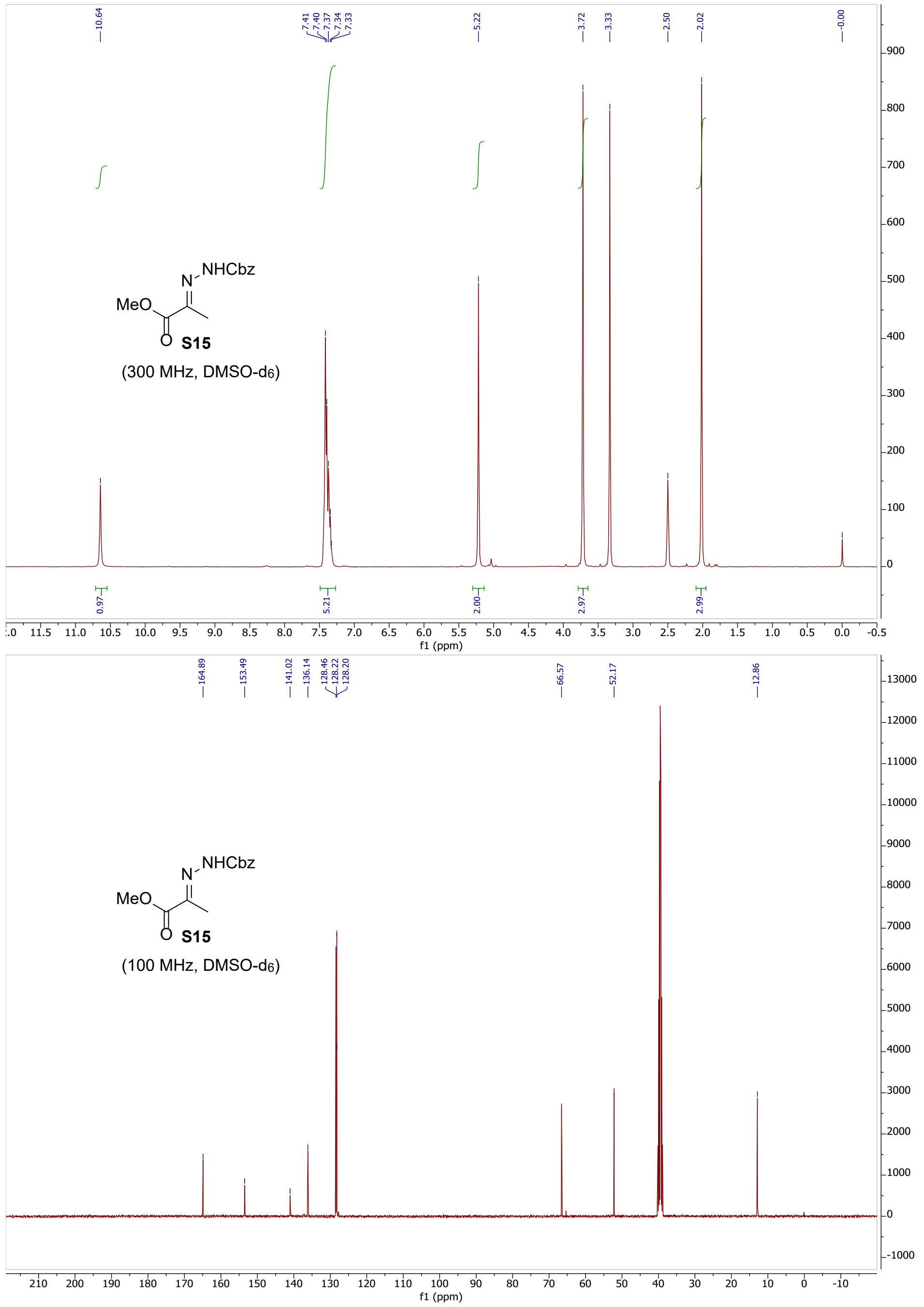




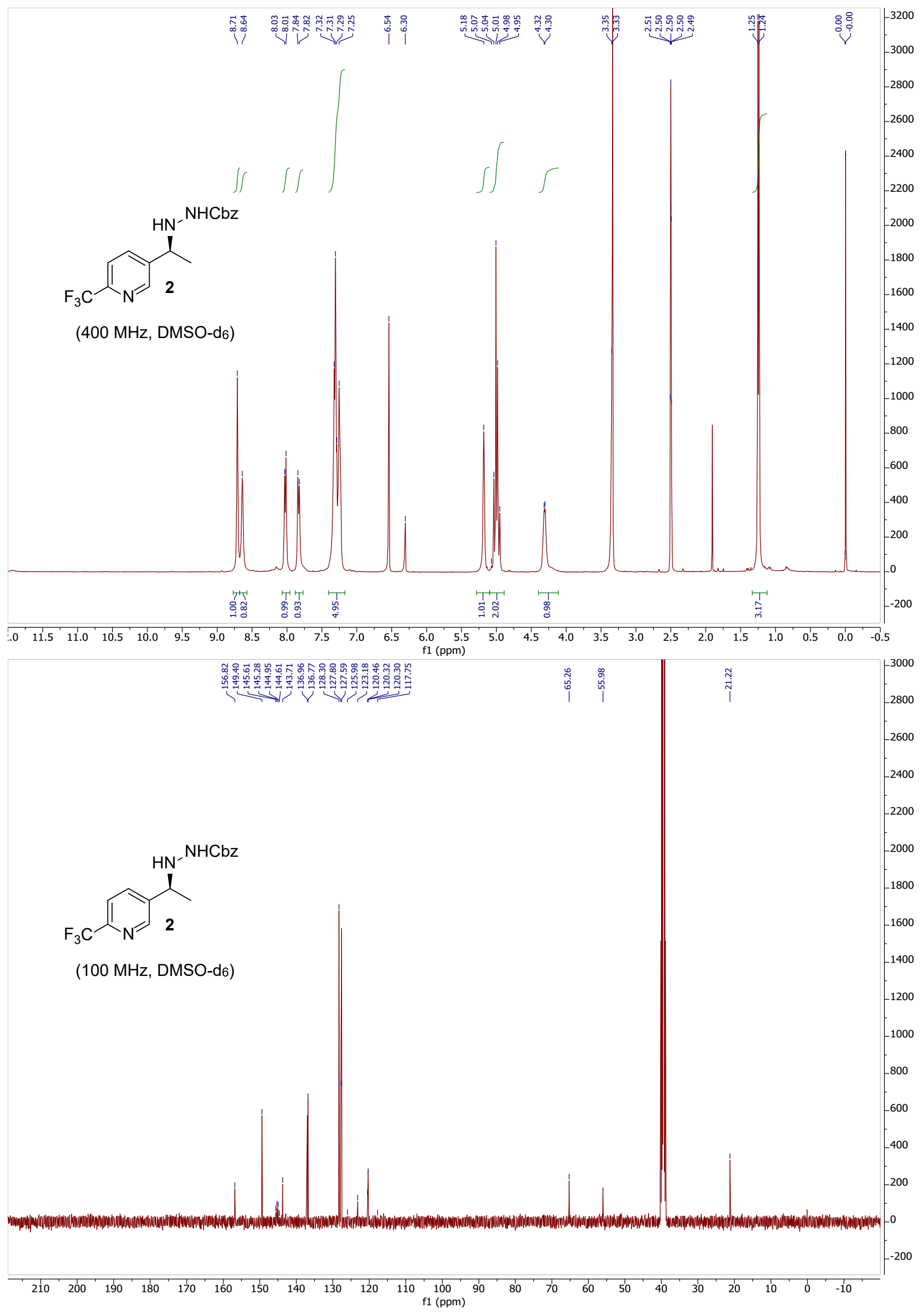




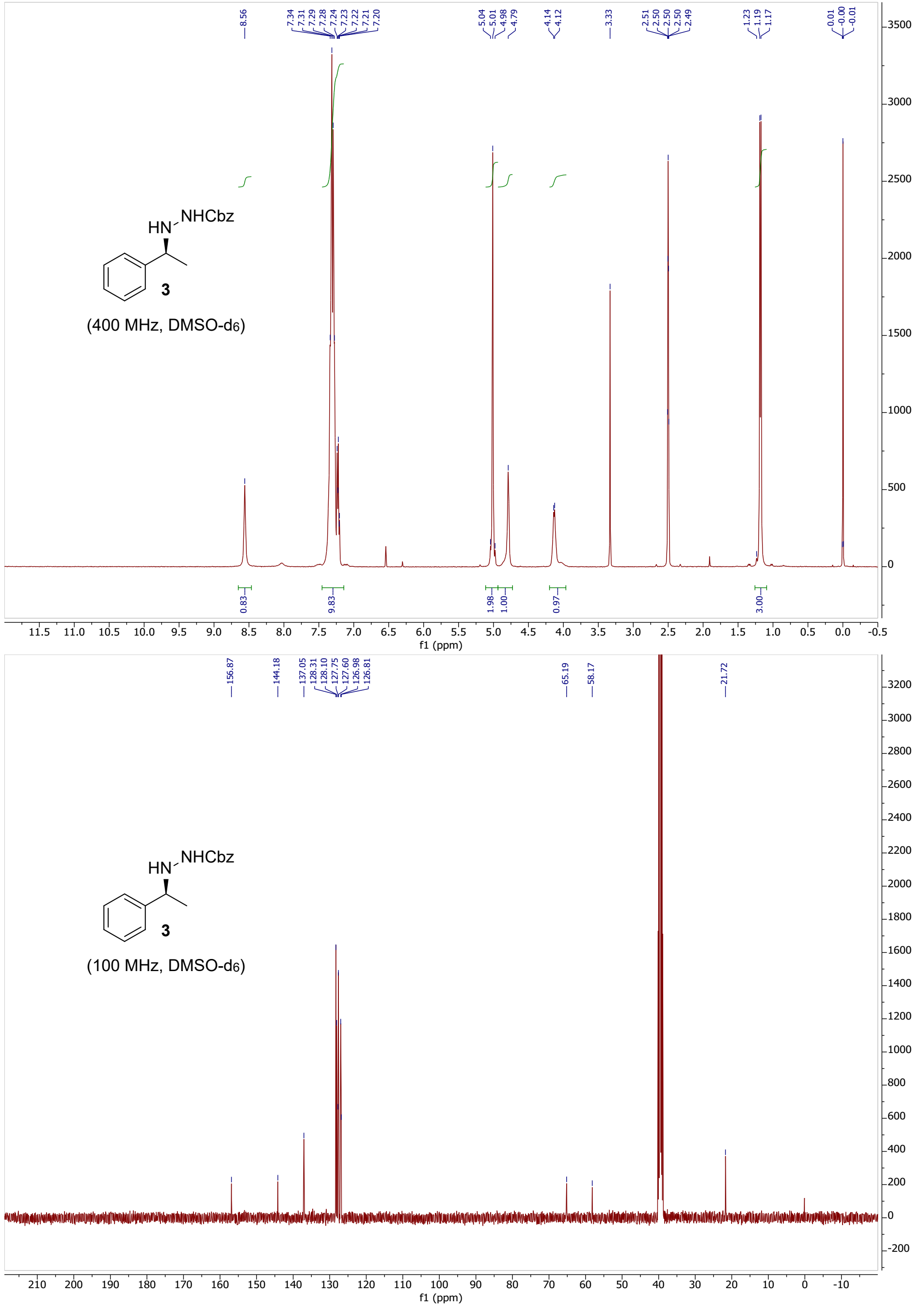




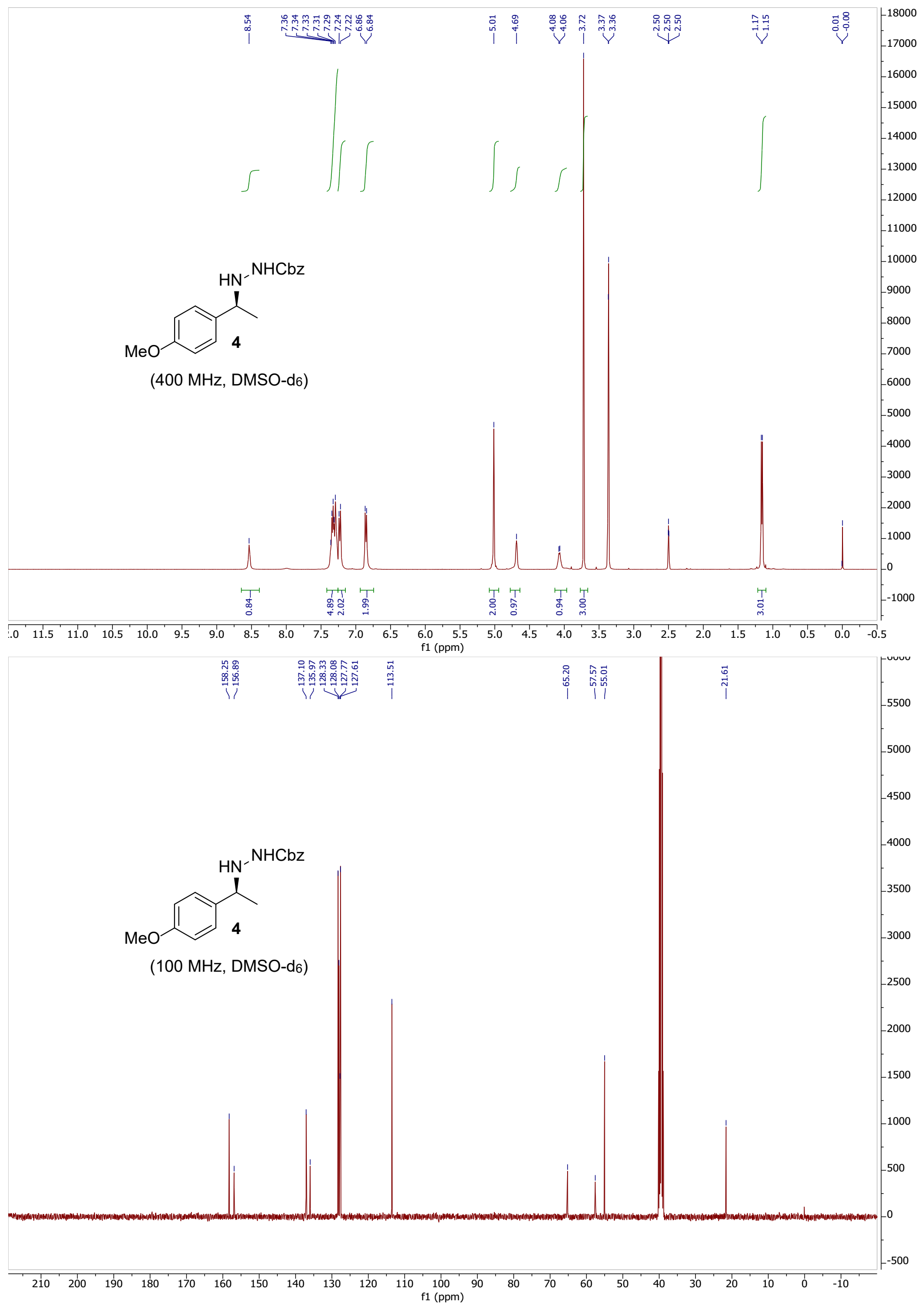




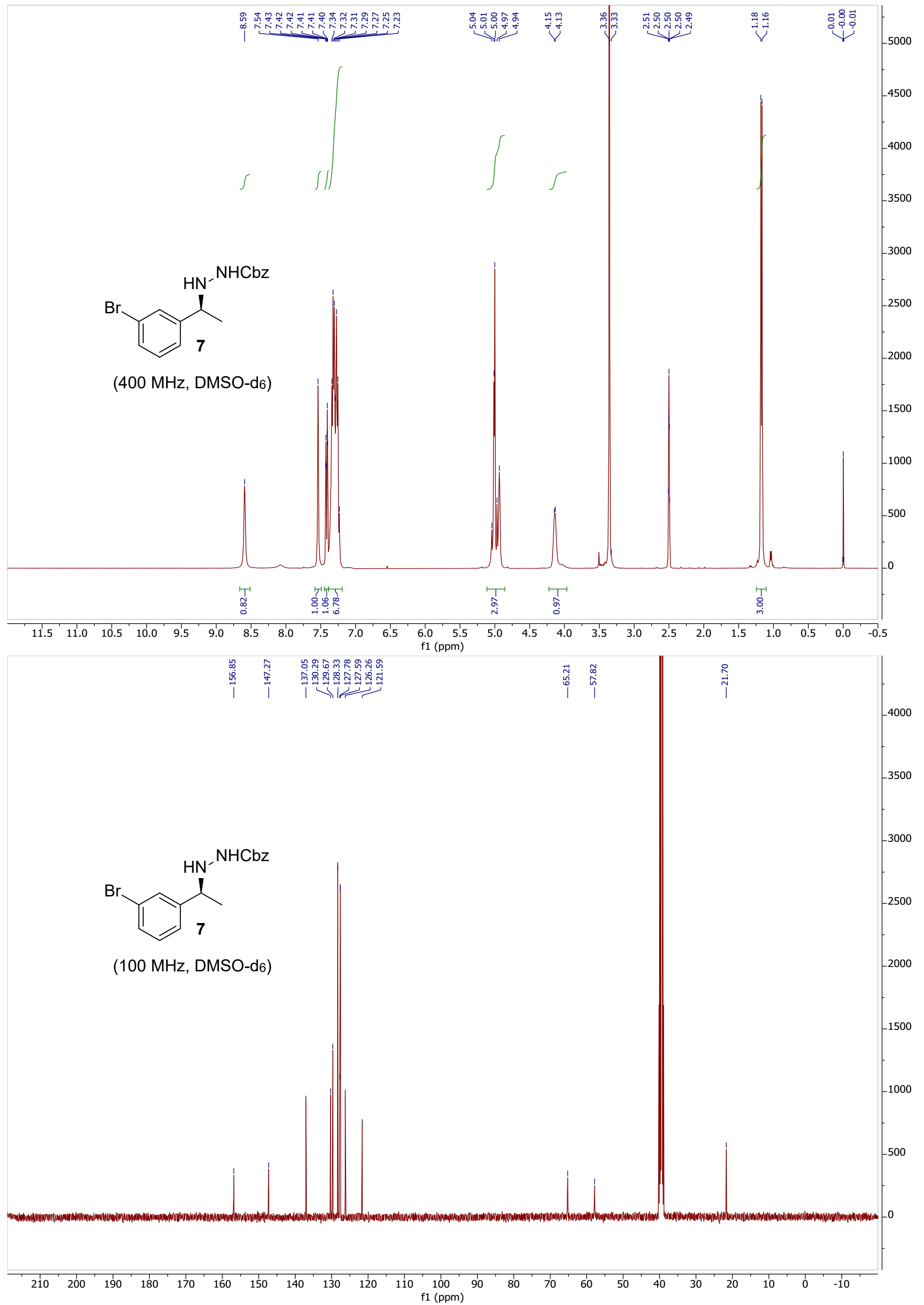




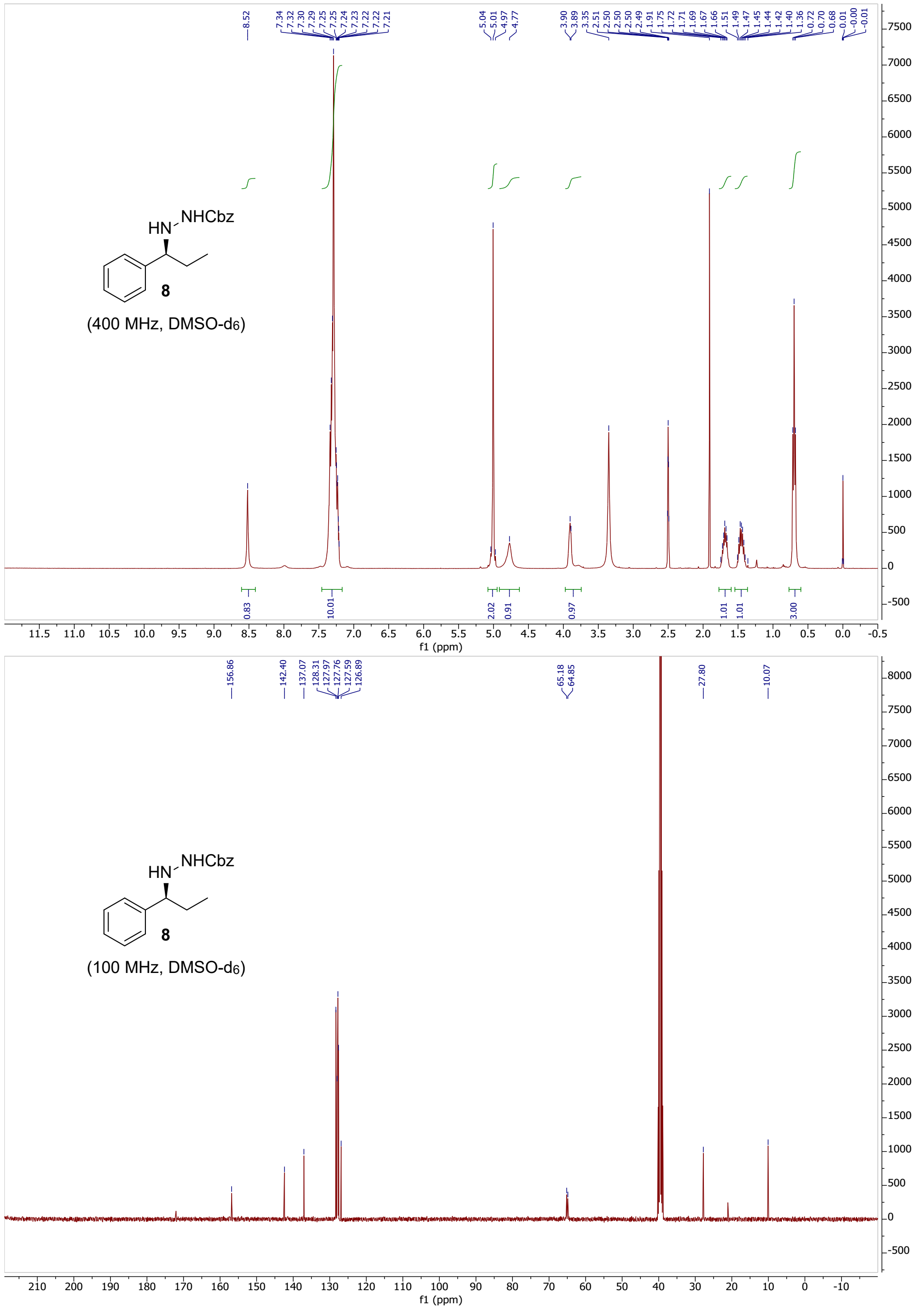




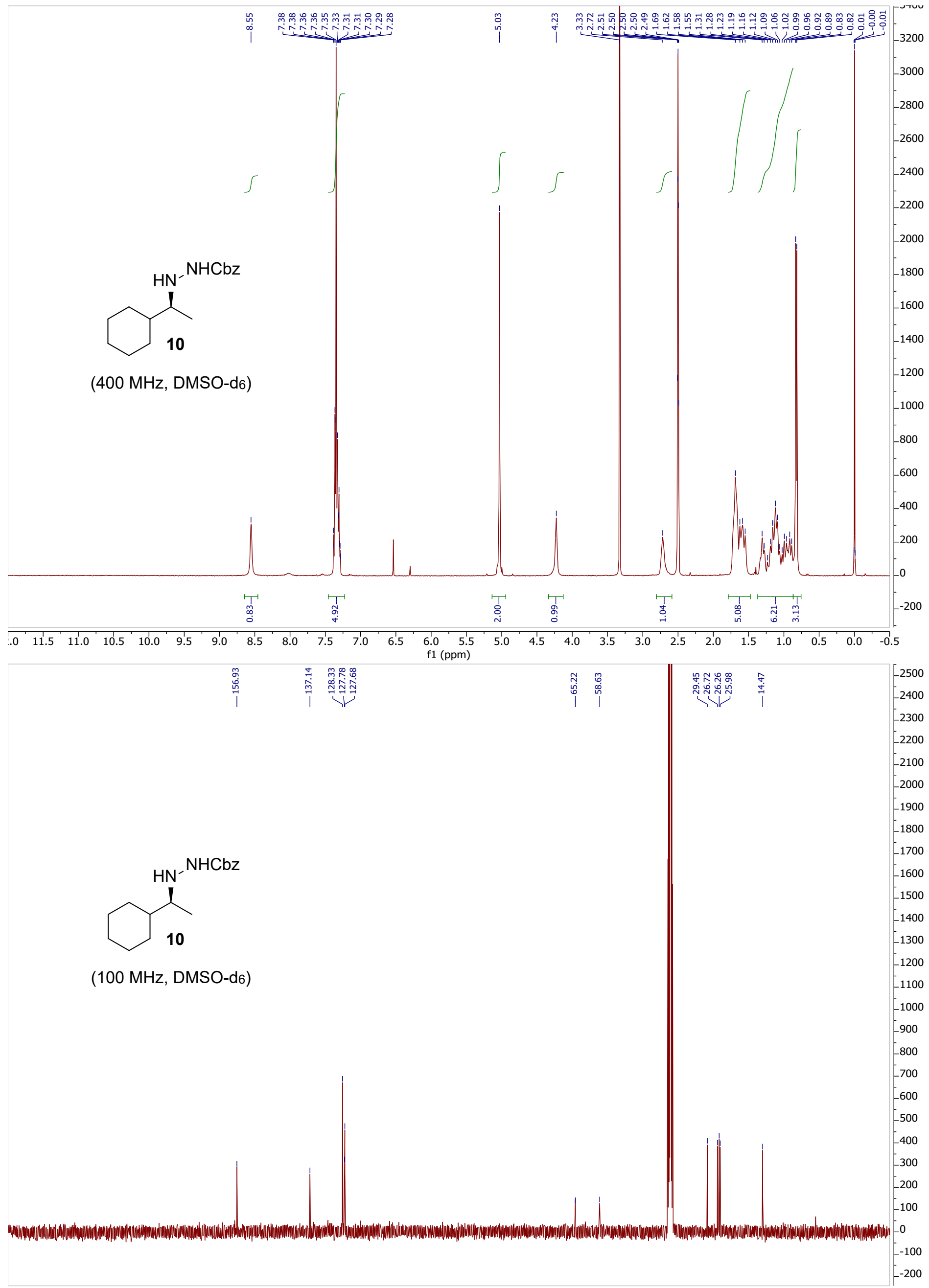




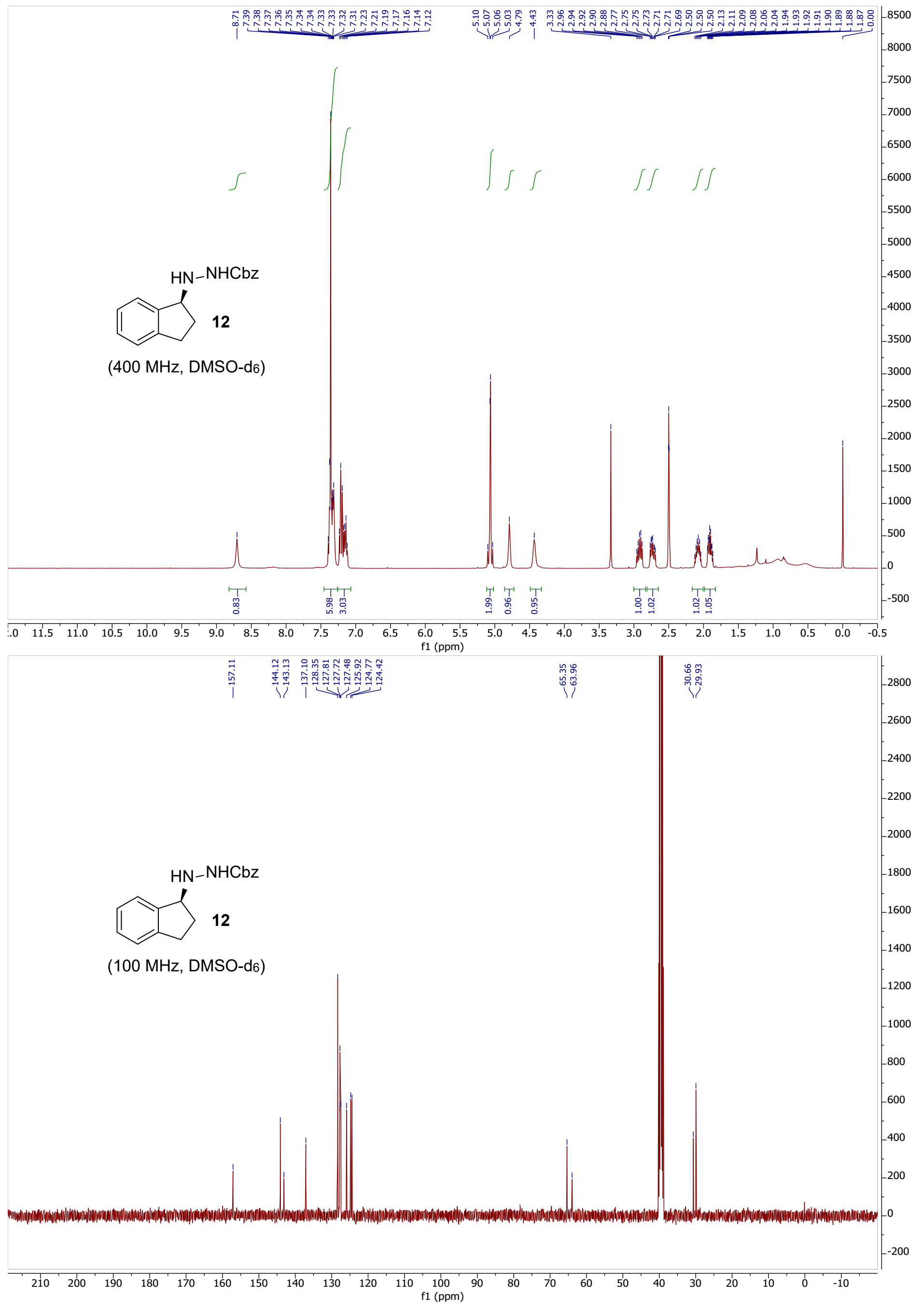




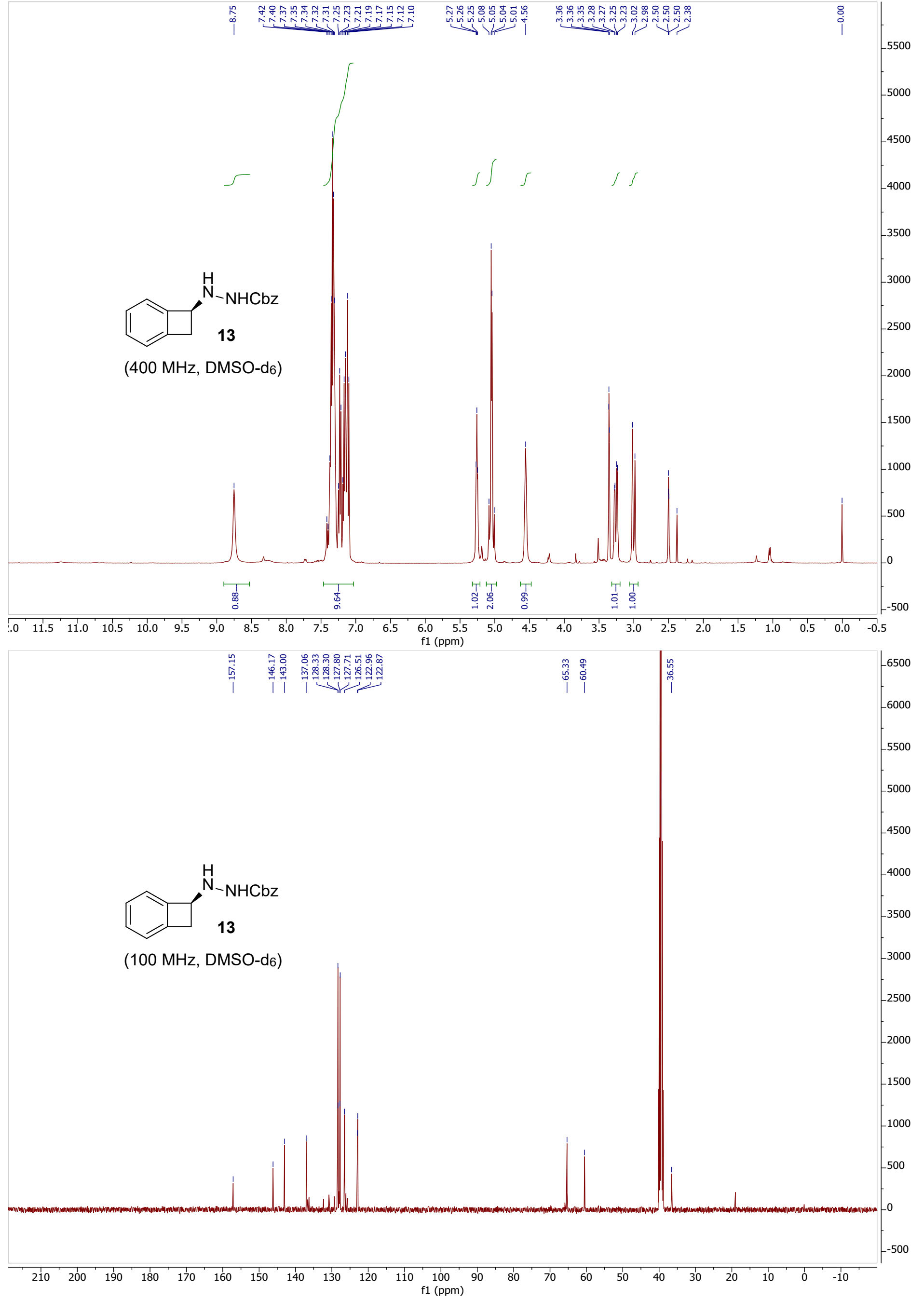




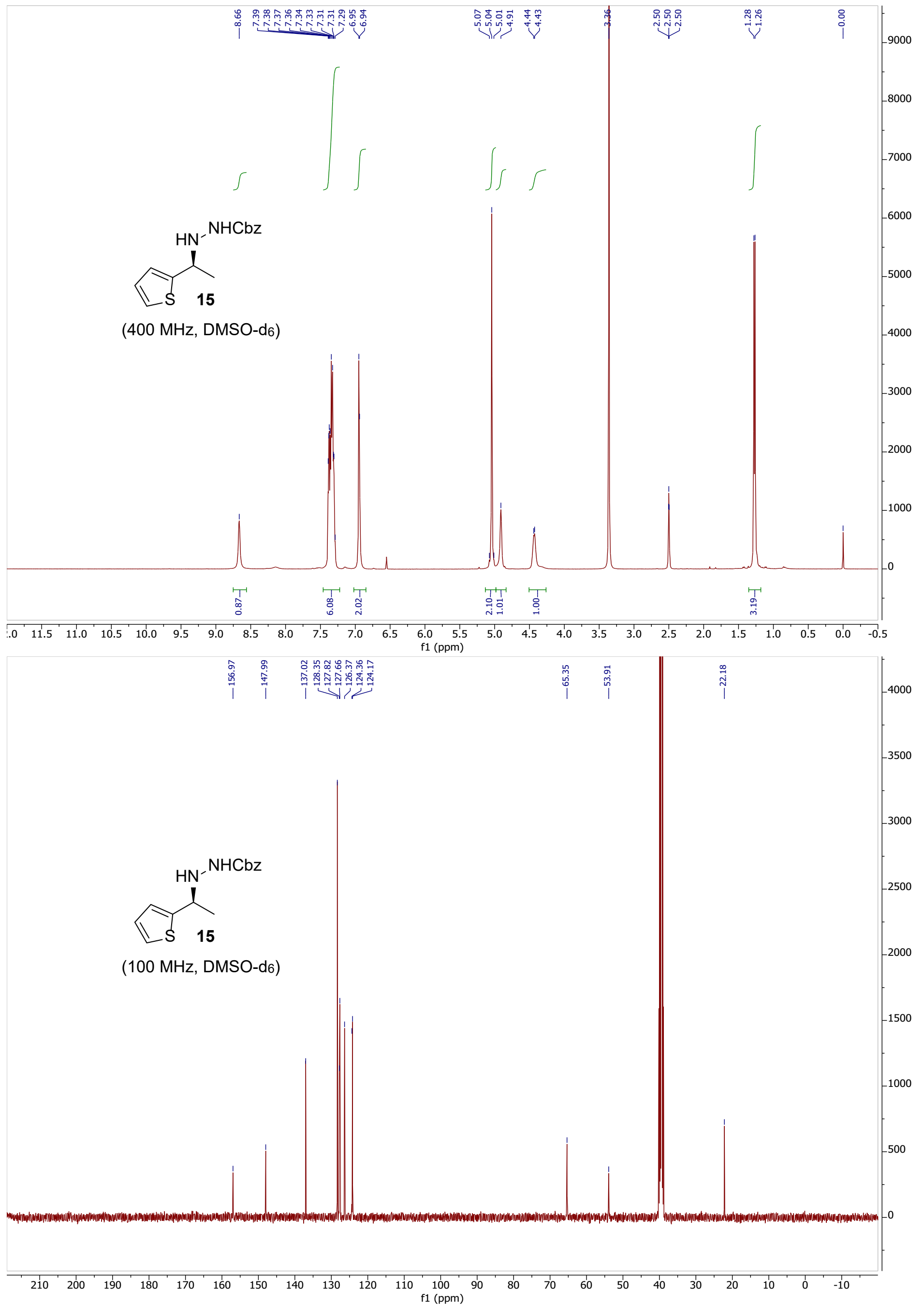




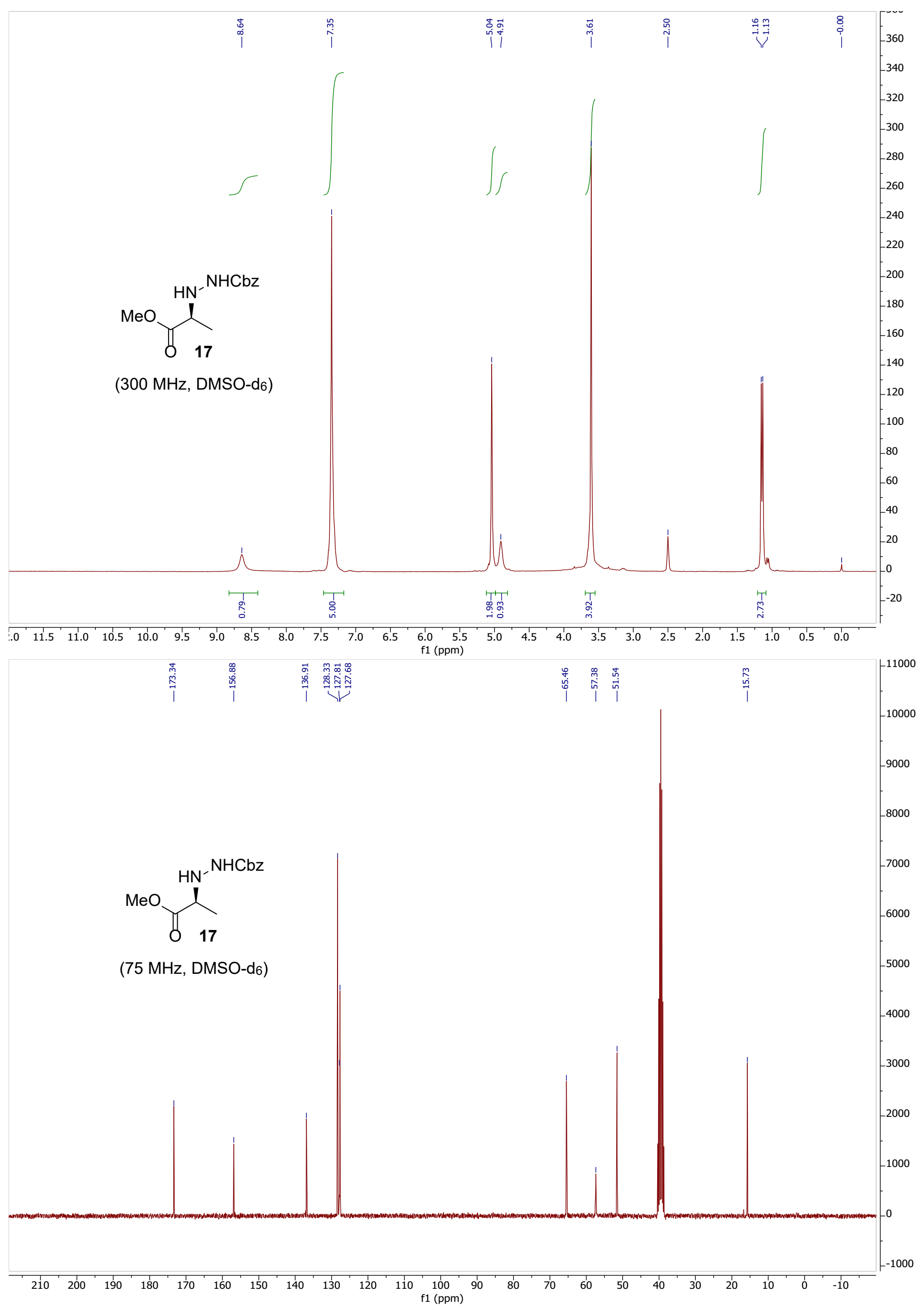


<smiles>C[C@@H](NNOC(C)(C)C)c1ccccc1</smiles>

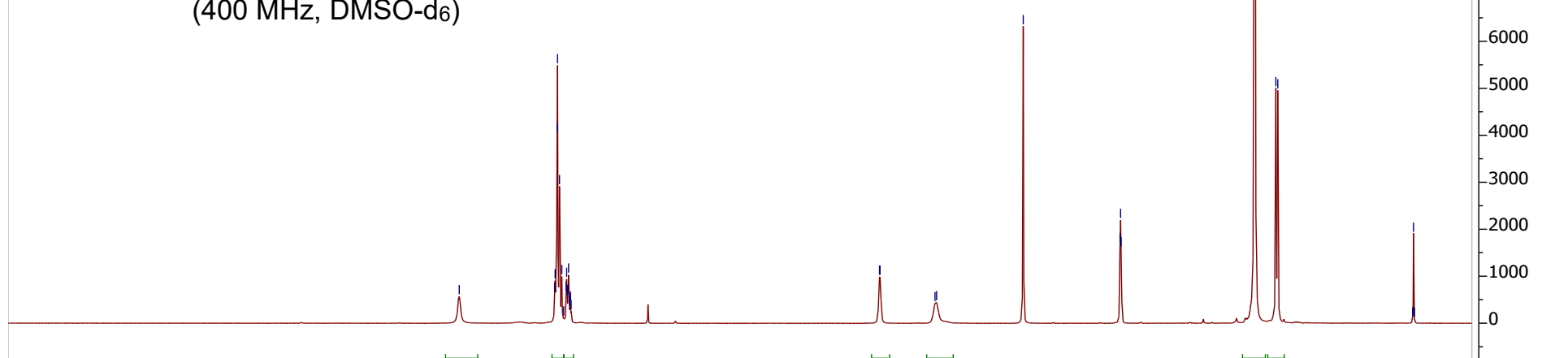

$$
\vdots 0
$$
\begin{tabular}{llllllllllll}
\hline 0 & 11.5 & 11.0 & 10.5 & 10.0 & 9.5 & 9.0 & 8.5 & 8.0 & 7.5 \\
\hline
\end{tabular} $\begin{array}{ccc}6.5 & 6.0 & 5.5 \\ & f 1(\mathrm{ppm})\end{array}$

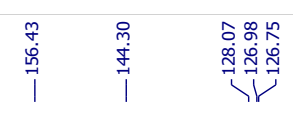

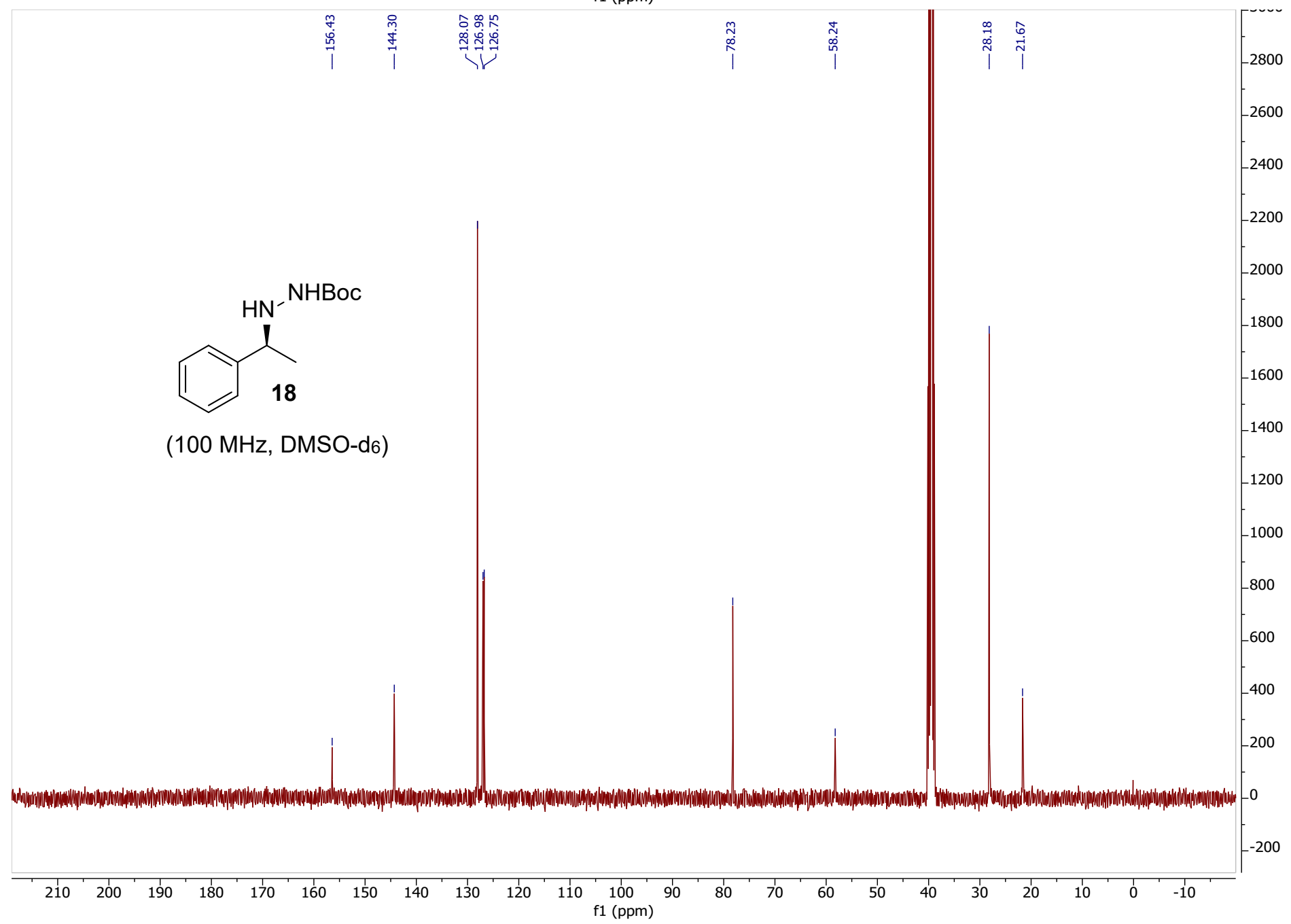

(100 MHz, DMSO-d6)

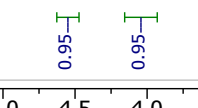




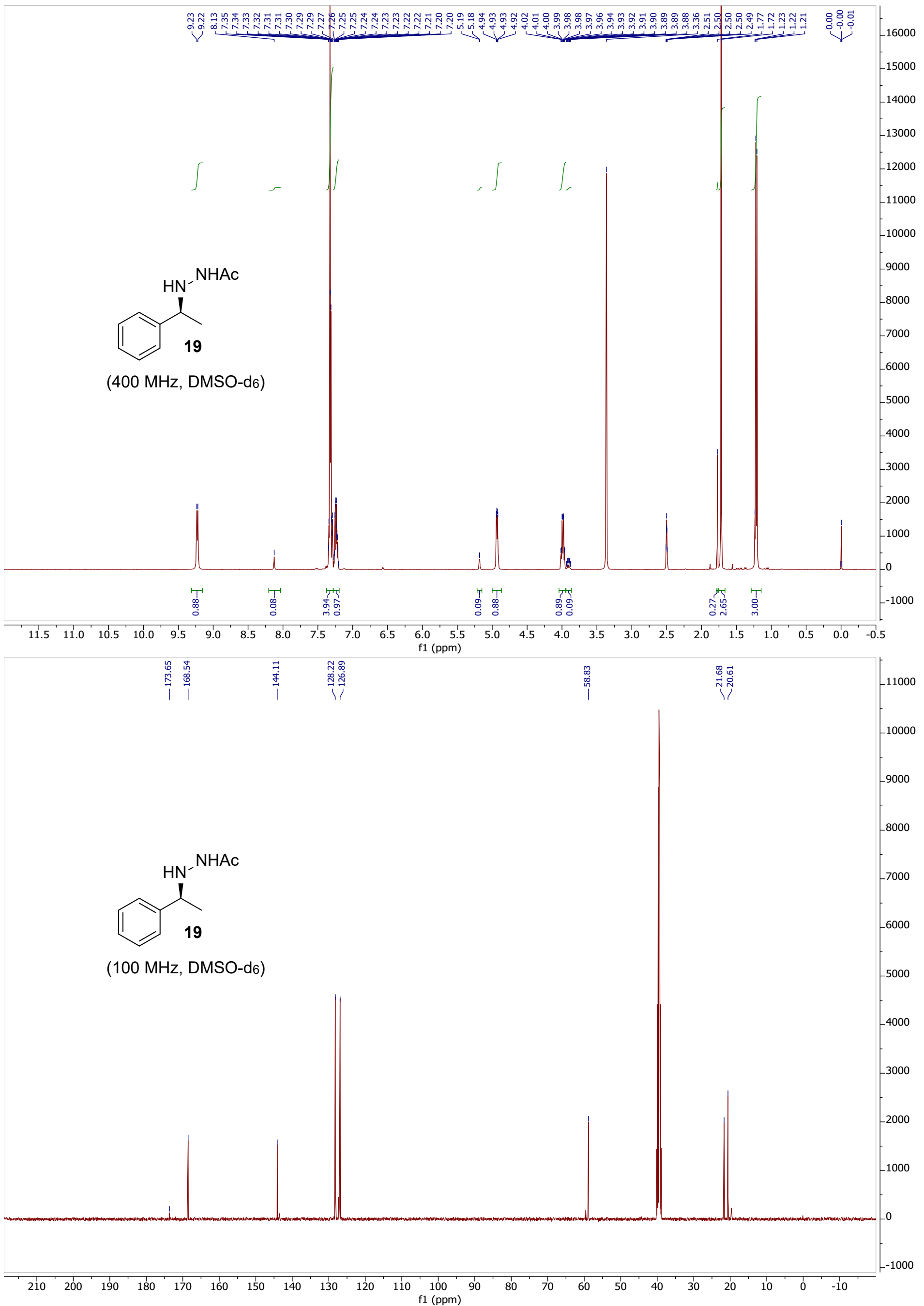




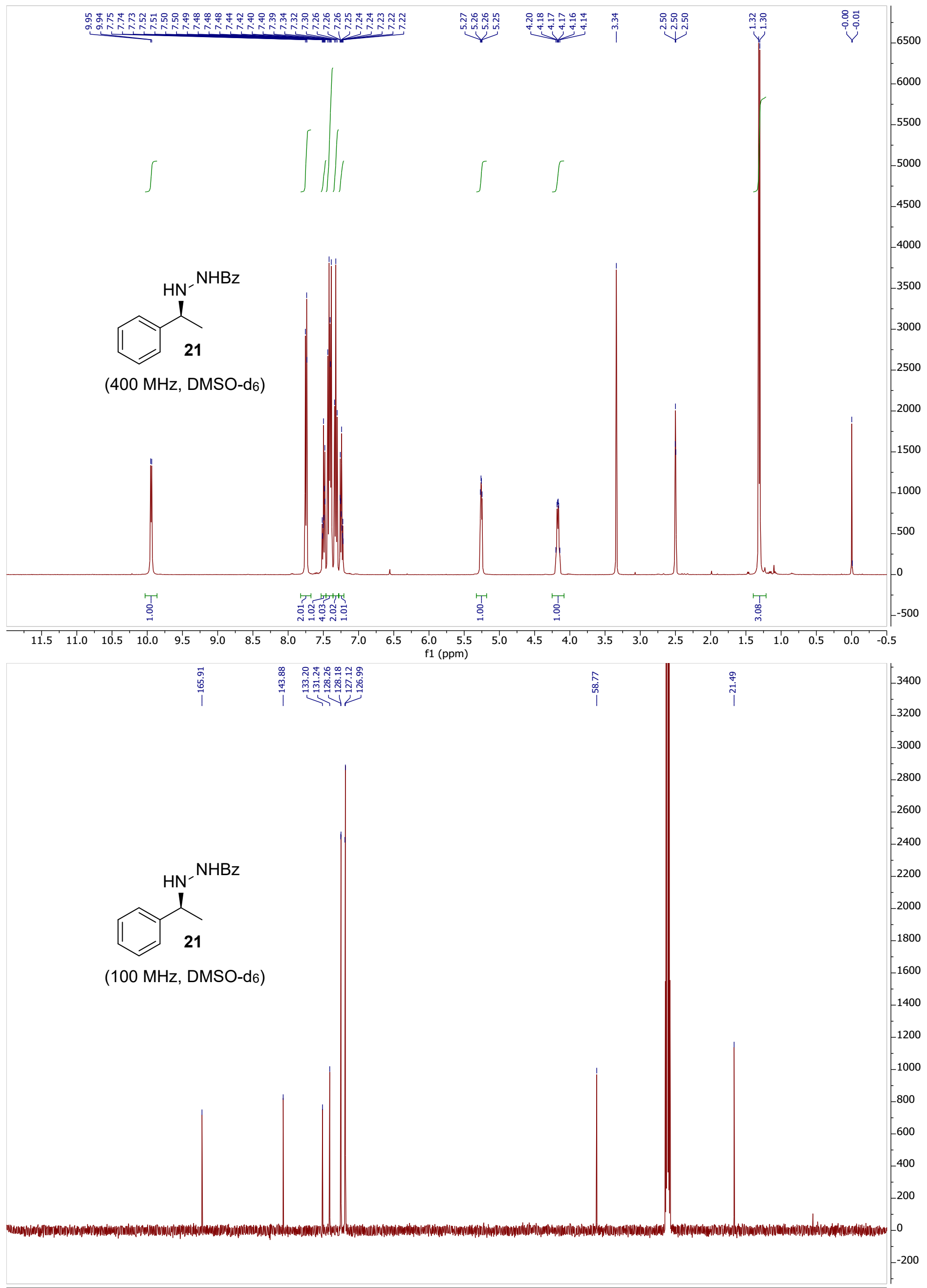




\section{References}

1) Armstrong, A.; Jones, L. H.; Knight, J. D.; Kelsey, R. D. Org. Lett. 2005, 7, 713.

2) Matunas, R.; Lai, A. J.; Lee, C. Tetrahedron 2005, 61, 6298.

3) Yoshikawa, N.; Tan, L.; McWilliams, J. C.; Ramasamy, D.; Sheppard, R. Org. Lett. 2010, 12, 276.

4) Wu, P.L.; Peng, S. Y.; Magrath, J. Synthesis 1995, 435.

5) Race, N. J.; Bower, J. F. Org. Lett. 2013, 15, 4616.

6) Zimin, D. P.; Darin, D. V.; Rassadin, V. A.; Kukushkin, V. Y. Org. Lett. 2018, 20, 4880.

7) Wang, Z.; Zhu, F.; Li, Y.; Wu, X. F. ChemCatChem 2017, 9, 94. 